\title{
A Comprehensive Examination of High-Temperature Hydrogen Attack-A Review of over a Century of Investigations
}

\author{
Kioumars Poorhaydari (D)
}

Submitted: 8 April 2021 / Revised: 30 June 2021 / Accepted: 8 July 2021 / Published online: 9 August 2021

\begin{abstract}
In this review article, many aspects of high-temperature hydrogen attack of steel are examined in a practical, topic-based format similar to that of API RP 571. This article is a result of reviewing over 220 articles on the subject, published mostly between 1938 and 2020, while some of them were already review articles citing research going back to the late nineteenth and the early twentieth centuries. The paper not only examines the historical evolution of our understanding of the damage mechanism, but it also discusses the current state of knowledge and the fields that are still under development or improvement. Questions are raised on a few important interpretations/classifications, namely the inclusion of decarburization as a form of HTHA, the division of incubation period to detectable and non-detectable stages of attack by API RP 941 and the recent distinction of non-conventional or non-classical HTHA in the literature, which may need re-evaluation. At the end, the interactions between HTHA and some other (related) damage mechanisms are discussed briefly and a failure case study is presented and discussed with respect to the use of incubation curves for the effect of an upset condition.
\end{abstract}

Keywords hydrogen attack, incubation period, non-destructive examination, Nelson curves, steel

\section{Introduction}

High-temperature hydrogen attack (HTHA or HA in short) is the permanent/irreversible deterioration of mechanical properties of steel (and some other metals/alloys) upon exposure to hydrogen at an elevated temperature. HTHA is a relatively mysterious and somewhat overlooked damage mechanism, especially when compared to other high-temperature damage mechanisms such as creep. Although HTHA was discovered in the early twentieth century (i.e., likely between 1900 and 1920), it appears that there have been periodic lapses of general knowledge and attention and, therefore, periodic re-learning of the subject among the industry end-users as well as consulting engineers or researchers in the last decades. Recently (i.e., post 2010), there has been a renewed attention to HTHA among researchers and various industry end-users dealing with steel exposed to hydrogen at elevated temperatures due to a number of factors including a catastrophic explosion in the USA in 2010 (Ref 1), increased safety concerns, subsequent revisions to guidelines and recommended practices [such as API RP 9412016 (Ref 2)] and improvements in non-destructive testing (NDT). Therefore, a need for research articles as well as review articles on HTHA has been recognized around the world to increase the knowledge about the different aspects of the damage mechanism.

Kioumars Poorhaydari, Department of Chemical and Materials Engineering, University of Alberta, Edmonton, AB, Canada. Contact e-mail: kioumars@ualberta.ca.

\begin{tabular}{|ll|}
\hline & \multicolumn{1}{c|}{ Abbreviations } \\
\hline AET & Acoustic emission testing \\
API & American Petroleum Institute \\
AUBT & Automated ultrasonic backscatter testing \\
CVN & Charpy v-notch \\
DI & Damage index \\
EDX & Energy-dispersive x-ray \\
FFS & Fitness for service \\
HA & Hydrogen attack \\
HAT & Hydrogen attack tendency \\
HAZ & Heat-affected zone \\
HE & Hydrogen embrittlement \\
HTHA & High-temperature hydrogen attack \\
IOW & Integrity operating window \\
NDE/NDT & Non-destructive examination/testing \\
$P$ & Pressure \\
$P_{\mathrm{V}}$ & HTHA parameter V \\
$P_{\mathrm{W}}$ & HTHA parameter W \\
PAUT & Phased-array UT \\
PWHT & Post-weld heat treatment \\
SEM & Scanning electron microscope/microscopy \\
$t$ & Time \\
$T$ & Temperature \\
TEM & Transmission electron microscope/microscopy \\
TFM & Total focusing method \\
TOFD & Time-of-flight diffraction \\
UT & Ultrasonic testing \\
XRD & X-ray diffraction \\
& \\
\hline
\end{tabular}

Several good review articles have been published on the subject in the last 80 years. Smialowski (Ref 3) and Feltcher and Elsea (Ref 4) separately reviewed numerous investigations on HTHA from the early 1900s to the early 1960s. Beck et al. 
(Ref 5) continued on Feltcher and Elsea's review by including research on the topic between the early 1960s and the early 1970s. Shewmon (Ref 6) published a "critical assessment" of HTHA of carbon steel and 2.25Cr-1Mo steel in 1985. In 2018, a two-part research report (practically two review articles) was prepared by the Welding Institute for UK Health and Safety Executive. Part 1 was prepared by Nageswaran (Ref 7) on the non-destructive examination (NDE) techniques related to HTHA evaluation and Part 2 was prepared by Rothwell (Ref 8 ) on the factors affecting HTHA. The twin articles covered a bibliography list of less than 50 items each (32 in Part 1 and 46 in Part 2).

This article is a result of reviewing over 220 articles published between 1938 and 2020 on the subject of HTHA, some of which were already review articles of investigations going back to the late nineteenth and the early twentieth centuries (i.e., more than a century of investigation). This article differs from the previously published articles in the format and the extent of scope. The core of the article uses a practical topic-centered format, similar to that used in API RP 571 (Ref 9), which is a familiar format for those interested in damage mechanisms and involved in failure analysis. Although the concise information provided in API RP 571 is very useful to acquire the basic knowledge about the damage mechanism, it may not be sufficient for every reader or researcher.

API RP 941 (Ref 2), on the other hand, is a dedicated publication on the subject of HTHA and the main reference for the industry. It provides the Nelson curves for material selection and risk analysis "integrity operating window" (IOW), describes the two forms of HTHA and discusses the effects of time (incubation period), stress (primary and secondary), heat treatment and cladding/overlay as well as inspection for HTHA. API RP 941-2016, however, does not discuss the effects of environmental species and corrosion, mechanisms other than methane formation, destructive examination and testing for HTHA evaluation, modification/substitutions to the Nelson curves, other prediction charts for risk assessment (particularly the HAT charts; see Section 16), modeling, or the synergistic/competitive effects of other damage mechanisms. These extra topics are covered in this article.

Although the main format of this article is topic-centered, the article starts with a section on the major historical findings as pertains to HTHA, including a table listing the major findings/events over a century in a chronological order. At the end, a brief case study that shows the use of the Nelson curves and incubation curves for carbon steel is presented. It is hoped that readers/researchers interested in a certain topic associated with HTHA will find this topic-based review a good starting point for their investigation. Clearly, no section of this article is to be considered an absolute/perfect collection of knowledge on the subject or to be used by industry to make risk-based or operational decisions.

\section{Major Findings and Historical Events}

According to Shewmon (Ref 6), HTHA was discovered in the 1920 s in Germany in ammonia synthesis plants. However, one can find earlier accounts of the identification of steel and copper embrittlement upon reaction with hydrogen at elevated temperatures at the beginning of the twentieth century by Heyn, Habor and Bosch (Ref 4, 10), also in Germany. Table 1 summarizes the important events and findings regarding HTHA in a chronological order, extracted from the literature. The table starts with information about hydrogen charging into steel and low-temperature hydrogen embrittlement and continues with the step-by-step evolution of our understanding of HTHA in terms of damage mechanism(s), development of boundary condition curves (typically referred to as the Nelson curves), effect of time and the concept of incubation period, the governing specifications, NDT, modelling, risk assessment and inspection.

Figure 1 shows the distribution of the articles published on HTHA between 1930 and 2020. It includes 320 relevant articles initially found upon a search in Compendex data base on the subject, amended by additional relevant articles found in the reference lists of the reviewed articles. The distribution, outlined by a curve, shows a short peak around 1950, a larger peak around 1965, the largest peaks around 1985 and 1996, as well as a recent peak in 2019. Such fluctuations may come from several catastrophes around the world and the subsequent increase in research as well as changes to the guidelines/ regulations, as described below.

One era of boosted research on the subject was the years prior to the World War II in Germany (Ref 14) and during and after the war in the North America (Ref 10, 37). The North American research (leading to the first peak in Fig. 1) was apparently led by Shell Development Company that resulted in the publication of the temperature-pressure boundary conditions for several steels by Nelson.

Prager (Ref 38) categorizes "post-Nelson" twentieth-century studies in the world into the following groups: several APIfunded researches in the 1960s (especially by Vitovec and leading to the second peak in Fig. 1), several studies at American universities in the 1970s and the 1980s, experimental studies on high-strength Cr-Mo steels by Materials Properties Council in the 1980 s and the 1990 s, focused studies on C0.5 Mo steel in Japan in the 1980s and the 1990s and later in the United States in the 1990s, and the theoretical studies (i.e., modeling) of void growth in Cr-Mo steels in Europe (particularly by the Dutch researchers) in the 1990s.

Plant catastrophes have been another factor in research spike, as they would call for improved safety, which in turn would require careful inspections and stringent regulations. The 1982 explosion in a desulphurization unit of Kashima Oil Refinery in Japan due to HTHA of a carbon steel pipe that resulted in five deaths and three cases of serious injury, may explain the boosted research in the 1980s in Japan (i.e., the third peak). Hasegawa (Ref 26) referred to it as "a turning point for the need to re-examine the operational conditions and materials used for plant in operation in every oil refinery" as well as developing suitable NDE methods. This eventually led to establishing the engineering parameters $P_{\mathrm{V}}$ and $P_{\mathrm{W}}$ and the hydrogen attack tendency (HAT) charts, as examined in Section 16. The subsequent removal of $0.5 \mathrm{Mo}$ steel curve from API Publication 941 in 1990 also resulted in boosted research (i.e., the fourth peak) as the change would affect many equipment already in service.

The twenty-first century studies were likely boosted by another catastrophe, i.e., the Tesoro refinery explosion in Anacortes, Washington, USA. In 2010, a deadly fire and 


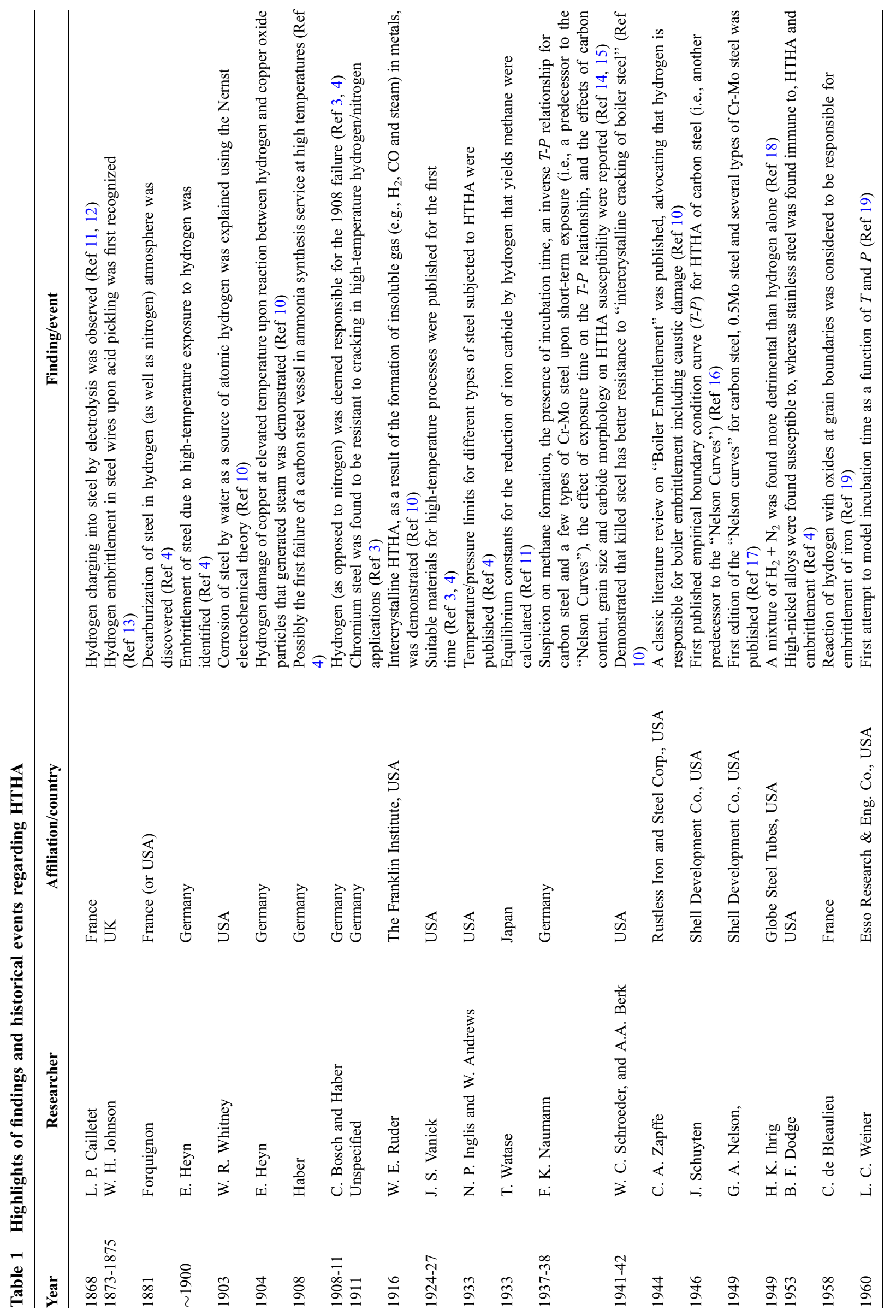




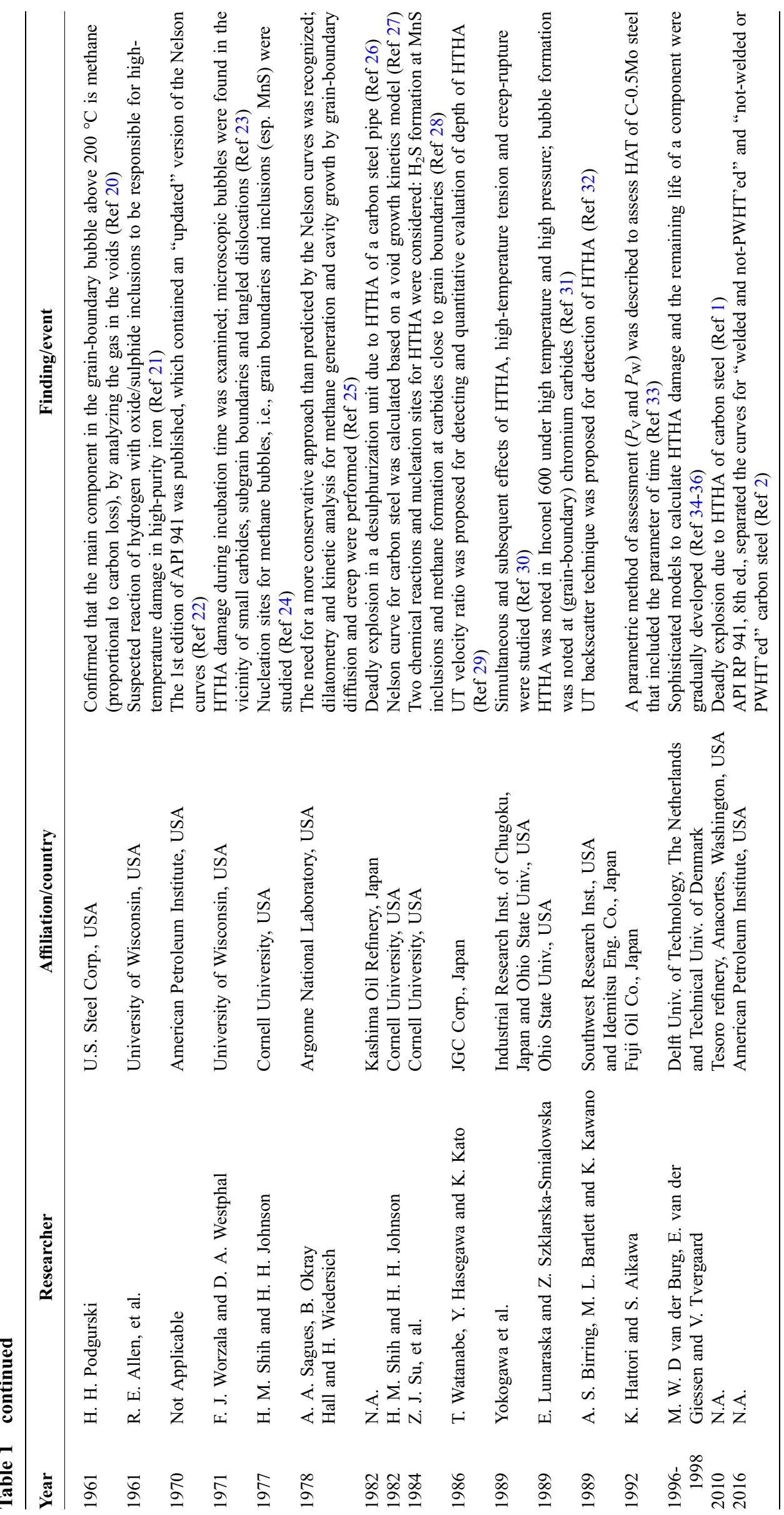




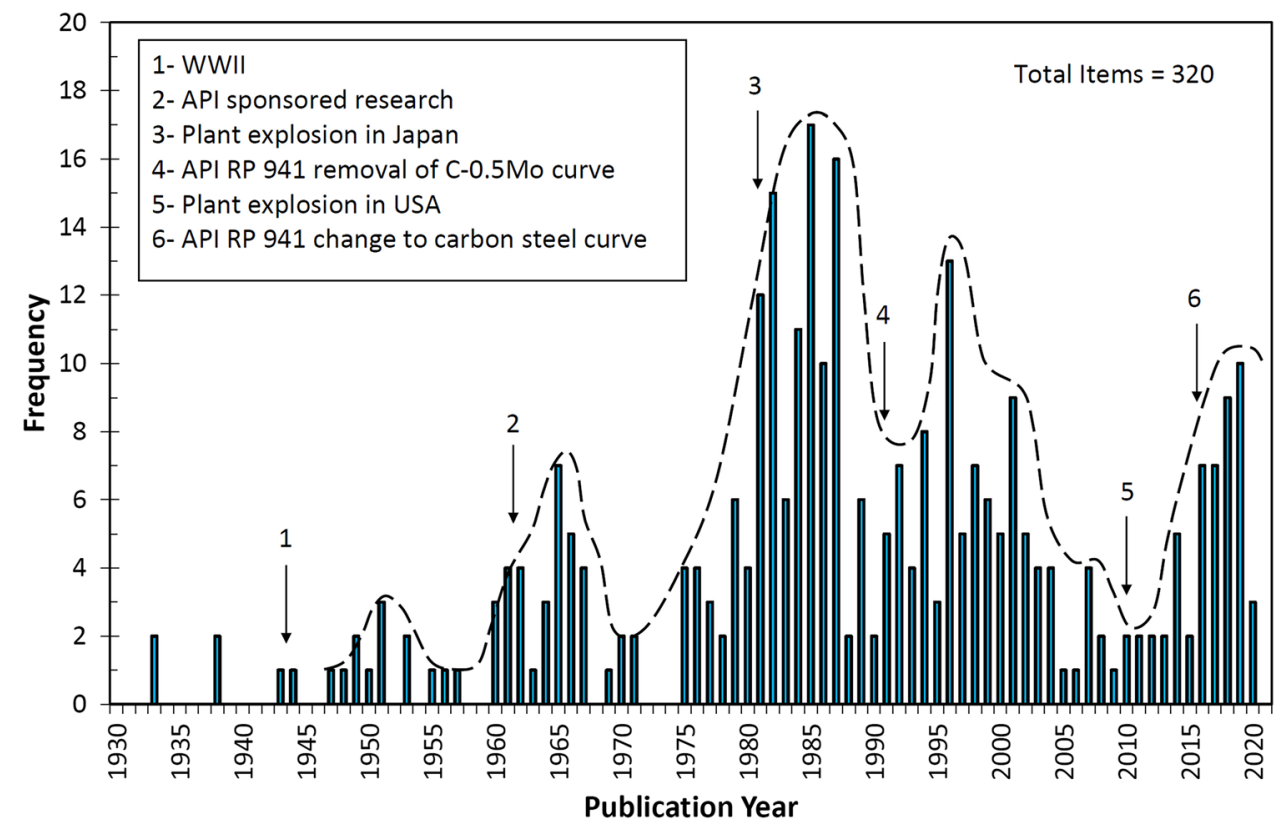

Figure 1 Publication frequency on HTHA between 1930 and 2020

explosion occurred at the refinery upon the rupture of a carbon steel heat exchanger in a naphtha hydrotreater unit that was determined to be due to HTHA. The incident occurred during the final stages of a start-up process, after the heat exchanger had been cleaned, and resulted in the immediate loss of seven workers' lives. Hydrocarbon leaks at flanges during start-ups had become a "normal" and tolerated problem. Among the subsequent investigation recommendations were banning the use of carbon steel in HTHA-susceptible service (i.e., operating above $400{ }^{\circ} \mathrm{F}\left(204{ }^{\circ} \mathrm{C}\right)$ and 50 psia $(3.5$ bar or $\left.0.345 \mathrm{MPa})\right)$ and a requirement for the use of actual operating conditions (rather than nominal) in risk/failure assessments (Ref 1). The change to the carbon steel Nelson curve in the eighth edition of API RP 941 in 2016 (Ref 2) and the safety alert on preventing HTHA (Ref 39) released also in 2016 by the United States Chemical Safety Board were for the most part consequences of this catastrophe. All these post-2010 changes led to the boosted publication as marked by the most recent peak of 2019 in Fig. 1.

\section{Description}

HTHA is defined as the permanent/irreversible deterioration of mechanical properties of a metal or an alloy upon exposure to hydrogen at an elevated temperature. HTHA is irreversible hydrogen damage (as opposed to the reversible hydrogen embrittlement at lower temperatures), since the mechanical properties cannot be recovered by a low-temperature anneal (Ref 40). The two main forms of HTHA manifestation are softening associated with surface decarburization or, more importantly, embrittlement associated with internal cavitation and fissuring along the grain boundaries. Blistering, is considered a third form of HTHA by some researchers. HTHA can affect hardness, strength, ductility and impact toughness. The source of nascent/atomic hydrogen can be a chemical reaction (specifically corrosion by water or acid, particularly when 'poisons' such as arsenic, $\mathrm{H}_{2} \mathrm{~S}$ and phosphorus are present) or thermal dissociation of molecular hydrogen (Ref 4).

\section{Terminology}

In this section, several terms related to HTHA are defined and the evolution of the terms referring to the damage mechanism is described.

\subsection{Hydrogen Damage}

This term is the broadest term that refers to mechanical property deterioration of metals by hydrogen through various forms and mechanisms. It includes (low-temperature) hydrogen embrittlement, hydrogen induced blistering/cracking, HTHA and hydride formation (in certain alloys) (Ref 41).

\subsection{Hydrogen Embrittlement (HE)}

HE refers to low-temperature (i.e., typically less than $100{ }^{\circ} \mathrm{C}$ and mostly at the ambient temperature) loss of ductility and/or cracking in metals/alloys by the actions of dissolved hydrogen in the atomic form (i.e., excluding embrittlement/damage upon reaction and combination of dissolved hydrogen atom with itself, with impurities or with metal atoms). HE covers several forms of damage that depend on material properties, manufacture and environment. It covers reversible embrittlement and cracking that are induced during manufacturing and develop prior to service as well as those that develop in service (Ref 41).

\subsection{Hydrogen Attack (HA)}

HA was the most prevalent term in the twentieth century for the irreversible high-temperature hydrogen damage, even in 
API Publication 941 (Ref 42). However, the term was also used occasionally for low-temperature $\mathrm{HE}$ in aqueous corrosive environments [e.g., (Ref 43, 44)], which was confusing.

\subsection{High-Temperature, High-Pressure Hydrogen Attack}

This was a transitional term between HA and HTHA that was used [e.g., (Ref 45)] to exclude the low-temperature HE.

\subsection{Hot Hydrogen Attack (HHA)}

Another transitional term that was used towards the end of the twentieth century [e.g., (Ref 38,46$)$ ] to exclude the lowtemperature HE.

\subsection{High-Temperature Hydrogen Attack}

HTHA has been the most prevalent term in the twenty-first century for the irreversible high-temperature hydrogen damage. Reference to the full term can be traced back in the 1990s [e.g., (Ref 47)] and the full acronym appears in the $5^{\text {th }}$ edition of API RP 941 in 1997, although other transitional acronyms such as HHA (Ref 48) were also used in the 1990s. The term HTHA (rather than HA) is used in this report as the main title/acronym for the damage for consistency.

\section{API Publications (Standards and Recom- mended Practices)}

\subsection{API RP 941}

This is a Recommended Practice on "Steels for Hydrogen Service at Elevated Temperature and Pressure in Petroleum Refineries and Petrochemical Plants." It was first published in 1970 and has received several revisions since then. The current edition is the eighth, released in 2016. Although its main objective is to establish safe operating limits (temperature and pressure) for carbon and alloy steels in services susceptible to HTHA, it discusses many aspects of HTHA, such as the effects of time, heat treatment, stress, composition and cladding. API RP 941 is the main reference publication for HTHA in industry.

\subsection{API TR 941-A}

Formerly appearing as API TR 941, this is "The Technical Basis Document for API RP 941." It discusses the plant and laboratory sources of the data in the Nelson curves, along with several other aspects of HTHA.

\subsection{API RP 581}

This is a Recommended Practice for "Risk-Based Inspection Methodology" applicable to fixed equipment in petrochemical industries. The first edition released in 2000 addressed HTHA technical module as an appendix. The current edition (i.e., third, April 2016) consists of three parts: "Inspection Planning Methodology" as Part 1, "Probability of Failure Methodology" as Part 2 and "Consequence of Failure Methodology" as Part 3. Section 19 of Part 2 presents a simple "conservative screening criterion" to determine the degree of susceptibility to HTHA (e.g., high vs. low) of the components in service. It provides a guideline for determining a damage factor for HTHA, based on the generic steel type, temperature and hydrogen partial pressure.

\subsection{API RP 584}

This is a Recommended Practice on "Integrity Operating Windows", first published in 2014 (i.e., the current edition). It is a guide for establishing an IOW program to avoid unexpected critical damage (in general) in refining and petrochemical industries. Some examples of IOW's applicable to HTHA in hydro-processing are provided in a figure (i.e., Fig. 5 in API RP 584).

\subsection{API RP 579-1}

This is a Recommended Practice on "Fitness for Service", first released in 2000 as API RP 579. The current edition (i.e., third, released in 2016) does not yet address HTHA. However, it has been reported (Ref 49) that a "Part 15" is under development by a joint industry project to address assessment techniques for HTHA in a "future edition" of API 579-1.

\subsection{API RP 571}

This is a Recommended Practice on "Damage Mechanisms Affecting Fixed Equipment in Refining Industry", first released in 2003. The current edition (i.e., third) was released in 2020 and, similar to the previous editions, it covers many aspects of HTHA concisely.

\section{Affected Industries}

The affected industries include those dealing with hydrogenation or dehydrogenation processes, ammonia production, oil refining, coal conversion (i.e., liquefaction or gasification), methanol synthesis and boiler tubes (Ref 9, 17).

\section{Affected Materials}

Carbon steel (along with $0.5 \mathrm{Mo}$ steel) is the most susceptible material. Low-alloy steels and some medium-alloy and high-alloy steels are also susceptible. Austenitic stainless steels are the least susceptible (Ref 4). Nevertheless, some bubble formation at the carbides is reported in the austenitic stainless steels (Ref 50, 51). Some non-ferrous alloys, such as nickel alloys (e.g., K-Monel, Inconel and Hastalloy B) under high temperature and high hydrogen pressure (Ref $4,31,52)$ and copper alloys (Ref 10), are also susceptible.

\section{Mechanisms}

It is suspected that different mechanisms may be involved based on material type, chemical composition, microstructure and temperature of exposure. All these mechanisms involve a chemical reaction between atomic hydrogen and a solute, a particle or an inclusion in the material. The most recognized mechanism involves a chemical reaction between hydrogen and carbon in solution in steel (or carbides in the microstructure) to form methane $\left(\mathrm{CH}_{4}\right)$. Consumption of carbon in the matrix will promote dissolution of carbides (esp. cementite) to keep the atomic carbon in solution in balance (Ref 53). Since the methane molecules are too large to diffuse out of the steel, they 
cause significant pressure buildup that results in the formation of cavities or voids (also referred to as methane bubbles) at the grain boundaries or other areas of high interfacial energy (e.g., inclusion surfaces). Fissures typically form upon the growth (via creep) and linkage of methane bubbles. "The formation and coalescence of methane bubbles into grain-boundary fissures and ultimately cracks" is referred to as HTHA "incipient attack" (Ref 54).

Note that hydrogen molecules can also be present in the cavities, as proven by gas chromatography or mass spectrometry by many researchers (Ref 55-57). However, their size and pressure is typically much less than those of methane. In theory, methane pressure can vary from being comparable to that of the hydrogen pressure to two orders of magnitude higher, depending on the carbide stability (Ref 58). Even ethane formation in the bubbles (along with methane and hydrogen) has been reported (Ref 50). A methane pressure of up to $2500 \mathrm{MPa}$ inside the bubbles was calculated by Odette and Vagarali (Ref 59) for carbon steel.

Hydrogen may also interact with other impurities (e.g., O, N and $\mathrm{S}$ ) or inclusions and produce insoluble gasses (such as water vapor, $\mathrm{NH}_{3}$ and $\mathrm{H}_{2} \mathrm{~S}$ ) that may or may not result in cracking (Ref 4, 10, 28). A classic example of the reaction between hydrogen and unstable oxides at the grain boundaries to produce steam is the hydrogen damage of copper at an elevated temperature, which is referred to as "copper sickness". This reaction can result in diametrical growth and internal fissuring. Deoxidization of copper with boron or ferrosilicon significantly improves resistance to hydrogen attack (Ref 10). Silver is susceptible to a similar damage mechanism as copper (Ref 6).

\section{Stages of Attack}

\subsection{Incubation Period}

During the incubation time after exposure to hydrogen, no detectable damage (by means of mechanical testing) occurs. However, very small bubbles (about $100 \mathrm{~nm}$ in average size, i.e., too small to affect the mechanical properties or to be observed by optical microscopy) were observed by electron microscopy in the vicinity of small carbides during the incubation stage (Ref 23). Such bubble formation results in volume change that is detectable by dilatometry (Ref 60). In fact, using a highly sensitive dilatometer (with a sensitivity of less than $10^{-6} \Delta L / L$ ), Sundararajan and Shewmon (Ref 61) showed that swelling initiates at a constant rate without any significant delay (i.e., $\Delta L / L=k t$ ) upon exposure of carbon steel to hydrogen at $400{ }^{\circ} \mathrm{C}$ and $21 \mathrm{MPa} \mathrm{H}_{2}$.

Incubation period is typically determined by the initiation of a property change and as such it depends on the sensitivity of the test. The higher sensitivity of impact toughness to HTHA damage initiation (e.g., compared to carbon concentration or tension test) was demonstrated early in the twentieth century by Naumann (Ref 14, 15).

\subsection{Main Classifications}

Different researchers have recognized different stages/steps of attack, whether in terms of number or description. The main categorization is based on the change in mechanical properties. Inglis and Andrews (Ref 4) separated the stages of the attack based on the ability to recover mechanical properties. The first stage of attack was the absorption of hydrogen and the resulting reversible embrittlement that could be mitigated by a lowtemperature anneal (i.e., hydrogen venting). The "later stages" were referred to as decarburization and fissuring, which would result in irreversible embrittlement of steel (i.e., loss of ductility and strength). Similarly, Ciuffreda and Rowland (Ref 62) considered three stages of attack in 1957, namely (1) hydrogen diffusion into the steel (reversible loss in ductility), (2) decarburization (irreversible softening) and (3) intergranular fissuring (irreversible embrittlement).

Weiner (Ref 19) produced experimental curves of HTHA damage percent versus time of exposure for carbon steel at different temperatures and hydrogen pressures and recognized four stages of attack: (1) incubation (with no detectable deterioration of mechanical property), (2) rapid attack (with steep rate of deterioration), (3) transition (with a decreasing rate of deterioration) and (4) steady state (with a low rate of deterioration). These four stages, also echoed by Worzala and Westphal (Ref 23), are re-plotted in Fig. 2. Weiner observed that with an increase in temperature or pressure the curve is shifted to shorter times (i.e., a reduction in the incubation period) without a significant change in the shape of the curve.

However, most researchers after that [such as Vitovec (Ref 63) and Vagarali and Odette (Ref 64)] recognized only the three main stages of attack by neglecting the transition part: (1) incubation, (2) rapid attack and (3) steady state or saturation (i.e., properties reaching the final value asymptotically upon carbon exhaustion). The sixth edition of API RP 941 (2004) also presented these three clear stages of attack. However, the seventh edition of API RP 941 (2008) divides the incubation time into two stages, one undetectable by advanced NDE and one "detectable by advanced NDE techniques and no noticeable change in properties is detectable by mechanical testing". The eighth edition of API RP 941 (2016) also recognized the four stages described in the previous edition but changed the second stage to "damage is detectable optically, possibly detectable by advanced NDE, and mechanical properties are partially deteriorated." Another change in the eighth edition was adding a statement to the fourth stage (i.e., the final stage), reading "carbon in solution is reduced to compromise material mechanical properties to a level where cracking can occur." There are several issues with these changes that may require reevaluation of the statements in API RP 941. First, the descriptions and divisions are of mixed criteria (i.e., lab-based mechanical property change, inspection by NDE, and failure in service). Second, detection of any microscopic damage in the incubation period (i.e., prior to grain-boundary crack formation) by advanced NDE is highly unlikely at present (see Section 12). Third, even the observation of microstructural evidence of attack by optical microscopy occurs after material has suffered from HTHA in terms of mechanical property deterioration (Ref 62) and therefore it cannot be achieved during the incubation period by definition (the observation may be achievable at a transitional stage between incubation and rapid attack). Lastly, cracking occurs in the rapid attack stage and not the final stage (i.e., saturation upon carbon consumption).

\subsection{Other Classifications}

There are also some other classifications of the stages of attack in the literature. These are typically based on more 


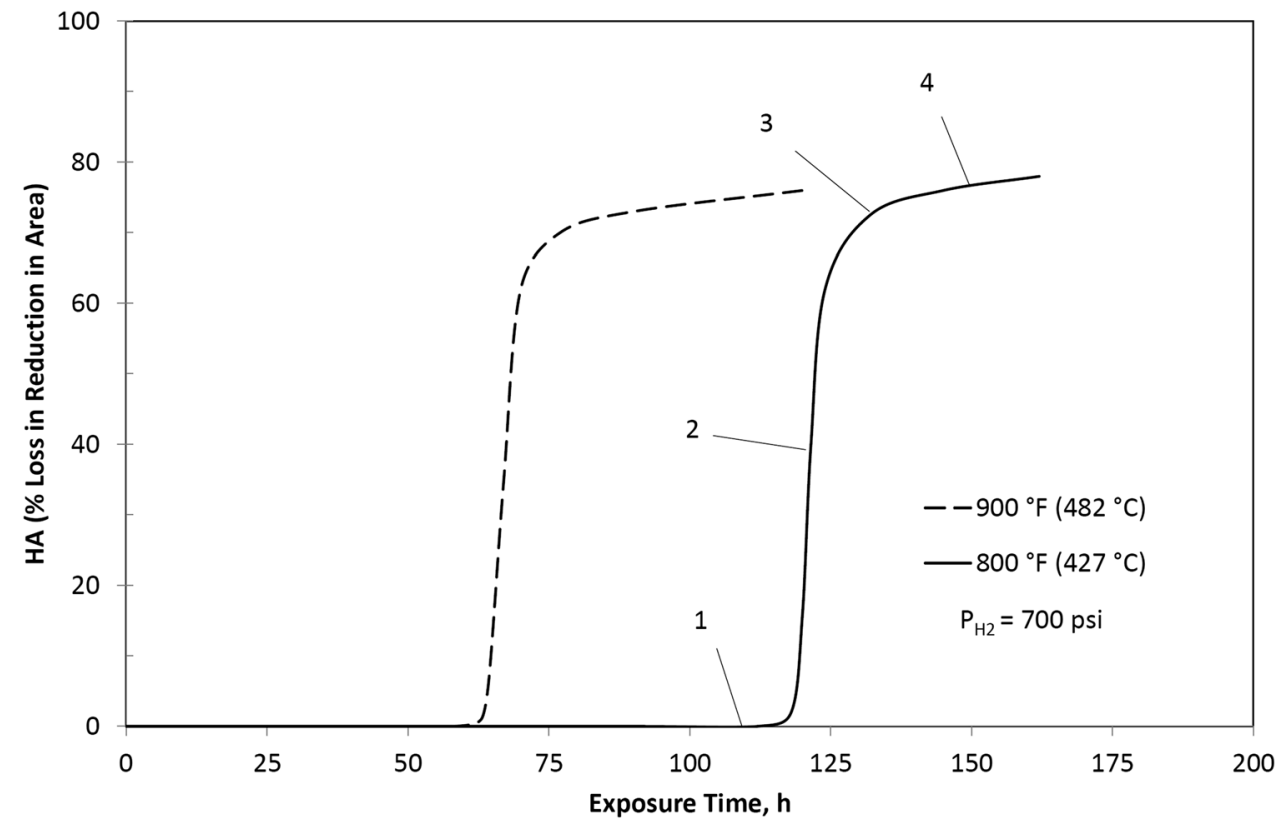

Figure 2 Examples of hydrogen attack curves for carbon steel as a function of time after Weiner (Ref 19), showing four stages of attack (redrawn from the original graph)

specific mechanisms or reactions rather than mechanical property changes. For instance, Vitovec considered five "stages of hydrogen attack of steel" (which could be better termed as stages of cavity growth) in Ref 63: (1) methane pressure buildup inside the microscopic voids, (2) cavity growth by vacancy diffusion, (3) cavity growth by dislocation creep, (4) decrease in fissure growth rate and (5) partial void shrinkage.

In 1982, Vitovec recognized eight steps for the entire hydrogen and carbon chemo-physical reactions (Ref 65): (1) hydrogen molecule adsorption and dissociation at the metal surface, (2) dissolution of dissociated atoms into steel, (3) diffusion of atomic hydrogen to the reaction site, (4) dissolution of metal carbide into metal and carbon atoms in solution, (5) diffusion of carbon to the reaction site, (6) formation of $\mathrm{H}_{2}$ at the site, (7) adsorption of carbon at the site and (8) formation of methane molecule upon reaction between $\mathrm{H}_{2}$ and $\mathrm{C}$. Clearly, in this classification, Vitovec was interested in kinetic analysis and determining the rate controlling step of reaction rather than the general mechanical property change.

Another classification is provided by al-Arada and al-Otaibi (Ref 66): (1) methane formation associated with decarburization, (2) microcrack formation at grain boundaries upon methane pressure build-up and (3) microcrack propagation leading to failure (i.e., macrocrack formation). This classification is not based on mechanical property change or percent of hydrogen attack either, but based on damage evolution in a sample.

It should be realized that the stages of attack as applied to (small) laboratory samples do not apply to actual equipment in service. The equipment in service is typically exposed to hydrogen from the inside surface only. As such, there will be a significant damage degree gradient across the wall of the container, which brings out both an advantage and a disadvantage. The disadvantage is that damage becomes difficult to detect (affecting a fraction of sample that is not easily accessible). The advantage is that the inside surface might have suffered an advanced stage of attack (e.g., fissuring or microcrack formation that is likely detectable by advanced
NDE), without the general state of the container becoming critical, i.e., there should be enough time to remove the equipment from service before a catastrophic failure happens, as long as a proper inspection program and control of operating conditions are in place.

\section{Appearance and Morphology}

HTHA is an internal damage mechanism and does not show any wall loss or bulk deformation [except for some swelling (Ref 25)]. The two main types of damage recognized in various literature (including API RP 941) are surface decarburization and internal decarburization/fissuring. Surface decarburization occurs under the combination of relatively low hydrogen pressure and high temperature, whereas internal decarburization and fissuring occurs under the combination of relatively high hydrogen pressure and low temperature (Ref 2, 53). In surface decarburization, it is the carbon that (due to the increased temperature) can diffuse to the surface to form carbonaceous gasses such as methane and $\mathrm{CO} / \mathrm{CO}_{2}$. In internal decarburization, atomic hydrogen penetrates into the steel and reacts with carbon in solution. As a result, the produced gas is entrapped internally and can cause cracking. Micro-fissures are typically found along the grain boundaries within the wall close to the inside surface of a vessel. In the case of carbon steel, they form at the interface between a pearlite colony and the ferrite matrix or between adjacent ferrite grains (Fig. 3a). Internal decarburization with fissuring is the main type of HTHA damage, as it is typically this form (rather than surface decarburization) that can cause serious damage to the steel, mostly hard to detect and leading to catastrophic failures. In fact, the inclusion of surface decarburization as a form of HTHA may be questionable. Surface decarburization is certainly a related damage mechanism and has been marked as an upper shelf in the Nelson curves (Section 16.1.1). 

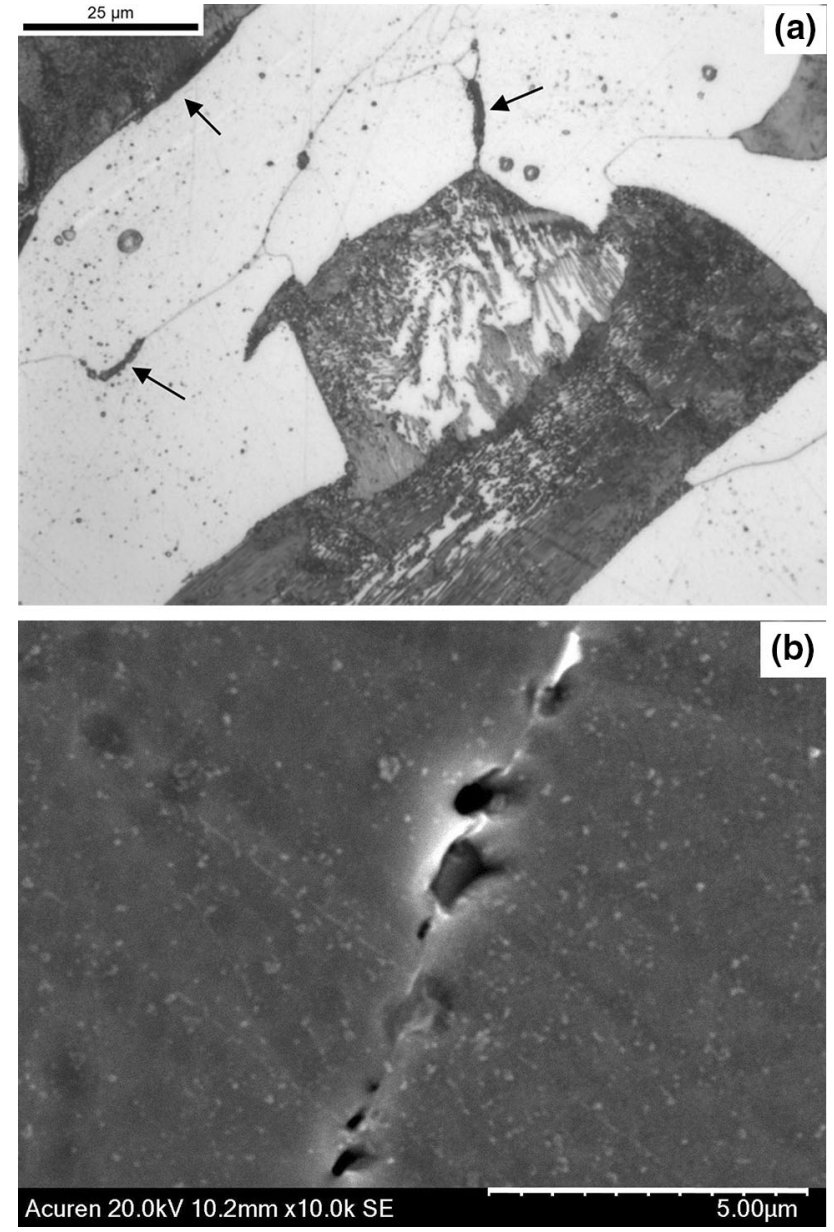

Figure 3 HTHA internal damage in carbon steel: (a) optical image depicting pearlite decomposition and grain boundary fissuring at a magnification of $1000 \times$; (b) SEM image showing grain-boundary bubbles at a magnification of $10,000 \times$

In the internal damage, fissures can form as a result of the linkage of individual methane bubbles/voids along grain boundaries (Fig. 3b), i.e., in a microscopically ductile manner through diffusion and creep deformation. Conceivably, there can be some fissure formation through a microscopically brittle (or less ductile) manner, e.g., upon decohesion of the grain boundary interface after some pressure buildup at the interface and promoted by external stress, impurities, etc. Final fractures, promoted by the initial HTHA damage, may occur as a result of an overload, creep cracking (i.e., a synergy between HTHA and creep), fatigue or corrosion-fatigue cracking, or low-temperature hydrogen embrittlement upon shutdowns. In equipment under significant external stress, it is quite conceivable that the initial HTHA damage facilitating the failure is overlooked, when the secondary damage mechanism becomes the predominant one. Sometimes HTHA damage is manifested only by reduction of mechanical properties without significant microstructural alteration or cracking. Part of such damage could be from HE (See Section 19). Note that bubbles and micro-fissuring can form along grain boundaries without significant pearlite decomposition, as documented through detailed laboratory examinations by Sagues et al. (Ref 25).

Some researchers recognize blistering as a third form of HTHA (Ref 21). Blistering occurs by accumulation of molec- ular gasses (mostly hydrogen and methane) along planar interfaces in the microstructure parallel and close to the inside surface. In general, blistering is irreversible damage and can occur by hydrogen damage in relatively soft materials, i.e., carbon steel and C-0.5Mo steel, or by HTHA (Ref 67).

Recently, there has also been a distinction for non-conventional (Ref 68) or non-classical (Ref 69) HTHA damage. McLaughlin et al. (Ref 68) reported a case of "intergranular hydrogen-assisted cracking similar to HTHA" in a hydrogenerator carbon steel piping with welds that had no post-weld heat treatment (PWHT). The hydrogen attack had occurred over a short period of time ("unlike conventional HTHA") upon a sudden increase of hydrogen partial pressure over three years. They did not observe grain-boundary methane bubbles and made an argument that cracking susceptibility depended on partial pressure and not temperature. They concluded that the observed intergranular cracking was different from typical/conventional HTHA cracks that initiate as grain-boundary methane bubbles and are driven by a time-dependent creep mechanism.

Silfies et al. (Ref 69) discussed the "non-classical" HTHA damage morphology reported on non-PWHT'ed carbon steel piping. They distinguished between "surface decarburization and more 'typical' HTHA damage" on the one hand and cracks near welds or on hot-bent elbows without bulk or surface decarburization on the other.

It is not clear if either of the above-mentioned recent distinctions (i.e., non-conventional or non-classical HTHA), which have been echoed by some other researchers afterwards [e.g., (Ref 70)], is necessary, as they appear to be based on some variations of damage characteristics and likely driven by various operational or material conditions. A factor that can affect the crack morphology and is difficult to assess on piping is external stress due to bending. Poorhaydari (Ref 71) reported a case of HTHA cracking in the sub-critical heat-affected zone (HAZ) of a flange without significant internal/bulk decarburization or pearlite decomposition. A factor in the failure was bending stress that resulted in cracking on one side of the flange. It was reported as early as in 1933 that under a high level of external stress, HTHA fissures could form ahead of internal decarburization ( Ref 4).

\section{Factors}

Several factors affect HTHA susceptibility and the rate of attack. These factors, namely temperature, pressure, time, stress, microstructure, composition, microstructure and environment, are discussed in this section.

\subsection{Temperature}

- There seems to be a limiting temperature for any system for the onset of the attack [generally considered above $200{ }^{\circ} \mathrm{C}$ for carbon steel (Ref 24, 25)]. The limiting temperature depends on other factors such as material composition, pressure, stress and exposure time.

- As pointed out by Fletcher and Elsea (Ref 4), Nelson reported "apparent permissible service temperatures", for any range of hydrogen pressure up to $14 \mathrm{ksi}(9.7 \mathrm{MPa})$, to be approximately $220^{\circ} \mathrm{C}\left(430^{\circ} \mathrm{F}\right)$ for carbon steel, 
$330{ }^{\circ} \mathrm{C} \quad\left(620^{\circ} \mathrm{F}\right)$ for $\mathrm{C}-0.5 \mathrm{Mo}$ and $1 \mathrm{Cr}-0.5 \mathrm{Mo}$ steel, $400{ }^{\circ} \mathrm{C}\left(750{ }^{\circ} \mathrm{F}\right)$ for $2 \mathrm{Cr}-0.5 \mathrm{Mo}$ steel, $540{ }^{\circ} \mathrm{C}\left(1000{ }^{\circ} \mathrm{F}\right)$ for $3 \mathrm{Cr}-0.5 \mathrm{Mo}$ steel and $610{ }^{\circ} \mathrm{C}\left(1125^{\circ} \mathrm{F}\right)$ for $6 \mathrm{Cr}-0.5 \mathrm{Mo}$ steel. These limits were the horizontal legs of the Nelson curves (Ref 72).

- The damage becomes faster and more severe with increasing temperature (up to a certain temperature, as determined by the thermodynamics of the chemical reaction). HTHA is more sensitive to temperature than the other factors. For example, it was shown that a difference of $25^{\circ} \mathrm{C}$ could change the situation from not attacked to severely attacked, while doubling pressure from 1000 to $2000 \mathrm{~atm}$ resulted in a moderate increase in damage (Ref 4). Prager (Ref 38) reports that "generally" the rupture life in hydrogen drops in half with an increase of $25^{\circ} \mathrm{F}\left(\sim 14{ }^{\circ} \mathrm{C}\right)$.

- It is reported that the methane formation reaction (between hydrogen and cementite) is thermodynamically possible only below $600{ }^{\circ} \mathrm{C}\left(1110^{\circ} \mathrm{F}\right)$, after which the free energy change of the reaction becomes positive (Ref 23). Therefore, an HTHA susceptibility temperature range of approximately $200-600{ }^{\circ} \mathrm{C}$ (with an $\mathrm{H}_{2}$ pressure of $0.7 \mathrm{MPa}$ minimum) is typically considered (Ref 73). As such, API RP 581 (Ref 74) stipulates a conservative temperature of $177^{\circ} \mathrm{C}\left(350^{\circ} \mathrm{F}\right)$ for "screening" of the susceptible steels for HTHA (provided the pressure is above $0.345 \mathrm{MPa}$ or 50 psia).

- Above approximately $600{ }^{\circ} \mathrm{C}\left(1100{ }^{\circ} \mathrm{F}\right)$, the rate of carbon diffusion increases so much that decarburization occurs mostly at the surface. This, if continued, can result in complete decarburization without fissuring (Ref 16, 53).

\subsection{Pressure}

- There also seems to be a limiting hydrogen partial pressure for any system for the onset of the attack. The pressure depends on other factors such as material composition, temperature, stress and exposure time.

- Pressure and temperature are the two main parameters used traditionally to assess the susceptibility of steel to HTHA through "the Nelson Curves" (see Section 16).

- The damage becomes faster and more severe with increasing pressure, although HTHA is not as sensitive to pressure as it is to temperature. Prager (Ref 38) reports that the rupture life in hydrogen is a function of the reciprocal of pressure squared, meaning that the life drops approximately four times when hydrogen pressure doubles. This is in support of the theoretical assumption that hydrogen partial pressure and methane pressure have a quadratic relationship: $P_{\mathrm{CH} 4}=P_{\mathrm{H} 2}{ }^{2} / \mathrm{K}$ (Ref 11,16$)$ (K is the equilibrium constant of the reaction).

- Different researchers have considered different values for minimum hydrogen pressure required for HTHA in carbon steel, such as $0.7 \mathrm{MPa}$ (Ref 25), $1 \mathrm{MPa}(\operatorname{Ref} 6), 2 \mathrm{MPa}$ (Ref 75), $2.5 \mathrm{MPa}$ (Ref 76) and $3 \mathrm{MPa}$ (Ref 77). The $0.7 \mathrm{MPa}$ limit (equivalent to $6.9 \mathrm{~atm}$ or $100 \mathrm{psia}$ ) is consistent with the vertical leg of the Nelson curve for nonwelded (or welded and PWHT'ed) carbon steel according to API RP 941-2016 ( $\operatorname{Ref} 2$ ). The limit would be half of that (i.e., $0.345 \mathrm{MPa}$ or $50 \mathrm{psia}$ ) for welded (but not PWHT'ed) carbon steel, which now coincides with the limit stipulated by API RP 581-2016 (Ref 74) for "screening" of steel components for HTHA (provided the temperature is above $177^{\circ} \mathrm{C}$ or $350{ }^{\circ} \mathrm{F}$ ).

\subsection{Exposure Time}

- The pair of limiting temperature and pressure decreases by increasing exposure time. This was first reported by Naumann (Ref 4,14). Several contours of $T-P$ curves can be established for each material at different exposure times.

- No systematic work on the effect of exposure time on the $T-P$ limits was done for several decades as pointed out in a review report by Ciuffreda and Rowland (Ref 4 ). Weiner produced graphs of HTHA (\%) versus exposure time at several temperatures and pressures in 1960, as explained in Section 9 and shown in Fig. 2.

- Later, Nelson provided supplementary "incubation period curves" for carbon steel and C-0.5Mo steel in 1965 that showed the effect of time on $T-P$ curves between the safe and unsafe zones (Ref 78). The incubation curves appear in API RP 941-2016 for non-welded (or welded and PWHT'ed) carbon steel. However, such curves are not available for other materials or conditions (i.e., Cr-Mo steel or welded but not PWHT'ed carbon steel). The curves can be used to determine if an excursion above the safe operating conditions can result in an HTHA damage for a given period of time ( Ref 2).

- Since 2013, Engineering Equity Group has been working on a mechanistic model of HTHA damage propagation and has established multiple time-dependent "Prager curves" for carbon steel and C- $0.5 \mathrm{Mo}$ steel (instead of Nelson curve for each) for a rapid screening "safe zone" assessment as well as prediction of the failure time for a pair of pressure and temperature (Ref 49). (See Section 16.2.)

\subsection{Stress}

- Although pre-existing external or internal/residual stress is not necessary for HTHA, stress is a very important parameter that promotes HTHA and lowers the temperature for attack significantly (Ref 79). Stress is not addressed directly in the Nelson curves, although it is inherent to the empirical industrial results.

- As cited by Fletcher and Elsea (Ref 4), Inglis and Andrews reported in 1930s that, under high stress and moderate temperature, fissuring could happen ahead of decarburization upon HTHA. In the absence of stress, decarburization could occur without any fissuring (under certain conditions).

- Sakai and Asami (Ref 80) observed that the void growth rate in a quenched-and-tempered $2.25 \mathrm{Cr}-1 \mathrm{Mo}$ steel subjected to $200 \mathrm{kgf} / \mathrm{cm}^{2}$ (2845 psi or $19.6 \mathrm{MPa}$ ) hydrogen at 525 and $550{ }^{\circ} \mathrm{C}$ increased 3-4 times upon application of $10-20 \mathrm{kgf} / \mathrm{cm}^{2}$ (1-2 MPa) external stress.

- Yokogawa et al. (Ref 30) noted that the HTHA incubation 
time vanished with an applied stress and decarburization occurred faster in specimens under stress. Residual stress, which typically increases with increasing material strength, also promotes HTHA.

- Through a parametric approach, Sakai et al. (Ref 81) estimated that an applied stress of $150 \mathrm{MPa}(21.8 \mathrm{ksi})$ is equivalent to increasing the hydrogen pressure by approximately $5 \mathrm{MPa}$ for a $2.25 \mathrm{Cr}-1 \mathrm{Mo}$ steel at $850{ }^{\circ} \mathrm{F}\left(454{ }^{\circ} \mathrm{C}\right)$ and would lower the critical temperature for HTHA by approximately $50^{\circ} \mathrm{F}\left(28^{\circ} \mathrm{C}\right)$ at a hydrogen pressure of $10 \mathrm{MPa}$ or approximately $25^{\circ} \mathrm{F}\left(14^{\circ} \mathrm{C}\right)$ at a hydrogen pressure of $20 \mathrm{MPa}$.

- Stresses associated with thermal gradients (as experienced during equipment cool down or start-up, for instance) can promote HTHA, even in the equipment operating in the "safe zone" of the Nelson curves. Therefore, the frequency of thermal gradient events (such as unplanned shutdowns and subsequent start-ups) can be a factor in the formation of HTHA (Ref 82).

- HTHA may occur at "high-stress" areas, as found in nonPWHT'ed welds (Ref 1) and at stress risers (Ref 62, 71).

\subsection{Material Composition}

- HTHA is dependent on carbon availability. Therefore, it depends on the solubility limit of carbon in the matrix and the stability of the carbides at the elevated temperature. Cementite in carbon steel is not a very stable carbide at the elevated temperature of service (over $200{ }^{\circ} \mathrm{C}$ ) and dissolves relatively easily upon interaction with atomic hydrogen. As such, carbon steel and C-0.5Mo steel are the most susceptible materials to HTHA.

- It was found by Naumann (Ref 14) that alloying with strong carbide formers such as tungsten, zirconium, chromium, molybdenum, vanadium, titanium and niobium increases the thermal stability of the carbides and make the material more resistant to HTHA. With increasing chromium content, carbides evolve from $\mathrm{M}_{3} \mathrm{C}$ to $\mathrm{M}_{7} \mathrm{C}_{3}$ to $\mathrm{M}_{23} \mathrm{C}_{6}$ (Ref 83). Some metals can make types $\mathrm{M}_{2} \mathrm{C}$ and $\mathrm{M}_{6} \mathrm{C}$ carbides. In $2.25 \mathrm{Cr}-1 \mathrm{Mo}$ steels, all these five types can be present, which in the order of increasing stability are $\mathrm{M}_{3} \mathrm{C}, \mathrm{M}_{2} \mathrm{C}, \mathrm{M}_{7} \mathrm{C}_{3}, \mathrm{M}_{23} \mathrm{C}_{6}$ and $\mathrm{M}_{6} \mathrm{C}$ (Ref 84).

- The formation and relative stability of carbides may depend on the other elements in the steel, such as silicon (Ref 85). Imanaka et al. (Ref 86) showed that reduction of silicon to $0.15 \mathrm{wt} . \%$ maximum and addition of $0.15 \mathrm{wt} . \%$ vanadium maximum improves HTHA resistance of $2.25 \mathrm{Cr}-1 \mathrm{Mo}$ steel, likely due to an increase in carbide stability (Ref 87 ). Addition of $0.26 \mathrm{wt} . \% \mathrm{Si}$ increased bubble density (by a factor of 1.3) and diameter (by a factor of 1.4), compared to the steel with $0.016 \mathrm{wt} . \%$ Si (Ref 88). Imanaka et al. (Ref 89) concluded that reducing sulphur, nitrogen and silicon and increasing vanadium will improve HTHA resistivity of Cr-Mo steels, whereas niobium addition is ineffective in terms of HTHA resistivity. Other examples in the literature of HTHA resistance improvement upon composition modifications that could change carbide composition, type or morphology/distribution include additions of $\mathrm{V}-\mathrm{Nb}$ to $2.25 \mathrm{Cr}-1 \mathrm{Mo}$ steel (Ref 90, 91), $\mathrm{V}$-Ti-B to $3 \mathrm{Cr}-1 \mathrm{Mo}$ steel (Ref 92 ), $\mathrm{V}-\mathrm{Nb}-\mathrm{B}$ to $2.25 \mathrm{Cr}$ -
$1 \mathrm{Mo}$ steel and vanadium to $9 \mathrm{Cr}-1 \mathrm{Mo}$ steel (i.e., making Grade 91) (Ref 93). Many investigations have shown that vanadium has a more pronounced effect in improving HTHA resistance of Cr-Mo steels than $\mathrm{Ti}$ or $\mathrm{Nb}$ [e.g., (Ref 94)]. Note that the austenitization temperature for quench-and-temper heat treatment of steel should increase (according to the type of carbide makers added) in order to take advantage of such additions, as undissolved coarse carbides (and nitrides) left in the material after low-temperature austenitization may limit the beneficial effect of such carbide makers (Ref 95, 96).

- Even small amounts of carbide stabilizing elements, such as $\mathrm{Cr}$ and $\mathrm{Mo}$ in carbon steel coming from remelted scrap alloys, can improve the HTHA resistance. This led Nelson to publish an additional "safe operating" chart and an inset on the main chart in 1965 for a range of Mo from 0 to $0.5 \%$ (Ref 97).

- High-strength microalloyed steels (e.g., with 0.02-0.14\% $\mathrm{Nb}$ ) are typically more resistant to HTHA than carbon steel, and in some cases more resistant than C-0.5 Mo, due to the formation of $\mathrm{MC}$ type carbides that are more stable than $\mathrm{M}_{3} \mathrm{C}$ carbides (Ref 61).

- Manganese was found to have limited effect, while nickel and silicon were found to be ineffective in improving resistance to HTHA (Ref 3,18$)$.

- Increase of carbon content from 0.05 to $0.17 \%$ in a 2.25Cr-1Mo steel resulted in a linear decrease in HTHA incubation time (Ref 98).

- Very-low-carbon steels are also susceptible to HTHA. Yokogawa et al. (Ref 30) showed that carbon in solution in ferrite was responsible for HTHA in a single-phase $0.011 \% \mathrm{C}$ steel at $9.9 \mathrm{MPa}$ hydrogen and $500{ }^{\circ} \mathrm{C}$.

- Pishko et al. (Ref 99) investigated the effect of steelmaking on the early stage of HTHA of carbon steel (i.e., formation of fine bubbles along grain boundaries). Four types of steel, namely silicon-killed, aluminum-killed, rare-earth-metal-treated and electroslag-refined, were investigated at $450{ }^{\circ} \mathrm{C}$ and $6.5 \mathrm{MPa}$ hydrogen as well as $275^{\circ} \mathrm{C}$ and 7.6 MPa hydrogen. They found that steelmaking practice had a significant effect on the incubation time; attack occurred fastest in rare-earth-metal-treated steel and slowest in Al-killed steel.

- Although as a general statement, it has been reported that higher impurity levels (and larger grains) make the grain boundaries more prone to HTHA cracking (Ref 68), the actual effect of impurities on HTHA resistance appears to be complicated and not completely/clearly determined. Rosenthal et al. (Ref 100) reported that impurities such as $\mathrm{P}$ and $\mathrm{S}$, which would segregate at grain boundaries, can have a retarding effect in grain-boundary fissuring by HTHA in carbon steel. They suspected that the retarding effect was due to the reduction of grain-boundary energy and reduction of grain-boundary diffusion. Sakai et al. (Ref 88, 94, 101, 102) investigated the effects of the impurity elements phosphorous, tin, arsenic and antimony on HTHA bubble formation and growth in Cr-Mo steels. They found that an addition of $0.016 \mathrm{wt} \% \mathrm{Sn}$ or 0.016 wt.\% $\mathrm{Sb}$ increased the bubble density (by a factor of $2-3$ ) in a $2.25 \mathrm{Cr}-1 \mathrm{Mo}$ steel, whereas an addition of 0.016 wt. $\%$ P or $0.017 \%$ As decreased the bubble density (by a factor of 0.5-0.75). On the other hand, using an impurity factor $\mathrm{X}=(10 \mathrm{P}+5 \mathrm{Sb}+4 \mathrm{Sn}+\mathrm{As}) \times 100, \mathrm{Ki}-$ 
mura et al. (Ref 103) found no effect of impurity levels on HTHA resistance of a C-0.5Mo steel.

- Some elements may not affect the carbide stability significantly, but they can still improve the HTHA resistance by increasing the creep strength of the material (since creep deformation is involved in HTHA bubble growth). Molybdenum was considered to have such an effect (Ref 104).

- Shida et al. (Ref 105) reported that addition of $10 \mathrm{ppm}$ boron to Cr-Mo steels compromised the HTHA resistance of simulated HAZ significantly. However, in a 1987 paper by the same group of researchers (Ref 106), it is reported that the combined addition of $0.002-0.090 \% \mathrm{Ti}$ and 8 $94 \mathrm{ppm}$ B improved the HTHA resistance of Cr-Mo steels. These additions affected the carbide type (e.g., $\mathrm{M}_{7} \mathrm{C}_{3}$ without boron and $\mathrm{M}_{23} \mathrm{C}_{6}$ with boron), reportedly.

- Stainless steel is not immune to HTHA and may show some decarburization upon long-term exposure to hydrogen at elevated temperature (e.g., $566{ }^{\circ} \mathrm{C}$ and $4.4 \mathrm{MPa}$ $\mathrm{H}_{2}$ ) (Ref 107). Bubble formation at the carbides were also reported for a Type 321 stainless steel exposed to $14 \mathrm{MPa}$ $\mathrm{H}_{2}$ at $600{ }^{\circ} \mathrm{C}$ for $480 \mathrm{~h}$ (Ref 50).

\subsection{Microstructure and Prior Treatments}

- It was found decades ago that (unlike in creep damage) larger grains are more susceptible to HTHA (Ref 4).

- Spheroidizing treatment can increase the incubation period of HTHA attack of a carbon steel five times by coarsening the carbides and reducing carbon in solution (Ref 19). Lopez (Ref 108) reported significant improvement in HTHA resistance (as determined by dilatometric swelling rate measurements as well as tension testing) of rare-earthmetal-treated quenched-and-tempered carbon steel upon a spheroidization heat treatment, whereas such treatment did not affect the resistance of electroslag-refined steel.

- Tempering temperature and time affect the type and stability of carbides in low-alloy steels, which in turn affect the HTHA susceptibility. Su et al. (Ref 109) reported that precipitates in a $1.25 \mathrm{Cr}-0.5 \mathrm{Mo}$ changed from predominantly $\mathrm{M}_{3} \mathrm{C}$ type to predominantly $\mathrm{M}_{23} \mathrm{C}_{6}$ type with increasing tempering temperature from 520 to $710{ }^{\circ} \mathrm{C}$. However, the effect of tempering temperature on HTHA resistance was not linear. The resistance was lowest at $620{ }^{\circ} \mathrm{C}$, which was attributed to the instability of $\mathrm{M}_{3} \mathrm{C}$ precipitates at the grain boundaries. It should be noted that the tempering time was only $30 \mathrm{~min}$ in their experiments, which is generally considered low for stabilizing the carbides.

- Thermal cycle of welding can alter the grain size, decrease the stability of carbides (e.g., cementite) and result in residual stress development. As such, welded components are typically more susceptible to HTHA, particularly in the HAZ. PWHT enhances HTHA resistance. It is known that if proper PWHT is not carried out, the HTHA resistivity of an alloy steel (e.g., C-0.5Mo or Cr-Mo steels) can be as low as that of carbon steel (Ref 110). A proper heat treatment for $\mathrm{C}-0.5 \mathrm{Mo}$ steel after normalizing or after welding can consist of tempering at $650{ }^{\circ} \mathrm{C}$ minimum for $5 \mathrm{~h}$ minimum (Ref 111,112 ). Very high tempering tem- peratures (e.g., $690{ }^{\circ} \mathrm{C}$ for $16 \mathrm{~h}$ ) are also recommended for PWHT of Cr-Mo steel weldments (Ref 113).

- It is not clear (or cannot be determined) which weld/HAZ sub-region is most susceptible to HTHA. Parthasarathy and Shewmon (Ref 114) showed through dilatometric study coupled with microstructural examination of bubble density that the weld metal of a $2.25 \mathrm{Cr}-1 \mathrm{Mo}$ steel was more affected by HTHA than the HAZ and the base metal was the least affected. Prager (Ref 38) observed that, in general, HAZ was more susceptible to HTHA than weld metal and the HAZ sub-region of failure (i.e., fine-grained vs. coarsegrained) varied among the tests and $T-P$ conditions. Schlogl and Van der Giessen (Ref 115) used modeling and numerical analysis and found that the weld regions of a $2.25 \mathrm{Cr}-$ 1 Mo steel in the order of decreasing accumulated damage are fine-grained HAZ, coarse-grained HAZ, weld metal and base metal. The main factor in the difference was methane pressure variation in these regions. Manna et al. (Ref 116) showed through some laboratory tests on Cr-Mo steels that the most susceptible sub-region would depend on the level of applied stress; in the absence of stress, weld metal, finegrained HAZ and inter-critical HAZ exhibited the highest degree of cavitation, whereas in the presence of stress the most damaged regions (exhibiting microcracks) were base metal and inter-critical HAZ, while weld metal was least damaged. It is clear that not only many factors (such as stress, composition and thermal history) affect the area of highest susceptibility, the criterion for HTHA degree of damage (particularly those concerning bubble formation in the incubation period vs. mechanical property deterioration that is more pronounced in the rapid attack stage) can also affect the conclusions. It should be added that there have been several reports of failure of piping weldments just outside the HAZ (Ref 68, 71, 117, 118), i.e., the sub-critical HAZ, that has not been studied well either experimentally or by modelling.

- Prior cold work can accelerate HTHA and reduce the incubation time ( $\operatorname{Ref} 4,21,119)$.

- Researchers have reported bubble nucleation in the vicinity of carbides and pearlite colonies (Ref 23). It was observed that continuous carbide precipitation along grain boundaries would increase the susceptibility of carbon steel to HTHA, compared to when carbides were dispersed in the matrix homogeneously (Ref 75). On the other hand, Shih and Johnson (Ref 24) noted MnS inclusions to be associated with bubbles in carbon steel subjected to HTHA, indicating that inclusions are preferred sites for bubble nucleation. Su et al. (Ref 28) considered that this might be due to $\mathrm{H}_{2} \mathrm{~S}$ formation rather than methane formation, whereas Allen et al. (Ref 53) concluded that carbon is needed for cavitation/fissuring at inclusions based on the observation that the inclusions near pearlite colonies were more affected and fissuring was accompanied by local decarburization. Allen et al. also concluded that reduction of inclusions may not be a solution for HTHA mitigation (Ref 53). Bubble formation at second phases (such as MnS inclusions) in addition to grain boundaries have been reported by many researchers [e.g., (Ref 79)].

- Preferred bubble and fissure nucleation along manganese sulphide and manganese silicate inclusions in Si-killed 
steel exposed to HTHA at $450{ }^{\circ} \mathrm{C}$ and $6.5 \mathrm{MPa} \mathrm{H}_{2}$ for 44-220 h was observed by Ransick and Shewmon (Ref 120 ). Upon $15 \%$ cold work, fissuring along grain boundaries was promoted.

- Prior exposure to neutron diffraction can cause formation of helium bubbles along grain boundaries (1-100 nm in size) at $T>0.5 T_{\mathrm{m}}$ as well as reduction of the incubation time for HTHA at lower temperatures (Ref 73).

\subsection{Environmental Species}

- It has been reported that moisture enhances decarburization (Ref 4, 121).

- Damage is high at extreme ends of $\mathrm{pH}$ and is the lowest around the neutral point $(6.8-7.2)$. Dissolved $\mathrm{H}_{2} \mathrm{~S}$ (acidic condition) and cyanide (alkaline condition) were considered to be very detrimental (Ref 72). Antimony, cyanide and hydrogen sulphide, if present, can increase the rate of hydrogen diffusion into the steel as they act as poisons for hydrogen recombination at the surface, where corrosion occurs.

- In mixed hydrogen-nitrogen environments and under $T-P$ conditions conducive to HTHA, carbon steel loses carbon and absorbs nitrogen. Formation of a nitride case on austenitic stainless steel, which shows the highest resistance to HTHA, has been reported (Ref 18 ). In ammonia synthesis gas, a very thin and brittle nitride case forms above $545^{\circ} \mathrm{C}\left(850^{\circ} \mathrm{F}\right)$ on steels with over $2 \% \mathrm{Cr}$ (Ref 37,122$)$. Wagner et al. (Ref 123) also reported a case of compromised HTHA resistance of $2.25 \mathrm{Cr}-1 \mathrm{Mo}$ steel after 8 years of service in an ammonia plant at $400{ }^{\circ} \mathrm{C}$ due to nitriding and decarburization at the inside surface and suspected transformation of otherwise stable carbides to carbonitrides. They referred to the damage induced by the ammonia-bearing synthesis gas as chemically induced hydrogen cracking.

- A small addition (10 vol.\%) of $\mathrm{H}_{2} \mathrm{~S}$ to $\mathrm{H}_{2}$ was reported to inhibit HTHA of carbon steel at $525^{\circ} \mathrm{C}\left(977{ }^{\circ} \mathrm{F}\right)$ after $240 \mathrm{~h}$ of exposure by the formation of a sulphide scale at the surface (Ref 124). This is while testing in $\mathrm{H}_{2}$ (without $\mathrm{H}_{2} \mathrm{~S}$ addition) resulted in significant tensile strength reduction and formation of fissures. It should be noted that the test specimens did not have any mill scale (or oxide scale) that could provide some protection to hydrogen ingress and it is not known if the results are still valid for a longterm exposure.

- Fouling and deposit formation in boiler tubes can result in under-deposit corrosion and hydrogen ingress into the steel. Cases of decarburization and intergranular fissuring were reported close to the inside surface of waterwall tubes in a high-pressure boiler as a result of HTHA promoted by poor boiler conditions (Ref 125).

- A combination of oxygen and chlorides in boiler feedwater used in a steam-assisted recovery oil extraction operation was suspected to promote acidic conditions and internal corrosion in steam lines that resulted in HTHA decarburization and fissuring under the corroded areas and subsequent rupture of the tubes (Ref 126).

\section{Inspection and Evaluation}

\subsection{Non-destructive Examination}

Visual inspection would not be applicable as HTHA exhibits no macroscopic evidence (except for blistering in some cases). NDE/NDT methods, particularly different types of ultrasonic testing (UT), have been tried with various degrees of success (mostly limited, but under continuous improvement) in detecting various stages of HTHA damage. Stage 1 HTHA or "incubation period" involves the formation of small microscopic damage (i.e., mostly grain-boundary cavities) that is generally not detectable by NDE (Ref 2). It is only in the later stages of the attack (i.e., Stages 2 to 4 as per API 941 classification or Stages 2 and 3 as per general mechanical property change classification, when grain-boundary fissures, microcracks or blisters, and macrocracks form) that damage may be detected by some NDE techniques. In general, welds add to the challenge in detecting HTHA damage, as they are a source of signal "interruption" due to the associated reinforcement (as well as root bead geometry/anomalies), misalignment and/or coarse-grained HAZ (Ref 33).

API RP 941:2016 discusses the suitability of various NDE techniques for HTHA assessment and the Welding Institute Research Report RR1134 (Ref 7) provides a critical review of all the available NDE techniques for HTHA inspection. The reportedly most successful types of UT are backscatter, velocity ratio, time-of-flight diffraction (TOFD) and UT spectrum analysis (Ref 54, 66, 127). HTHA damage decreases ultrasonic wave velocity and increases its attenuation and backscatter (Ref 32). Manual or automated UT (using longitudinal or shear wave techniques) are not capable of detecting early stages of HTHA fissuring. UT attenuation method can be useful, when there is significant damage for example in the form of blisters at the inside surface or fissuring over a significant fraction of wall thickness (Ref 128). Ohtsuka and Shindoh (Ref 129) used a "pulse echo digital overlap" method to improve UT velocity and attenuation results by eliminating errors and poor display that originated from chattering of an oscilloscope cathode ray tube display. Nomura and Imanaka (Ref 130) reported that variation in attenuation with a change in signal frequency had high potential for detecting HTHA damage at an early stage of attack after the incubation time. Automated ultrasonic backscatter testing (AUBT) is considered by some researchers to be the most effective NDE method for HTHA assessment (Ref 7). It involves backscatter technique along with spectral analysis and velocity-ratio measurement. Kawano et al. (Ref 131) reported a very high level of sensitivity for backscatter UT technique (to the extent of detecting bubbles at the inner part of the wall) and introduced an "equivalent defect size" as a UT measure of HTHA damage degree. McGovern et al. (Ref 132) proposed a nonlinear UT method of wave mixing for through-wall detection of HTHA damage from the outside surface of vessels. They claim that their nonlinear methods (with or without pulse inversion) can detect HTHA damage at an earlier stage than linear UT (e.g., backscatter) could (Ref 133).

On the other hand, some researchers in the USA have found unsatisfactory results with the API RP 941 recognized UT methods, despite more satisfactory results obtained in Asia and Europe. Nugent et al. (Ref 134) concluded that velocity ratio, amplitude and spatial averaging UT methods were not reliable or suitable for HTHA detection. Conventional shear wave, high frequency shear wave and angle beam spectral analysis were 
found unreliable too. Attenuation, spectral analysis and frequency dependence were found somewhat successful. In their 2018 article, Nugent et al. (Ref 135) concluded that TOFD and phased-array UT (PAUT) with or without total focusing method (TFM) provide the best results in evaluating HTHA. They provided procedural details on how to run these methods successfully for detecting and sizing HTHA damage. Neve et al. (Ref 136) also reported more satisfactory results with PAUT, TOFD and TFM than AUBT, particularly in terms of the detection limit.

From all these accounts, it appears that the degree of success of a method/technique is not merely an inherent factor and it depends largely on the procedural details (such as selection of parameters) and the skill of the technician who executes the test. Birring et al. (Ref 137) pointed out the subjectivity in some UT interpretations and emphasized on the importance of proper technique, proper procedure and inspector skill. They also provided some insight on how to distinguish between UT signals from hydrogen damage cracks and stringers of inclusions (that can be a source of miscall). Yamani and Deriche (Ref 138) used an "automated ultrasonic detection and classification system" to minimize human errors and emphasized on creating a reliable database and "advanced signal processing techniques" for correct identification of defects.

UT can measure the portion of the wall thickness affected by HTHA (i.e., the depth of attack), as long as at least $10-20 \%$ of the wall thickness contain fissures and/or blisters. This measurement can be used as "lost wall" in the assessment of fitness for service (FFS) (Ref 127). Watanabe et al. (Ref 29) proposed a UT velocity ratio technique to assess the depth of attack. They found a range of traveling time ratio between the longitudinal and shear waves $\left(t_{\mathrm{L}} / t_{\mathrm{S}}\right)$ of $0.546-0.588$ for different degrees of attack on components in service and proposed a critical value of 0.550 to be used as a criterion for existence of significant HTHA damage. Takatsubo and Yamamoto (Ref 139), on the other hand, used a "pore response function" of ultrasound waves for quantitative evaluation of area fraction and size of microcracks in hydrogen attacked steel.

Acoustic emission testing (AET) has been used during the cool down of equipment for detection of HTHA (Ref 82, 140). This was possible as thermal gradients typically produce adequate stress to "activate" HTHA damage, i.e., to cause the fissures to form or propagate so that they can be detected by AET. AET does not give much information about the crack (in terms of location and characteristics) and does not detect static or inactive defects (Ref 140). Some researchers have used AET to examine the dynamic fracture mechanism during a fracture toughness test in hydrogen attacked steel at different stages of attack (Ref 141, 142) or to correlate acoustic emission signal behavior with the degree of HTHA in a tension test (Ref 143).

There are also reports of engaging magnetic measurements (Ref 144) and an improved electromagnetic method (eddy current) with special transducer and lens (Ref 145) for detecting HTHA damage with limited success.

Field metallography and replication may be useful only when access to the inside surface is possible and granted, as the damage is typically close to the inside surface of the vessel or tubular. Even access to the inside surface may only show surface decarburization (if present), while micro-fissures are typically further inside the wall (Ref 82).

\subsection{Destructive Examination and Testing}

Destructive methods by cutting pieces out for metallographic examination and/or mechanical testing are the most reliable techniques for evaluation of HTHA (although not always feasible for equipment in service). A typical metallographic examination involves preparing a metallographic crosssection through the region of interest (e.g., a cross-weld sample that includes weld metal, the HAZ's and the base metals on both sides of the weld) and examining it in an optical microscope at a magnification of approximately 50-200 $\times$ first in the as-polished condition (Ref 62, 19, 146). This may be preferred to examining the microstructure in the etched condition, as in the case of a ferrite-pearlite microstructure some intergranular microcracks may not be readily discernible adjacent to the pearlite colonies. It should be added that macroetching of the polished sample by hot acid (i.e., immersion in $50 \% \mathrm{HCl}$ at $160-175^{\circ} \mathrm{F}\left(70-80{ }^{\circ} \mathrm{C}\right)$ for $\left.30 \mathrm{~min}\right)$ to evaluate the depth of fissuring attack was part of the evaluation in the past (Ref 16, 147).

Microstructural examination may be continued in a scanning electron microscope (SEM) to look for grain-boundary bubbles on the metallographic sample at higher magnifications (e.g., $2000-10,000 \times)$. It should be noted that examination of cracks at lower magnifications (e.g., 100-300×) in the SEM using secondary electron imaging shows distribution of fissures (especially those adjacent to pearlite colonies) better than an optical microscope due to its enhanced topographical contrast. Transmission electron microscope (TEM) has also been employed for the examination of the damage by some researchers at least since 1974 (Ref 50, 79, 148, 149). However, TEM is not a very useful or practical tool due to the difficulties in preparing the thin-foil sample, the high level of expertise needed for operating a TEM and the fact that a tiny TEM sample may not be representative of the physical component. TEM extraction replicas, however, are useful for examining the carbides through electron diffraction and energydispersive x-ray (EDX) spectroscopy (Ref 109, 150).

Breaking the damaged regions cryogenically to open some cracks [referred to as "cryo-cracking" by some researchers (Ref 151)] and examining the crack surfaces in SEM is another step in metallurgical evaluation of HTHA damage (Ref 152). The HTHA damaged regions may exhibit areas of dimpled intergranular fracture as a result of formation of numerous (methane) bubbles on the grain boundary surfaces (Ref 151, 153).

Note that as early as in 1950s, it has been shown that reliance on microstructural alteration as evidence of HTHA is not advisable as some samples that showed no visible microstructural alterations (decarburization or fissuring) failed some mechanical tests (e.g., 180-degree bend test or impact test) (Ref 62).

Several parameters or measurements have been used to evaluate the degree or percentage of HTHA damage. This includes percentage of reduction-of-area loss in a tension test (Ref 19), chemical hydrogen analysis in combination with other tests (such as metallographic and UT examinations (Ref 154)) and Charpy V-notch (CVN) impact test (Ref 151). Dilatometry is the most accurate method used in laboratory investigations to trace the stage of HTHA damage, when coupled with metallographic examination (Ref 25). Use of differential dilatometers with a detectability of $10^{-5}$ delta V/V (Ref 45) or capacitance dilatometers with a sensitivity of less than 10-6 
delta $\mathrm{L} / \mathrm{L}$ in monitoring the swelling or expansion rates as a function of test variables, especially at the early stages of attack (i.e., delta $\mathrm{V} / \mathrm{V}=10^{-5}-10^{-2}$ ), were reported in late $1970 \mathrm{~s}$.

Mechanical testing can include (slow-strain) tension test, bend test, hardness test, CVN impact test and crack tip opening displacement. Typically, these tests are complemented by SEM examination to comment on the presence and location of bubbles or cavities. Percentage of the change in mechanical property under the investigation is a quantitative measure of HTHA damage. It has been known for decades that different tests have different sensitivity to the onset of damage. Allen et al. found that bending properties showed a more definitive indication of attack than the tensile properties (Ref 21). At early stages of attack, a CVN test may show HTHA damage before a tension test does and both may show deterioration before microstructural alterations can be observed (Ref 16). Shewmon observed that loss of reduction-in-area during a room-temperature tension test is "the most striking indication of hydrogen attack" (Ref 155). Note that changes in mechanical properties may depend on the combined effects of different types of microstructural damage/alterations. For instance, significant decarburization can result in a decrease in strength/hardness and an increase in elongation due to softening, whereas grainboundary fissuring can result in a decrease of elongation as well as strength. Hardness may increase in affected areas due to the presence or saturation of atomic hydrogen. Instances of local hardness increase has been reported in some literature (e.g., (Ref 71, 156)).

Lundin et al. (Ref 22) suggested that CVN toughness can be correlated to the degree of HTHA damage and this relationship can be used in equipment safety decision making. They measured the amount of hydrogen released after elevatedtemperature decomposition of the trapped methane to quantify the degree of HTHA damage and correlate with the CVN toughness. This led to the development of "Toughness Prediction Factors" for C-0.5Mo steel in HTHA susceptible environment. Experimental results show that the trapped methane concentration and the CVN absorbed energy have virtually a reverse linear relationship (Ref 49).

Shewmon and Xue (Ref 157) used a "wedge-opening loading " specimen to evaluate the effect of HTHA damage on crack growth rate of carbon steel under steady applied load. They studied the relationship between pressure and temperature (separately) with crack growth rate. Later, Shewmon and Xue (Ref 158) used a modified wedge-opening loading specimen to evaluate the "stress-assisted hydrogen attack cracking " of a $\mathrm{Cr}$ Mo steel. They reported a critical/threshold stress intensity at a given temperature, above which crack growth increased rapidly with increasing stress intensity.

HTHA parameters $P_{\mathrm{V}}$ and $P_{\mathrm{W}}$ (see Section 16.3) have been used as a degree of HTHA exposure to sort and tabulate/graph destructive or non-destructive test results (Ref 159). This is similar to the way Larson-Millar parameter can be used for comparing different tempering or other thermal exposure test results.

\subsection{Criteria for Attack}

A problem with most established boundary limits, such as the curves presented by Naumann, Schuyten and Nelson, is that the criterion for attack is not clearly stated. These curves separate conditions conducive to hydrogen attack from noattack (or unsafe from safe or unsatisfactory from satisfactory).
The criteria for attack could be microscopic/macroscopic observations (that could range from the observation of bubbles to fissures and to macrocracks/failure) or property change (e.g., a certain level of drop in carbon concentration or mechanical properties).

The Japan Pressure Vessel Research Committee has used two experimental criteria for HTHA (Ref 160): (1) a drop exceeding $15 \%$ in CVN absorbed energy or tensile reduction in area; (2) microscopic observation of bubbles or grain-boundary fissures at a magnification of $2000 \times$. Criteria for attack should be standardized globally.

\section{Laboratory HTHA Tests and Simulations}

Laboratory tests and simulations have been carried out over the last century to determine the effects of temperature, pressure, time (and the kinetics of damage), applied stress, prior cold work, weld thermal cycle, heat treatment, composition, etc. on the HTHA susceptibility of steel. This section describes some of the devices, specimen designs and test methodologies that the researchers have used for successful assessment of HTHA.

Autoclaves have been used to determine the sensitivity of steel grades to HTHA under a pair of constant temperature and hydrogen pressure (Ref 161). The material would then be examined metallographically to evaluate the state of cavities. This type of test has a potential of becoming standardized, especially if the hydrogen-exposed sample is tested mechanically, which is quantitative and more sensitive to damage initiation than microstructural examination.

Tsubakino et al. (Ref 162) reported an electrochemical method of charging hydrogen into carbon steel for the evaluation of HTHA at a temperature of $380-500{ }^{\circ} \mathrm{C}$. They used a molten $\mathrm{NaOH}$ electrolyte and maintained a cathodic potential of $-1.7 \mathrm{~V}$ (against $\mathrm{Air} / \mathrm{ZrO}_{2}$ ), considered to create a current density equivalent to a hydrogen pressure of 4-8 $\mathrm{MPa}$. They also reported using a molten $\mathrm{NaOH}$ electrolyte for monitoring the permeated hydrogen concentration in a hydrodesulphurizing plant at $400{ }^{\circ} \mathrm{C}(673 \mathrm{~K})$, which they considered highly reliable (Ref 163).

An interesting test specimen used in the past is the "tensile test vessel" used by Thygeson and Molstad (Ref 76) that was devised originally by Van Ness (1955). The specimen is actually a miniature hydrogen pressure vessel, i.e., the response of the material is being tested under an externally applied axial stress and an internal hydrogen exposure. They also placed some loose wire inside the vessel to examine the non-stressed response of the material as well.

Masaoka et al. (Ref 152) used "critical embrittling temperature" in assessing the effects of chemical composition (both alloying elements and impurities) and heat treatment on HTHA resistance of C-0.5Mo and Cr-Mo steels. Critical embrittling temperature was defined as the temperature corresponding to $50 \%$ drop in reduction of area upon tension testing after exposure to hydrogen at an elevated temperature. This temperature may be above or below the Nelson curve for the material based on many factors such as material composition, heattreatment and applied stress magnitude.

In order to correlate the tempering parameter effect with the type and stability of carbides in a low-alloy steel (i.e., $0.5 \mathrm{Mo}$ or $\mathrm{Cr}-\mathrm{Mo}$ ), some researchers extract the carbides electrolytically 
for EDX and x-ray diffraction (XRD) analyses. To extract the carbides, Chiba et al. (Ref 111) dissolved the samples after heat treatment in an electrolyte of methanol with $10 \%$ acetylacetone and $1 \%$ tetramethyl ammoniumchloride and by applying an electrode potential of $-10 \mathrm{mV}$ (vs. SCE). Some other researchers (e.g., George et al. (Ref 150)) have used TEM carbon extraction replica for EDX and electron diffraction analyses of the extracted carbides.

Since atomic hydrogen dissolved in the matrix can cause reversible $\mathrm{HE}$, any test on a specimen exposed to hydrogen at high temperature to evaluate HTHA should be performed after the specimens are degassed by baking hydrogen out to separate the effects of the two damage mechanisms (Ref 21). This can be achieved by annealing the sample in vacuum, for instance at $250{ }^{\circ} \mathrm{C}$ for $3 \mathrm{~h}$ (Ref 56).

Another important point is comparing the results of the tests carried out after exposure to hydrogen to the same tests carried out after exposure to argon, as performed by many researchers (Ref 4, 56, 77, 80, 85, 102, 164, 165). This will separate the effects of HTHA from other types of elevated-temperature metallurgical damage, such as creep (in stressed samples) and aging. For instance, through such a comparison, George et al. (Ref 150) concluded that an embrittlement of a $3 \mathrm{Cr}-1 \mathrm{Mo}-1 \mathrm{Ni}$ (up to $22 \%$ decrease in upper shelf impact energy and 65-100 ${ }^{\circ} \mathrm{C}$ increase in transition temperature) upon exposure to 14 $17 \mathrm{MPa}$ hydrogen at $550-600{ }^{\circ} \mathrm{C}$ for $1000 \mathrm{~h}$ was due to a type of "thermal damage" as opposed to HTHA. One can also compare a certain parameter (such as CVN absorbed energy) upon exposure to $\mathrm{H}_{2}$ at elevated temperature once to that without hydrogen and once to that of the as-received condition, as reported by Shida et al. (Ref 105, 106). Some researchers have carried out the parallel comparative tests in air instead of argon (Ref 106, 159). In principle, testing in argon is preferred to testing in air due to the possible involvement of oxidation and decarburization in air.

Ando et al. (Ref 166) and Nishimura et al. (Ref 167) developed ceramic sensors that measured the permeation of hydrogen into the steel at an elevated temperature and were used to determine the hydrogen content and diffusivity in the steel.

\section{Prevention and Mitigation}

Mitigation of HTHA can be achieved by keeping operating conditions within the limits known to be safe (particularly controlling the temperature using the Nelson curves; see Section 16), proper heat treatment (e.g., tempering after cooling from the austenitization temperature or PWHT after welding), upgrading material to more HTHA resistant alloys, and use of liners or coatings that either are resistant to HTHA and reduce hydrogen permeation or act as insulators and reduce the metal temperature (Ref 72). Archakov (Ref 168) distinguishes between three types of barriers, namely thermal barriers, diffusion barriers (e.g., films of oxides, carbides or nitrides) and permeation barrier (e.g., stainless steel clad).

A crucial point is the use of "actual" operating conditions of a component in risk assessment. Direct temperature measurement and knowledge of temporary conditions and upsets have been emphasized in the Tesoro incident report (Ref 1).

By using HTHA resistant cladding, the concentration of hydrogen diffusing into the susceptible metal is reduced due to the significantly lower hydrogen permeability of the liner. Archakov and Grebeshkova (Ref 169) reported that, among common industrial materials, hydrogen permeability increases from aluminum to copper, nickel, stainless steel and carbon steel. They provide mathematical equations (based on Fick's Laws) to calculate hydrogen pressure at the interface of the two metals. By knowing the pressure and temperature at the clad interface, then HTHA safety can be evaluated using the Nelson curves for the backing.

Note that liners may initially prevent the attack but may cause accumulation of hydrogen molecules that form at the interface of the liner and the vessel, since hydrogen can typically diffuse through the liner (Ref 4). This can lead to bulging and disbondment of the liner or clad material, which compromises the mitigating effect and leads to HTHA at the bulged areas. One solution is to drill tiny "weep holes" in the vessel to prevent hydrogen pressure buildup at the interface (Ref 37, 170). The use of metallurgically bonded austenitic stainless steel liners inside carbon steel vessels is particularly effective, not only due to the high corrosion resistance of austenitic stainless steel, but also because it provides an order of magnitude lower hydrogen flux than the ferritic material due to the lower diffusivity of hydrogen and its higher solubility in austenite (Ref 135). Sakai et al. (Ref 81) estimated that the use of austenitic stainless steel overlay increases the critical temperature for $2.25 \mathrm{Cr}-1 \mathrm{Mo}$ steel by approximately $50^{\circ} \mathrm{F}$ $\left(28^{\circ} \mathrm{C}\right)$.

Coatings can have positive, semi-positive or even negative effects. Non-occlusive coatings (such as zinc, tin, aluminum and cadmium) are semi-permeable to hydrogen, whereas tungsten and gold are completely impermeable to hydrogen (Ref 4). Some coatings/films (e.g., $\mathrm{NaSiO}_{4}$ ) can accelerate hydrogen absorption into steel, while others (e.g., $\mathrm{Na}_{2} \mathrm{CrO}_{4}$ ) can inhibit that (Ref 8).

Another important mitigation measure is the control of operating conditions and regular inspections. As pointed out by Rothwell (Ref 8), for equipment operating within 50 psi or $50{ }^{\circ} \mathrm{F}\left(28{ }^{\circ} \mathrm{C}\right)$ under the API RP 941-2016 Nelson curves, regular direct measurements of temperature and pressure and regular NDE is highly recommended. Most susceptible spots in terms of temperature or applied/residual stress (e.g., welds) should be identified and monitored carefully.

With a stricter control of operating conditions and regular inspections, equipment with HTHA damage may be able to continue operation until a proper time for replacement. As a last resort, refractory insulator was attached to the inside surface of a C-0.5Mo steel vessel with HTHA problems (after disbondment of the original stainless steel clad) to minimize the metal temperature. This mitigation resulted in HTHA arrest reportedly, until the vessel was replaced (Ref 170). Refractory liners are generally not a reliable solution as they can degrade/crack easily and produce hot spots on the vessel (Ref 171) as well as a direct path of contact between the process fluid and the vessel inside surface.

\section{Recovery or Healing}

Although HTHA is considered an irreversible damage mechanism, some recovery of properties upon high temperature annealing has been reported in the literature. Weiner (Ref 19) reported in 1961 that (partial to significant) recovery in terms of 
ductility (but not strength) may be achieved upon heating the HTHA affected components at $1250-1650{ }^{\circ} \mathrm{F}\left(680-900{ }^{\circ} \mathrm{C}\right)$ for a period ranging from $0.5 \mathrm{~h}$ to several hours, as long as the component has not been drastically damaged. Metallographic examination of the recovered material revealed reduced grain boundary damage.

It has been known that methane gas can decompose to carbon and hydrogen at temperatures above $450{ }^{\circ} \mathrm{C}(\operatorname{Ref} 154)$. Based on this fact, Dong et al. (Ref 172) proposed an HTHA crack healing heat treatment by heating the equipment cyclically between the room temperature and $1000{ }^{\circ} \mathrm{C}(5$ cycles with a total time of $10 \mathrm{~h}$ ). By applying this heat treatment to carbon steel samples previously exposed to $12 \mathrm{MPa}_{2}$ at $350-500{ }^{\circ} \mathrm{C}$ for $120 \mathrm{~h}$, they reported that all fissures of 10 microns and under disappeared. They also tried isothermal heating at a range of temperature from 650 to $1000{ }^{\circ} \mathrm{C}$. Healing occurred at the higher temperatures but not adequately at the lower temperatures.

It should be added that heat treatment above the lower critical temperature of steel (i.e., $\mathrm{A}_{1}$, approximately $720{ }^{\circ} \mathrm{C}$ or $1330^{\circ} \mathrm{F}$ ) can drastically change the microstructure due to austenitization and grain growth, which would significantly alter the original mechanical properties. Therefore, recovery and healing of HTHA through very high-temperature annealing may not be very practical.

\section{Prediction}

\subsection{The Nelson Curves}

The main tool available to the industry for assessing susceptibility of a component to HTHA is what is known as the Nelson curves. The curves, as shown in Fig. 4, were first published in 1949 by Nelson (Ref 37) and were later incorporated by American Petroleum Institute in 1970 as API Publication 941 (Ref 173). Nelson commented that the limits are to be used for design. They were initially established based on satisfactory and unsatisfactory industry data. As mentioned by Nelson (Ref 122), over 22 revisions to the charts (or curves) were made in 7 years, upon adjustments made to the initial industry data after more data was gathered over time. These adjustment and shifts continued over years (Ref 97), as one would expect from empirically driven industrial data. Cantwell provides a historical review of the initial development of API 941 in 1970 and the subsequent changes in the next three editions in 1977, 1983 and 1990 (Ref 174).

Nelson curves have been used vastly (and generally successfully) around the world for two main purposes. Firstly for design, i.e., either select the steel type for a given pair of operating conditions ( $T$ and $P$ ) or limiting one of the operating conditions based on the other parameter and the steel type. Secondly for inspection planning based on how close the operating conditions are to the curves and upon establishing some criteria (Ref 33). See Section 16.3 for the second purpose.

It should be pointed out that the idea behind the curves originated decades earlier. As summarized by Fletcher and Elsa (Ref 4), Inglis and Andrews published limits of temperature and pressure for different types of steel subjected to HTHA in 1933. The first set of curves for HTHA of carbon steel and low-alloy chromium steel $(1,2,3$ and $6 \% \mathrm{Cr}$ ) was published by Naumenn (Ref 14,15$)$ in Germany in 1938. These curves were based on laboratory experiments upon $100 \mathrm{~h}$ of exposure as opposed to industry long-term data in the Nelson curves. Naumann had anticipated lower curves for long-term exposures.

It also appears that it was Schuyten from Shell Development Company in USA who first published a "boundary condition" curve for carbon steel upon exposure to hydrogen at longer

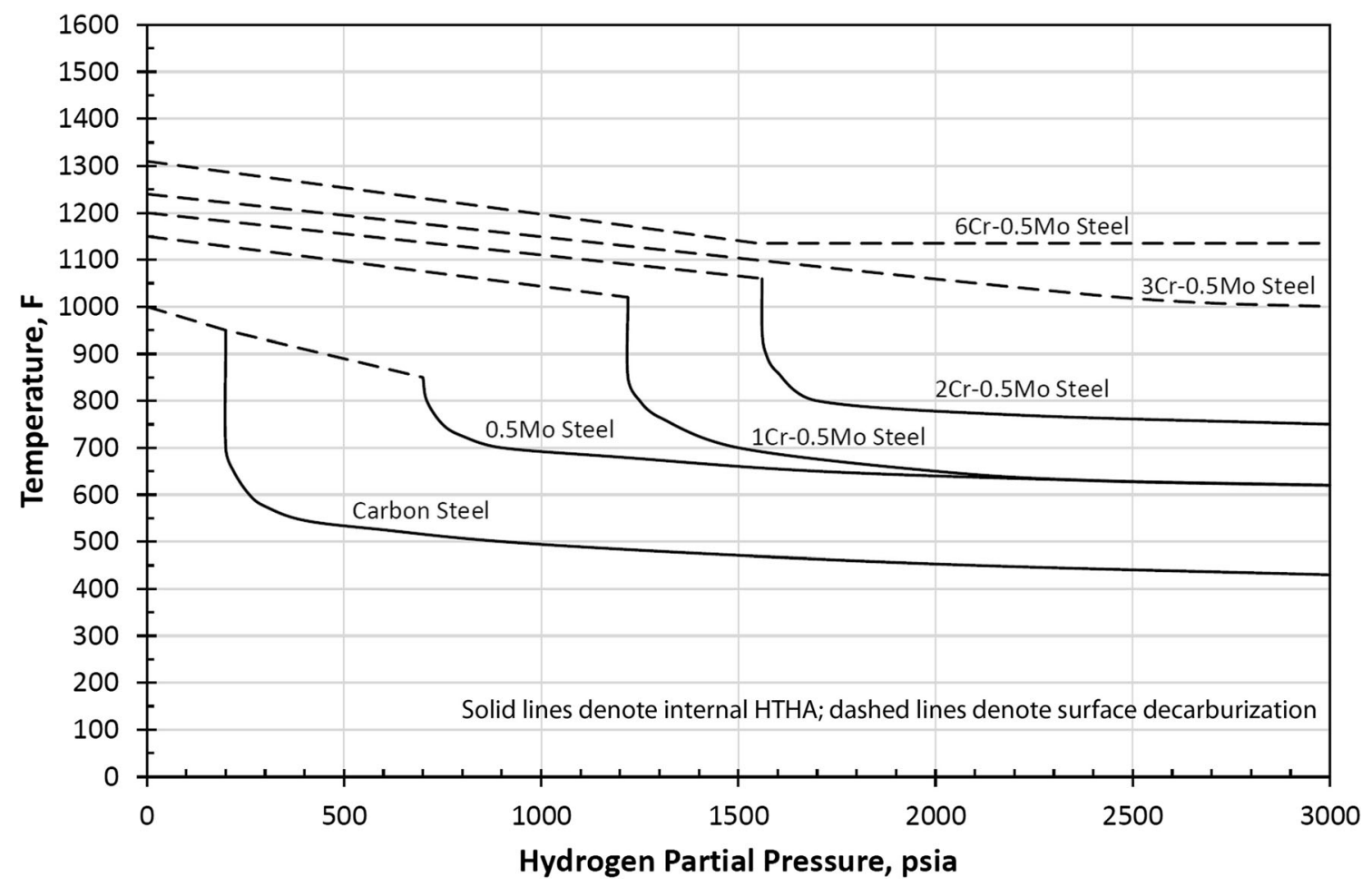

Figure 4 The first published operating limit curves by Nelson (Ref 37) (redrawn from the original graph) 
exposure times than 100 h. In 1946-1947, he presented two curves for carbon steel (Ref 16): Curve 1 (higher) was Naumann's tests for $100 \mathrm{~h}$; Curve 2 (lower) was a compilation of the "long-term" results of tests by several researchers. The curves were lines passed through points representing "attack" or "no attack". Nelson reports that Shell had worked on the development of long-term limits for their specific operations (i.e., at temperatures much lower than those reported by Naumann) before WWII. More data were obtained at Shell during WWII upon an increase in production demands. Nelson also refers to the technical data received from the Germans during the war (Ref 37).

16.1.1 Carbon Steel Curve. Between 2010 and 2014, there were reports of a series of failures of carbon steel weldments without PWHT around the world that operated in the safe zone of the API RP 941 Nelson curve. This included refineries in the USA (e.g., (Ref 1, 68)) and Europe (e.g., (Ref 117)). Following the recommendations from these investigations, a curve for non-PWHT'ed carbon steel material was added to the Nelson curves in 2015 and released in the $8^{\text {th }}$ Edition (2016) of the API publication. This curve is approximately $50 \mathrm{psi}$ to the left of the preceding carbon steel curve at the vertical leg and approximately $50{ }^{\circ} \mathrm{F}$ lower at the horizontal leg. (The distance is slightly larger between the knees of the curves.) It should be noted that Nelson did distinguish between the welded and non-welded carbon steel curves as early as in 1960 (Ref 175). The vertical leg of the curve was set at 200 psia hydrogen partial pressure for "not welded" carbon steel and at 100 psia for "welded or hot bent" (Ref 97, 176). However, this distinction was lost in the second edition of API Publication 941 (Ref 42) when the vertical leg for both conditions was set to the lower limit (i.e., 100 psia). Table 2 summarizes these changes for carbon steel over time.

It should be pointed out that many failures of the equipment (nominally) operating in the safe zone is perhaps due to the fact that the actual operating conditions are higher than the nominal values, even for a relatively short period of time (Ref 68, 71). This has even been considered plausible for the catastrophic Tesoro Petroleum refinery incident in 2010 (Ref 1, 8). In fact, one of the recommendations of the investigation report on the Tesoro incident, as pertains to API RP 941, was a requirement for the use of the actual operating conditions in failure assessments (Ref 1).

16.1.2 0.5Mo Steel Curve. $\quad 0.5 \%$ Molybdenum steel (i.e., $\mathrm{C}-0.5 \mathrm{Mo}$ and $\mathrm{Mn}-0.5 \mathrm{Mo}$ ) is perhaps the only type of steel that was subjected to a roller-coaster experience. In 1965, Nelson raised the $0.5 \mathrm{Mo}$ curve approximately $50{ }^{\circ} \mathrm{F}$ between 725 psia and 1500 psia, due to satisfactory industry results (Ref 78, 97). This left a discontinuity on the curve at 725 psia and $840^{\circ} \mathrm{F}$ (that was originally the break point between the vertical leg of the HTHA curve and decarburization shelf for $0.5 \mathrm{Mo}$ steel), thought to be rectified in the future with more data gathering. He also added several sub-curves between the carbon steel curve and $0.5 \mathrm{Mo}$ steel curve (i.e., for $0.1,0.2,0.3$ and $0.4 \%$ Mo steels) in an additional chart to show the effect of trace alloying elements. In 1966 (Ref 176), Nelson removed the discontinuity in $0.5 \mathrm{Mo}$ curve (at $725 \mathrm{psia}$ and $840{ }^{\circ} \mathrm{F}$ ) and instead added a $0.25 \mathrm{Mo}$ steel curve to the main chart (for the first and the last time).

In the next years, however, several reports of failures under the curve of $0.5 \mathrm{Mo}$ steel were received and as a result the curve was lowered about $60{ }^{\circ} \mathrm{F}\left(\sim 30^{\circ} \mathrm{C}\right)$ in the 2 nd edition of API Publication 941 in (Ref 174). As pointed out by Baumert et al. (Ref 128), despite continued failure reports below the revised curve, the $3^{\text {rd }}$ edition of API 941 (1983) did not lower the curve for $0.5 \mathrm{Mo}$ steel any further and only suggested caution when this type of steel is used in catalytic reformers. Eventually, further unexpected failures significantly under the revised curve led to the removal of the $0.5 \mathrm{Mo}$ steel curve from the main diagram in the 4th edition of API RP 941 (1990) and its separate presentation so that the users apply caution when dealing with 0.5 Mo steel (Ref 47).

The problem with $0.5 \mathrm{Mo}$ steel is suspected to be due to the stability of the carbides, which depends on the exact composition (rather than nominal) and the thermal history. Chiba et al. (Ref 112) showed through XRD analysis that the main carbide type in $0.5 \mathrm{Mo}$ steel was $\mathrm{M}_{3} \mathrm{C}$ (similar to cementite), which has a relatively low stability. In the base metal, there was also the more stable $\mathrm{M}_{23} \mathrm{C}_{6}$ type whose relative amount increased with increasing tempering temperature (with a sufficient time of at least $5 \mathrm{~h}$ ). But in the simulated HAZ, the predominant type remained to be $\mathrm{M}_{3} \mathrm{C}$ even upon tempering between 650 and $700{ }^{\circ} \mathrm{C}$ and the only difference was the increase of molybdenum concentration in the carbides with increasing tempering temperature. They found that a tempering temperature of $650{ }^{\circ} \mathrm{C}$ provides the optimum effects of carbide stability and distribution with regards to $0.5 \mathrm{Mo}$ susceptibly to HTHA.

Kimura et al. (Ref 103, 177, 178), however, reported a different account for $\mathrm{M}_{23} \mathrm{C}_{6}$ carbides in $0.5 \mathrm{Mo}$ steels. In the past, there were two options for the heat treatment of $0.5 \mathrm{Mo}$ steel, i.e., annealing and normalizing-and-tempering. Kimura et al. found that slow cooling from austenitizing temperature associated with annealing can result in precipitation of

Table 2 Vertical leg limit evolution of the nelson curve for carbon steel

\begin{tabular}{|c|c|c|c|}
\hline \multirow[b]{2}{*}{ Year } & \multicolumn{2}{|c|}{ Pressure, psia } & \multirow[b]{2}{*}{ Note } \\
\hline & $\begin{array}{l}\text { Welded (or hot } \\
\text { bent) }\end{array}$ & $\begin{array}{l}\text { Not-welded or welded-and- } \\
\text { PWHT'ed }\end{array}$ & \\
\hline 1949 & 200 & 200 & The first publication by Nelson on boundary conditions (Ref 37 ) \\
\hline 1965 & 100 & 200 & Nelson made a distinction between the two weld conditions (Ref 97, 175) \\
\hline 1970 & 100 & 200 & API Publication 941, 1st ed. (Ref 173) \\
\hline 1970 & 100 & 100 & $\begin{array}{l}\text { API Publication 941, 2nd ed. (Ref 42), reduced the limit for not-welded or } \\
\text { welded-and-PWHT'ed steel }\end{array}$ \\
\hline 2016 & 50 & 100 & $\begin{array}{l}\text { API RP 941, } 8 \text { th ed. (Ref } 2) \text {, reduced the vertical and horizontal limits for } \\
\text { welded steel }\left(50 \text { psia and } 50^{\circ} \mathrm{F}\right)\end{array}$ \\
\hline
\end{tabular}


"abnormal quasi- $\mathrm{M}_{23} \mathrm{C}_{6}$ globular carbides" in $0.5 \mathrm{Mo}$ with a ferrite-pearlite microstructure and significant reduction of HTHA resistance. The quasi- $\mathrm{M}_{23} \mathrm{C}_{6}$ carbide in $0.5 \mathrm{Mo}$ steel was thought to be different from the thermally stable $\mathrm{M}_{23} \mathrm{C}_{6}$ carbides in Cr-Mo steels. Normalized $0.5 \mathrm{Mo}$ steel microstructure comprised $\mathrm{M}_{3} \mathrm{C}$ carbides in a bainitic microstructure and showed higher HTHA resistance. Upon examining samples from 156 heats from the industry, they observed that all failures below the Nelson curve for $0.5 \mathrm{Mo}$ steel were associated with the slow-cooling heat treatment and the massive globular quasi$\mathrm{M}_{23} \mathrm{C}_{6}$ carbides. In fact, they provided a correlation between the percentage of $\mathrm{M}_{23} \mathrm{C}_{6}$ carbides and deviation from the Nelson curve as well as effective curves between the Nelson curves for $0.5 \mathrm{Mo}$ and carbon steel for $20,40,60,80$ and $100 \%$ $\mathrm{M}_{23} \mathrm{C}_{6}$ carbides (Ref 179).

16.1.3 Incubation Curves. Nelson had also provided "time for incipient attack" curves (a.k.a. "incubation curves") for carbon steel and $0.5 \mathrm{Mo}$ steel that would show the effect of time on the $T-P$ curves (Ref 97). In practice, it would serve to predict the effect of unplanned upsets or intentional short-term increase in temperature or pressure on HTHA damage; one could determine how long they have until detectable HTHA damage occurs after an upset or excursion. It should be added that the incubation curves for $0.5 \mathrm{Mo}$ steel above $100 \mathrm{~h}$ were estimates based on the corresponding curves for carbon steel. Some researchers found that the Nelson's incubation curves of 100-1000 $\mathrm{h}$ for carbon steel in API 941-1977 (i.e., the 2nd edition) to be very conservative. For instance, Pishko et al. (Ref 99) found through laboratory tests that the incubation time in their experiments on carbon steel at $375{ }^{\circ} \mathrm{C}$ and $7.6 \mathrm{MPa} \mathrm{H}_{2}$ was approximately 10 times larger than that predicted by the curves.

See Section 20 for a demonstration of the use of the incubation curves in a case study.

\subsection{Modifications to and Substitutions for the Nelson Curves by Other Researchers}

There has been some criticism towards the empirically obtained Nelson curves. Van der Burg et al. (Ref 36), among many others, believed that the Nelson curves are too simple for determining the susceptibility of a given component to HTHA. They are based on material general type, while neglecting many variables such as exact chemical composition, prior heat treatment (except for the distinction between as-welded and PWHT'ed carbon steel curves), the state of stress (applied and residual) and the time in service. In other words, data of several variables are plotted together. Van der Burg et al. also observed that the Nelson curve predictions are too optimistic for some components (with aggressive, unstable carbides and high levels of stress) and too conservative for other components (under low levels of stress and with very stable carbides due to proper heat treatment).

There have been some attempts to replace or improve on the Nelson curves by various researchers. After the removal of 0.5Mo Nelson curve from API 941-1990, Saugerud et al. (Ref 46) used a swelling model for HTHA and a probabilistic approach to establish "Nelson-type" curves for C-0.5Mo steel with different values of "probability of hydrogen attack", i.e., 10, 50 and 90\%. McLaughlin (Ref 180) also reported the establishment of a new/revised C-0.5Mo curve at ExxonMobil that was $50^{\circ} \mathrm{F}$ and 20 psi above the carbon steel curve of API 941-1990. They also added three partial curves, shifted up at steps of $25^{\circ} \mathrm{F}$ from this "effective Nelson curve" for C-0.5Mo steel, indicating several stages/categories of increased probability of HTHA.

Staats and Buchheim (Ref 181) developed a qualitative approach in 2016 for using adjusted Nelson curves in risk management planning. In addition to the two main parameters $(T$ and $P$ ), the approach took into account cladding type and thickness, corrosion scale and confidence in the operating information. They produced an "adjusted Materials HTHA Resistance curve" based on the Nelson curves and the factors they would consider in each specific case. Decisions were made whether each factor/variable would raise or lower the curve. The drawback of such approach is that it is subjective and arbitrary in nature and does not follow any theoretical or experimental criteria. They concluded that a qualitative method to plan inspection is more practical than trying to calculate HTHA remaining life (which would be very complicated if all important factors were to be considered.)

Also in 2016, Panzarella and Cochran (Ref 49) introduced time-dependent curves (referred to as "Prager curves") to replace the Nelson curves for "safe zone" assessment. Several curves were provided for different periods of time (instead of one single Nelson curve) that were obtained using a probabilistic approach and a Monte Carlo analysis. The sophisticated theoretical model behind these curves is briefly described in Section 17.

\subsection{Hydrogen Attack Tendency Charts}

Upon removal of $0.5 \mathrm{Mo}$ Nelson curve from the 4 th edition of API Publication 941 in 1990, inspection planning of equipment made of $0.5 \mathrm{Mo}$ steel became a crucial task. Hattori and Aikawa (Ref 33) described a method of assessment that included the parameter of time in determining the likelihood of HTHA damage. This would be used for inspection interval planning. They used two HTHA parameters, namely $P_{\mathrm{V}}$ and $P_{\mathrm{W}}$, to derive a hydrogen attack tendency parameter and establish a chart for assessment of HTHA probability (i.e., the "HAT chart"). $\quad P_{\mathrm{V}}=\log \left(P_{\mathrm{H} 2}\right)+3.09 \times 10^{-4} \times T \times(\log$ $(t)+14)$, where $P_{\mathrm{H} 2}$ is hydrogen pressure in $\mathrm{kgf} / \mathrm{cm}^{2}, T$ is operating temperature in Kelvin and $t$ is operating time in hours. $P_{\mathrm{V}}$ is " an expression of time dependency curves ... with temper parameter, proposed by Japan Society of Material Science." $P_{\mathrm{W}}=3 \times \log \left(P_{\mathrm{H} 2}\right)+\log (t)-9.92 \times 10^{3} / T$ and is derived from Shewmon's expansion/swelling rate theoretical equation. Hattori and Aikawa (Ref 33) also proposed limits for the calculated HAT for three intervals of inspection (i.e., 2, 4 and 6-8 years) and proposed empirically determined critical values for both parameters $P_{\mathrm{V}}$ and $P_{\mathrm{W}}$, above which the component would be unsafe, for three conditions: normalizedand-tempered base metal, PWHT'ed simulated HAZ and nonPWHT'ed simulated HAZ.

A HAT chart is a combination of two diagrams (Ref 33): the upper diagram is the Nelson curves (i.e., API Publication 941$1983,3^{\text {rd }}$ edition) for carbon, $0.5 \mathrm{Mo}$ and $1 \mathrm{Cr}-0.5 \mathrm{Mo}$ steels and the lower diagram shows the condition of hydrogen exposure in terms of the HAT parameter $\left(\mathrm{HAT}=3 \times \log \left(P_{\mathrm{H} 2}\right)-9.92 \times\right.$ $10^{3} / T$ ) and time in service (Fig. 5a). Based on the combination of the HAT value and the exposure time, the equipment may be in one of four possible zones: safe, unsafe for as-welded HAZ, unsafe for PWHT'ed HAZ and unsafe for normalized-andtempered base metal. Later in 1997, Hattori (Ref 160) proposed a "corrected HAT $=$ HAT $+\triangle \mathrm{HAT}$ " and an "advanced HAT 

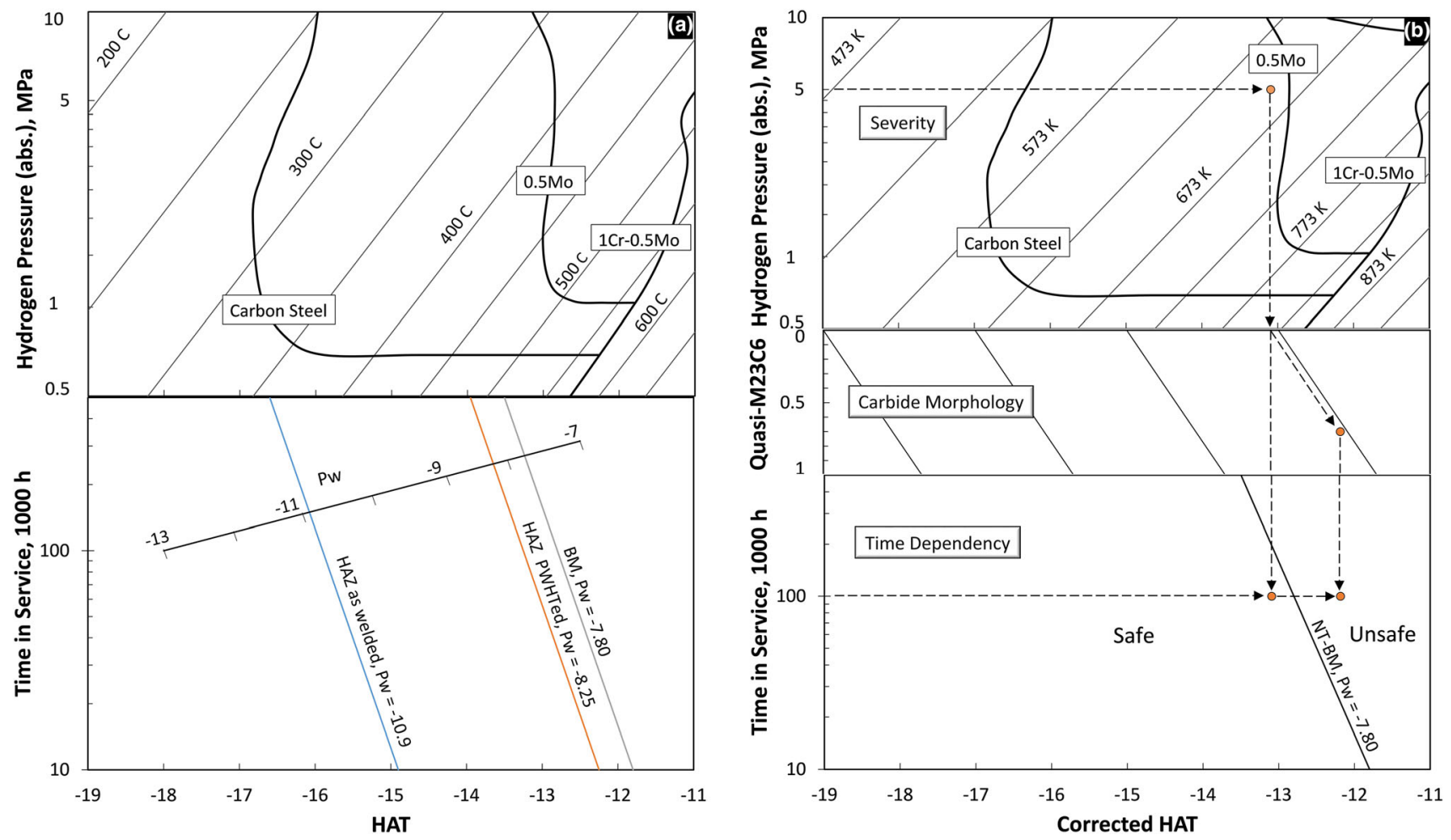

Figure 5 Examples of HAT charts: (a) Original HAT chart (Ref 33); (b) Advanced HAT chart (Ref 160). (Redrawn from the original graphs)

chart" for welded C-0.5Mo steel to take into consideration the effect of unstable carbides. $\Delta \mathrm{HAT}=1.3 \times$ (quasi- $\mathrm{M}_{23} \mathrm{C}_{6}$ ratio) and the quasi- $\mathrm{M}_{23} \mathrm{C}_{6}$ ratio was "quasi- $\mathrm{M}_{23} \mathrm{C}_{6}$ versus $\mathrm{Fe}_{3} \mathrm{C}$ in x-ray diffraction height." (Perhaps, it was meant to be the ratio of quasi- $\mathrm{M}_{23} \mathrm{C}_{6}$ carbide to all carbides or in other words the quasi- $\mathrm{M}_{23} \mathrm{C}_{6}$ carbide fraction.) Plastic replicas were used to extract the carbides non-destructively and determine the ratio/fraction.

The advanced HAT chart comprised a third section between the upper and the lower diagrams to shift the horizontal axis from the original HAT to the corrected HAT and used the critical $P_{\mathrm{W}}$ for normalized-and-tempered base metal as the safe versus unsafe criterion (Fig. 5b). Hattori presents an example, as shown in Fig. 5b, for the use of HAT charts. The case represents a welded $\mathrm{C}-0.5 \mathrm{Mo}$ steel component at a hydrogen partial pressure of $5 \mathrm{MPa}$ and a temperature of $380{ }^{\circ} \mathrm{C}(653 \mathrm{~K})$, which translates to a HAT parameter of -13.1 . Using the original HAT chart (i.e., ignoring the middle section for carbide morphology), the component would be in the safe zone after $100,000 \mathrm{~h}$ of service (based on the base metal critical $P_{\mathrm{W}}$ value, rather than the HAZ critical values in Fig. 5a). However, correcting for the presence of $70 \%$ quasi- $\mathrm{M}_{23} \mathrm{C}_{6}$ carbides, a corrected HAT value of -12.2 is calculated that shifts the point to the unsafe zone even for the base metal. Hattori also proposed a significant procedure in the form of a flow chart "for assuring the structural integrity of $\mathrm{C}-0.5 \mathrm{Mo}$ equipment" in service (Ref 160).

In 1999, Hattori et al. (Ref 182) presented a method to evaluate the HTHA resistance of $0.5 \mathrm{Mo}$ steel equipment nondestructively. This method involved hardness testing at the HAZ (correlatable to $P_{\mathrm{W}}$ threshold value) and identifying carbide morphology of the base metal using plastic extraction replicas and XRD. HAT charts were used to predict the remaining life of the equipment.
Nomura and Sakai (Ref 102) also used the two engineering parameters, $P_{\mathrm{V}}$ and $P_{\mathrm{W}}$, to establish $T-P$ limit curves. They showed the relationship between the parameters and the theoretical equations (directly for $P_{\mathrm{W}}$ and indirectly for $P_{\mathrm{V}}$ ). They also used these parameters for $2.25 \mathrm{Cr}-1 \mathrm{Mo}$ steel, of course with different constant values in the equations. In the end, they showed that the $T-P$ limits (similar to the Nelson's curves) could be established through either a theoretical approach (employing modeling and bubble size measurements) or a parametric method, although they had to choose several parameters in a relatively subjective manner or through a "fitting approach". A detailed report on derivation and application of $P_{\mathrm{W}}$ (for establishing time-dependent boundary curves and to show the effects of applied stress and austenitic overlay parametrically) was published by Sakai et al. (Ref 81 ) in 2000 .

Use of the parameter $P_{\mathrm{V}}$ for establishing inspection and prioritizing guidelines was included in API Publication 581 (2000) Appendix I and implemented by some North American end users in the following years for HTHA assessment of C$0.5 \mathrm{Mo}$ steel equipment (Ref 137,180$)$. However, the parameter and its use in determining the susceptibility to HTHA was removed in the third edition of API RP 581-2016 Section 19, the reason for which was not stated.

\subsection{Depth of Attack}

Bisaro and Geiger (Ref 55) proposed that the maximum depth of attack is a function of hydrogen pressure squared (i.e., $P_{\mathrm{H} 2}{ }^{2}$ ). They could show that hydrogen permeation rate increases over time and reaches a constant value that is a function of temperature. The change in the permeation from the initial value to the constant value (being a function of hydrogen pressure squared) was found to be proportional to the maximum 
depth of attack. However, this discovery did not apparently lead to propose a practical method for determining the depth of attack in service. See Section 12.1 for some non-destructive techniques for determining the depth of attack in service.

\section{Modelling}

The aim of this section is to briefly review, in a chronological order, the attempts made over the last six decades to provide models for HTHA damage without delving into the actual equations or the mathematics, which would be outside the scope of this work. The aim of modeling is to provide a predictive tool that is more accurate than the Nelson curves and takes into considerations parameters in addition to temperature and pressure, e.g., time and stress. Ultimately, models are expected to be used in fitness for service, remaining life assessment and inspection planning. In general, HTHA models can be grouped in two categories (Ref 183): nucleation models and growth models. Growth models are more sophisticated and capable of predictions within an order of magnitude reportedly. They consider methane production rate (represented by a function of cavity size/volume) and either unconstrained or constrained cavity growth by creep.

The first model on incubation thermodynamics and kinetics of the attack was proposed by Weiner (Ref 40, 19). By combining two equations he derived from experimental work (one relating incubation time to temperature and the other to pressure), Weiner formulated an equation for incubation time as a function of pressure and temperature: $t_{0}=C P^{-n} \exp (Q / \mathrm{RT})$. In this equation, $t$ is time in $h, P$ is hydrogen partial pressure in psi, $T$ is temperature in $K$ and $R$ is gas constant $(1.985 \mathrm{Cal} /$ $\mathrm{K}$ mol). He found an activation energy $(Q)$ of $14.6 \mathrm{kCal} / \mathrm{mole}$ for the reaction between carbon and hydrogen in iron as well as the constant values of $C=1.39 \times 10^{6}$ and $n=3$. This equation has been accepted and cited by many researchers and reviewers since then (e.g., (Ref 8, 65, 127)).

Vitovec presented a mathematical analysis of void growth into fissures in Ref 63, considering several steps for hydrogen and carbon reactions and several stages for the growth of cavities. Cavity growth mechanisms include surface tension, vacancy diffusion and dislocation movements. The last two items are creep deformation mechanisms; dislocations become the active mechanism after a certain degree of cavity growth, i.e., when they are "large". The model explained for incubation time and the effect of stress on HTHA; the effects of internal hydrogen pressure inside the cavities and the external stress were "additively superimposed".

For the next 2 decades, Vitovec continued working on HTHA modeling (Ref 183) and in 1982 (Ref 65) he considered eight reaction steps from adsorption of hydrogen atom at the surface and dissolution of carbides to the final reaction between $\mathrm{H}$ and $\mathrm{C}$ inside the voids. A goal in modeling is to find the most time-controlling step to establish a kinetic analysis, which in Vitovec's opinion might depend upon conditions (e.g., humidity facilitates decarburization). It should be added here that Weiner concluded that carbide dissolution is the rate controlling reaction for both incubation and embrittlement. Other researchers identified other sub-mechanisms as the rate controlling factor: Thygeson and Molstad (Ref 76) concluded that the chemical reaction to form methane was the rate-controlling factor. Shewmon (Ref 154) believed that at high temperatures the rate controlling factor in incubation time for fissuring (including bubble formation) is iron diffusion rate migrating away from the growing cavities ("bubbles"), whereas at lower temperatures (and higher pressures) the limiting factor is carbon supply (Ref 155).

Sagues et al. (Ref 25) used kinetic equations for methane generation and cavity growth by creep or grain-boundary diffusion in 1978. The growth mechanism was found to be a function of temperature and pressure. They found that two processes determine the rate of damage: expansion by creep and surface-reaction rates for methane production.

McKimpson and Shewmon (Ref 184) used an in-situ dilatometer in 1981 to investigate the initial-stage kinetics of HTHA in a carbon steel with $0.3 \% \mathrm{C}$. They presented two models for expansion of bubbles resulting in the swelling of steel: a diffusional model and a power-law creep equation. At the earlier stage when the bubbles are small, growth rate based on grain-boundary diffusion model is virtually independent of bubble size. The creep model, on the other hand, predicts a strong dependence on bubble size (at all sizes) as well as methane pressure. They concluded that the constant rate of expansion at the initial stage (as revealed by highly sensitive dilatometry) suggests that the primary mechanism of early expansion under both regimes of the Nelson curves (i.e., lowtemperature, high-pressure regime and high-temperature, lowpressure regime) is grain-boundary diffusion that moves iron atoms away from the bubble surface and is driven by "nearequilibrium internal methane pressure". During the rapid attack, the mechanism of expansion is probably power-law creep.

Similarly, Sundararajan and Shewmon (Ref 104) elaborated on the kinetics of bubble growth through the two parallel processes of power-law dislocation creep and grain-boundary diffusion in 1981. They addressed mechanisms of cavity surface diffusion and matrix accommodation (i.e., creep deformation of the adjacent grains). In grain-boundary diffusion model, bubble growth rate is a linear function of methane pressure. In the creep model, there is a power-law dependence (i.e., exponent $n$ ) on methane pressure as well as bubble size.

Shih and Johnson (Ref 27) calculated the Nelson curve for carbon steel in 1982, based on a void growth model that involved methane reaction kinetics. Methane pressure was considered the driving force and the growth process was considered to be achieved by grain-boundary diffusion at the early stage of damage and later by creep.

Realizing that methane pressure in bubbles is significantly lower than methane fugacity, Odette and Vagarali (Ref 59) presented an "equation-of-state" in 1982 for a better assessment of methane pressure (in carbon steel), which is necessary for a successful modeling.

Parthasarathy (Ref 75) proposed a model in 1985 based on constraint-free growth of methane bubbles along the grain boundaries. The density of the bubbles would determine the HTHA strain rate, which depends on steel composition.

The synergy between creep deformation due to external stress and grain boundary cavitation due to methane bubble growth was numerically investigated in 1987 by Shewmon (Ref 185). The model took into consideration the interaction between bubble pressure, power-law creep and grain-boundary diffusion. It was found that the controlling mechanism is power-law creep under this synergistic condition.

In all these models of late 1970 s and early 1980 s, an equation of the form $\varepsilon=C P_{\mathrm{CH} 4}{ }^{n} \exp (-Q / \mathrm{RT})$ is assumed for 
the incubation strain rate. The value of the exponent $n$, when determined experimentally by dilatometry, indicates the growth mechanism: grain-boundary diffusion $(n=1-2)$, bubble surface diffusion $(n=2-7)$ or dislocation creep $(n=7-10)$ (Ref 6). Note that a similar equation can be considered based on the hydrogen pressure instead of methane pressure, in which case the values of $C, n$ and $Q$ would be different.

Through several papers between 1996 and 1998, Van der Burg, Van der Giessen and colleagues (Ref 34-36, 58, 84, 186) provided models to calculate HTHA damage and the remaining life of the component in HTHA-susceptible service. In 1996, they started from Odette-Vagarali model for methane pressure calculation (Ref 59) and added a model for the growth and coalescence of grain-boundary cavities under macro-scale stress. The model was used for the calculation of the Nelson curves. The results were presented in a non-dimensional form. They expanded on the refinement of their original theoretical model to predict HTHA in their subsequent articles, for instance by presenting a 3-D version of the continuum damage relationship (Ref 34). Time of failure was considered to be reaching a growth ratio of $a / b=0.7$ for penny-shaped cavities of diameter $2 a$ and center-to-center spacing of $2 b$. In 1997, they developed a two-dimensional "continuous damage relation" that was verified by finite-element analysis. The continuous damage relation was based on creep deformation along with two extreme/contrasting modes for HTHA cavitation, i.e., the uniform 'dilatant' cavitation mode (where creep deformation of the grains is negligible) and the non-uniform cavitation mode (with significant grain creep deformation). In 1998, they applied the model to a specific steel (2.25Cr-1Mo) and concluded that cavitation is initially accelerated by stress, whereas it is decelerated by stress later towards the end of the lifetime. The deceleration was thought to be due to a compressive stress development (from the adjacent grains) after re-distribution of internal stresses.

Brear and Church (Ref 187) also attempted in 1996 to include the effect of stress and time in a mechanistic model divided into two types of damage, namely surface decarburization and internal attack. They consider a "degeneration acceleration factor" for the effect of stress after Chuang et al. (Ref 188) as well as a "continuum damage mechanics" formulation to include the effects of creep damage.

In 2000, Schlogl et al. (Ref 189) presented a sub-lattice model to calculate the equilibrium methane pressure as a function of the Gibbs energy of different carbide types $\left(\mathrm{M}_{7} \mathrm{C}_{3}\right.$, $\mathrm{M}_{23} \mathrm{C}_{6}, \mathrm{M}_{6} \mathrm{C}$ and $\mathrm{M}_{2} \mathrm{C}$ ) and volume fractions in $2.25 \mathrm{Cr}-1 \mathrm{Mo}$ steel at different temperatures and estimate the HTHA lifetime. In their 2001 model, Schlogl et al. (Ref 161) coupled methane pressure with cavity growth (unlike some earlier models, in which they were decoupled). The modeling of carbide dissolution and diffusion of metal and carbon allowed considering a non-equilibrium condition, which was thought to be more relevant to industrial situations.

Also in 2001, Van der Geissen and Schlogl (Ref 190) presented a model that described the secondary and tertiary stages of creep coupled with HTHA. As a simplifying assumption, the methane gas was assumed to be in equilibrium condition (unlike in their other paper of 2001 mentioned above). On the other hand, Schlogl and Van der Geissen (Ref 191) presented in 2002 a numerical microstructure model that included a fewer simplifying assumptions (compared to their earlier models). The model was based on variation approach that coupled two processes, namely carbon diffusion in ferrite and the chemical reaction between hydrogen and carbon to form methane. Since the problem became very sophisticated and nonlinear, FEA was used to solve the problem. They acknowledged that the diffusion rate of the metal atom at the dissolving carbide may be the rate controlling process, which they intended to incorporate in future research.

The number of papers on modeling decreased significantly post 2002. As an example of post-2002 paper on modeling for HTHA, the article published in 2011 by Tang et al. (Ref 192) can be mentioned. Through a micromechanics model and computational study, they examined the effect of internal hydrogen and methane pressure on the creep crack growth rate. They took into consideration the effect of traction pressure on the crack flank, which they considered an improvement to their earlier works between 2002 and 2008.

Panzarella and Cochran (Ref 49) developed a mechanistic model of HTHA damage propagation between 2013 and 2016 to be used for FFS evaluation and risk-based inspection decision making. The model was to be proposed as a new Part 15 of API 579-1/ASME FFS-1 in its "upcoming edition". Two sub-models were developed based on "cavity-like" and "crack-like" damage (i.e., volumetric vs. planar). The volumetric damage model is based on the Materials Properties Council Omega creep damage model (API 579, Part 10), modified to account for the internal cavity stress due to methane pressure. Cavities coalesce and form microcracks and macrocracks. The crack-growth model accounts for accelerated crack growth due to hydrogen interaction and is based on a functional analysis and design type of approach that uses empirically measured crack growth rates modified for hydrogen. Two types of damage propagation are considered for the two types of damage. Failure time is defined as the time the equivalent effective (von Mises) stress reaches ultimate tensile strength at any point in the wall. The remaining life is calculated by subtracting the current service life from the calculated failure time. Panzarella and Cochran included many parameters/variables in their models, such as the effect of the "thinning wall" (i.e., reduction of the undamaged wall thickness upon damage accumulation) on hydrogen diffusivity and solubility, materialspecific carbon activity, cladding effect, empirical methane fugacity-pressure relationship as a non-ideal gas, kinetics and dynamics of methane formation, the methane pressure drop upon cavity growth, residual stress and welding effects, and the effects of variable operating cycles. They proposed three levels of inspection for FFS: Level 1 is a screening procedure based on the available preliminary "screening curves", whereas Levels 2 and 3 are two levels of in-depth analysis (applicable to the cases that fail Level 1 screening), in which the shortest perdition of each model (i.e., volumetric vs. planar) is reported as the remaining life.

In 2019, Johnson et al. (Ref 193) briefly presented two methodologies for life assessment of equipment in hydrogen services and a better understanding of the effects of time and stress on the component's life. Through the "screening" method (Model 1), a damage index (DI) was calculated by integrating the damage rate (expressed by Parthasarathy equation and using Orr-Sherby-Dorn interpretation) over a period of time. They provided a guideline for the interpretation of different values of DI and categorization of the condition into various levels of damage likelihood. The "advanced" method predicts damage progress through the wall and the two models separate volumetric damage (Model 2) from crack growth (Model 3). 
In 2020, Chavoshi et al. (Ref 194, 195) presented a continuum damage mechanics model that combined creep and HTHA damage to evaluate FFS of a C-0.5Mo steel after $80,000 \mathrm{~h}$ at $350-500{ }^{\circ} \mathrm{C}$ and under a hydrogen pressure of $4 \mathrm{MPa}$. The model, incorporated into finite element analysis, used hydrogen fugacity and a coupling between Fick's diffusion law and a multi-axial creep ductility model.

It should be added that many modelling approaches yield sophisticated equations that need to be solved through numerical analysis. There have also been some attempts to present simplified closed-form analytical equations, for instance for the incubation period (Ref 196) or the radius and density of bubbles formed during the incubation period of HTHA in quenched-and-tempered 2.25Cr-1 Mo steel (Ref 197), by ignoring some factors. Dadfarnia et al. (Ref 198) also presented in 2019 "a simple (one-dimensional) constraint-based model" by improving the mechanistic models of 1980s. They established Nelson-type curves for the material at different levels of stress; the curves were no longer merely phenomenological (i.e., safe vs. unsafe), but showed time to failure. In comparison with experimental results, the model was reportedly satisfactory under external stress, but not under no external stress.

\section{Fitness for Service and Life Assessment}

The advancements in NDE and modelling have paved the way for development in FFS, remaining life assessment and inspection planning. A major work in this field took place in Japan in the 1970s and 1980s, for instance upon the development of HAT charts (Section 16.3), as reported by Hattori et al. (Ref 182). FFS and life assessment likely gained increasing attention in North America in the 1990s and afterwards (Ref 113, 140). Material Properties Council reported the development of databases on materials exposed to hydrogen (esp. 2.25Cr-1Mo steel) at least since 1990s (but likely starting from the 1970s) to be used for life assessment (Ref 113).

Another major work in this field initiated in 2013 by the Equity Engineering Group through a joint industry project, after development of volumetric and crack-like damage models (Ref 49), as discussed in Section 17. More recently, Wilkowski et al. (Ref 199) discussed the application of leak-before-break concept (that had been developed in the nuclear industry for some decades) to HTHA, particularly for non-PWHT'ed seamless carbon steel piping with girth welds. They provided details on the effect of start-stop positions of different weld passes on crack initiation locations and discussed the effects of inside-initiated crack/flaw on impact toughness (transition temperature) and fracture toughness (crack tip opening displacement and $J-R$ curve) of carbon steel.

Franceschini et al. (Ref 200) used a temperature-based "assessment point margin" (compared to API 941-2016 for PWHT'ed carbon steel) for carbon and $0.5 \mathrm{Mo}$ steel at a given hydrogen partial pressure. The probability of failure was considered high, medium, low or very low when the point was within $0-10,10-30,30-60$ and over $60{ }^{\circ} \mathrm{C}$ of the curve, respectively. Then a consequence of failure categorization was established based on the magnitude of the plant area affected upon a failure. The combination of probability of failure and consequence of failure established the risk matrix. Detailed HTHA evaluation (based on models that took into account time in service and stress in addition to $T$ and $P$ ) was performed on the component with a risk index of high or medium-high.

Prueter et al. (Ref 201) reported a case study of FFS of a vintage C-0.5Mo steel pipe in hydrogen service for over 45 years, in which NDE results and fracture mechanics were used to provide guidelines for safe shutdowns and start-ups to avoid brittle fracture. They determined minimum pressurizing temperature and developed IOW's as short-term measures until the pipe could be replaced.

Johnson et al. (Ref 193) developed two methodologies for life assessment and FFS: screening ("classifying and ranking equipment") and advanced assessment ("predicting throughwall damage progression"). In both methods, the effect of time was considered. In the screening method, a damage index was calculated by integrating the damage rate over a period of time. They presented incubation time (after Weiner's definition and data) versus methane partial pressure in a Larson-Miller form. Then they provided a guideline for different values of DI from incubation to the onset of failure in four categories, D (DI $<0.1), \quad \mathrm{C}(0.1<\mathrm{DI}<0.25)$, B $(0.25<\mathrm{DI}<0.4)$ and A (DI > 0.4). These models neglected the effect of applied stress. In the advanced assessment method, they used the "accepted FFS methods and principles" (as opposed to a true FFS method as per general methodology of API 579-1) to determine "minimum detection limit" of the inspection method and the "critical flaw size". They also separated "volumetric HTHA damage" model from "crack-growth" model.

\section{Related/Accompanying Mechanisms}

In many industrial cases, HTHA is preceded, accompanied, or proceeded by other damage mechanisms, such as corrosion, creep and corrosion fatigue. Since HTHA is a small-scale microscopic damage and the other mechanisms are larger-scale macroscopic damage, HTHA can be overlooked. Corrosion and scale formation/build-up inside the HTHA cracks open to the inside surface can result in "wedge opening" stresses at the tip of the cracks upon cooling (as experienced over shutdowns), which in turn can promote further HTHA crack growth or crack propagation without HTHA (Ref 68). In practice, a complete separation of HTHA from creep, creep-embrittlement, temper embrittlement, stress relaxation cracking and stress relief cracking may be difficult and all such closely related damage mechanisms need to be considered in high-temperature studies for Cr-Mo steels, as demonstrated by Imanaka et al. (Ref 89). The possible interaction among HTHA and two main damage groups, namely creep and metallurgical aging (including grainboundary segregation and precipitation), is symbolically shown in Fig. 6. The main damage mechanisms related to HTHA are briefly reviewed below and the similarities and differences between these mechanisms and HTHA are summarized in Table 3.

\subsection{Decarburization}

Decarburization is an elevated-temperature damage mechanism of carbon steel and low-alloy steel that involves carbide dissolution and atomic carbon diffusion to the surface, where insoluble gasses (e.g., $\mathrm{CH}_{4}$ or $\mathrm{CO} / \mathrm{CO}_{2}$ ) can form. Decarburization results in loss of strength and hardness (Ref 202). As described in Section 10, the main HTHA damage mechanism 
involves internal decarburization and grain-boundary methane bubble formation that leads to fissuring. With the increase of temperature above approximately $600{ }^{\circ} \mathrm{C}$, surface decarburization may replace internal HTHA.

\subsection{Hydrogen Embrittlement}

As described in Section 4, HE is a low-temperature reversible/temporary embrittlement due to absorption of hydrogen that typically affects relatively low-strain-rate properties (such as tensile properties) rather than high-strain-rate properties (such as impact toughness). Proper heat treatment (i.e., annealing at approximately $200-300{ }^{\circ} \mathrm{C}$ for several hours) can result in diffusion of hydrogen out of the material and recovery of the affected properties. In many cases, hydrogen damage (upon cooling (Ref 68)) and HTHA (at the elevated temperature of service) may form on the same equipment. To avoid hydrogen damage upon cooling, adequate time should be given between approximately 300 and $200{ }^{\circ} \mathrm{C}$ for dissolved hydrogen to diffuse out of the metal (Ref 203).

\subsection{Creep}

Creep is the time-dependent deformation of the material under sustained stress at an elevated temperature, typically over $40-50 \%$ of $T_{\mathrm{m}}$, where $T_{\mathrm{m}}$ is the melting temperature of the material in Kelvin (Ref 204). Creep can cause formation of intergranular voids and fissures that would evolve into microcracks and macrocracks that are perpendicular to the applied principal stress. Research shows that there can be significant similarity, interaction and synergy between creep and HTHA. The synergy increases in the low-pressure, high-temperature regime of HTHA (Ref 46). The threshold temperature for creep damage of carbon steel, $0.5 \mathrm{Mo}$ steel, normalized-and-tempered $1.25 \mathrm{Cr}-0.5 \mathrm{Mo}$ steel and $3 \mathrm{Cr}-1 \mathrm{Mo}-\mathrm{V}$ steel are reported to be $345^{\circ} \mathrm{C}\left(650{ }^{\circ} \mathrm{F}\right), 400{ }^{\circ} \mathrm{C}\left(750^{\circ} \mathrm{F}\right), 425^{\circ} \mathrm{C}\left(800^{\circ} \mathrm{F}\right)$ and $440^{\circ} \mathrm{C}\left(825^{\circ} \mathrm{F}\right)$, respectively, in API RP 571 (Ref 204).

The similarity in nucleation of grain-boundary cavities in creep and HTHA is to the extent that some researchers have proposed the same mechanism(s) for both (e.g., the "void sheet" theory by Hirth (Ref 205)). Methane bubbles (i.e., cavities) are believed to grow by creep deformation (Ref 25). As reported by Fletcher and Elsea (Ref 4), Kolgatin et al. (1959) studied the effect of HTHA on creep, when HTHA was significant. HTHA not only decreased endurance (long-time

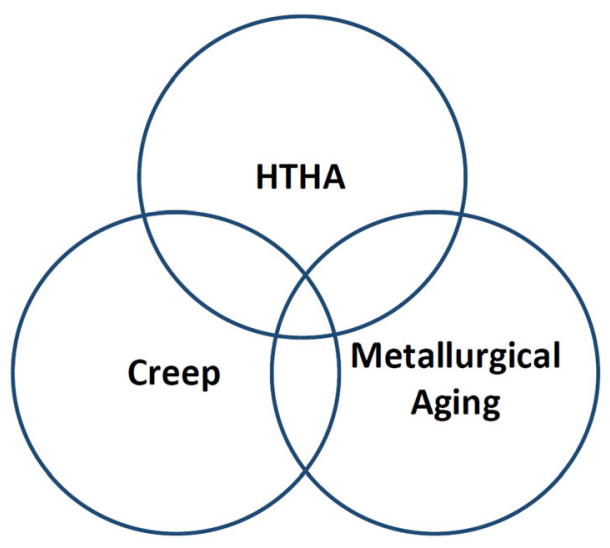

Figure 6 Interactions among three main groups of elevatedtemperature damage mechanisms 
strength) greatly, but also made the failure appear brittle and intergranular without significant amount of deformation (that was present during tests in nitrogen). The effect of stress on HTHA was studied by stress-rupture testing in hydrogen as compared to the test in argon. Hydrogen affected negatively the stress-rupture properties and the rate of hydrogen attack was increased by creep.

Allen et al. (Ref 164) found a loss in the creep rupture strength of normalized mild steel (AISI 1020) in hydrogen at 400-1400 psi and $800-1000{ }^{\circ} \mathrm{F}$, compared to the same test in argon. The relative loss of strength (noted as high as 60\%) typically increased with increasing exposure time (i.e., "time to fracture"). It was also found that the HTHA rate increases by stress during creep and fissures formed mostly along grain boundaries and in the direction normal to the stress, when compared to an unstressed sample in which fissures occurred mostly along MnS inclusions elongated in the rolling direction.

Looney et al. (Ref 77) also investigated the combined effects of HTHA and creep deformation on $2.25 \mathrm{Cr}$-1Mo steel at 600 ${ }^{\circ} \mathrm{C}$ in 1998. They observed that, when tested in hydrogen atmosphere (as compared to that in argon), the creep-rupture deformation decreased drastically, brittle cracks formed at the stress riser notch machined in the tubular and more cavitation formed in the steel. Note that the distinction between HTHA cavities and creep cavities are often difficult (Ref 154).

\subsection{Creep Embrittlement}

In its broadest scope, creep embrittlement can be defined as a reduction in creep or creep-rupture ductility (e.g., as manifested by reduction of elongation or reduction-in-area upon lab testing) due to the involvement of other mechanisms such as strain ageing, grain-boundary segregation/precipitation and HTHA. Therefore, HTHA may be considered as a cause of creep embrittlement, when HTHA and creep are both active. Bruskato (Ref 206) examined the effect of composition on temper embrittlement and creep embrittlement of $2.25 \mathrm{Cr}-1 \mathrm{Mo}$ steel welds. He suspected that impurities and some solutes (especially silicon, manganese, phosphorous and tin, followed by arsenic and antimony) could be an underlying cause of both damage mechanisms.

\subsection{Reheat Cracking}

Reheat cracking is intergranular cracking of an alloy at the elevated temperature of service or during heat treatment without significant deformation (i.e., unlike typical creep). The main cause of reheat cracking is metallurgical alteration of the microstructure, such as grain-boundary segregation and precipitation, or intragranular precipitation and hardening. Stress relaxation cracking is another term that applies to a special type of reheat cracking (when the stress is residual and/ or the stress level decreases upon cracking). If the cracking occurs upon PWHT, the term stress relief cracking is typically used. Vanadium addition to $\mathrm{Cr}-\mathrm{Mo}$ steel to improve elevatedtemperature service cracking (such as creep and HTHA) is believed to compromise the stress relief cracking of the material upon PWHT. To counteract this possible detrimental effect, the sulfur level is typically limited to $0.002 \%(\operatorname{Ref} 90,91)$.

\subsection{Fatigue and Corrosion-Fatigue}

Typically, the internal damage introduced by HTHA facilitates the initiation of a progressive cracking mechanism such as (thermal/mechanical) fatigue or corrosion-fatigue, rather than an instantaneous overload fracture. This becomes a significant factor after Stage 1 HTHA, i.e., upon the formation of fissures. Prior HTHA damage may not affect the fatigue propagation rate significantly (although it does slightly), as studied by Pendse and Ritchie (Ref 207) on quenched-and-tempered Mn-Mo-Ni steel and normalized-and-tempered 2.25Cr-1Mo steel. A case of sulphidation-driven damage after significant HTHA fissuring was examined by McLaughlin et al. (Ref 68).

Suh et al. (Ref 52) studied the effect of HTHA on highfrequency high-cycle fatigue life of Inconel 718 alloy. The fatigue life was decreased $10-20 \%$ after exposure to hydrogen at 100 bar and $300{ }^{\circ} \mathrm{C}$ for $72-100 \mathrm{~h}$. A similar study was carried out by Nahm et al. (Ref 208) on Type 316L stainless steel that showed a similar result.

\section{Case Study}

The failure of the inlet flange of a hydrogenerator reactor piping that was reported elsewhere (Ref 71) in detail is very briefly reviewed here in order to demonstrate the use of the incubation curves (that was not achieved in the previous publication). The use of optical and scanning electron microscopy in both the as-polished and as-etched conditions of a metallographic sample is also demonstrated.

The NPS 6, Schedule 10, non-PWHT'ed carbon steel piping leaked after 47 years of service upon the formation of a circumferential crack on one side of the flange neck (i.e., the extrados). Natural gas with less than 15 ppm $\mathrm{H}_{2} \mathrm{~S}$ (reportedly) and some recycle hydrogen gas ran through the piping at approximately $750{ }^{\circ} \mathrm{F}\left(400{ }^{\circ} \mathrm{C}\right)$ and $400 \mathrm{psi}(2.8 \mathrm{MPa})$. Hydrogen partial pressure was temporarily increased 3 years prior to the failure from 64 psia in average to 116 psia for a period of 275 days.

Metallographic examination revealed a through-wall, scalefilled crack in the sub-critical HAZ of the flange (Fig. 7a). Microscopic examination revealed regions of intergranular fissures with limited internal decarburization (Fig. 7b-e). SEM examination revealed small cavities (i.e., likely methane bubbles) along grain boundaries (Fig. 7f) without noticeable grain distortion (i.e., consistent with HTHA as opposed to creep deformation and cracking). HTHA damage had drastically compromised the Charpy impact toughness of the flange. XRD analysis revealed that the internal scale was $97 \%$ iron sulfide (pyrrhotite and troilite). EDX analysis showed that the scale on the main crack was also made predominantly of iron and sulfur.

It was concluded that HTHA damage formed during the upset period, whereas the crack propagation to failure occurred after return to the normal condition by another damage mechanism (likely a combination of sulfide scale formation/ accumulation inside the cracks and hydrogen embrittlement upon shutdowns). After return to the normal condition, NDE inspection should have been performed on the component periodically and the piping should have been replaced.

Figure 8 shows the pre-2016 API RP 941 incubation curves for carbon steel. No revised incubation curves are yet available for welded (but not PWHT'ed) carbon steel. The solid line in Fig. 8 shows the main pre-2016 carbon steel curve, i.e., the long-term exposure limit [typically considered 200,000 h (Ref 180)], whereas the dashed lines show the limits for some shorter exposure periods. The normal operating condition was 

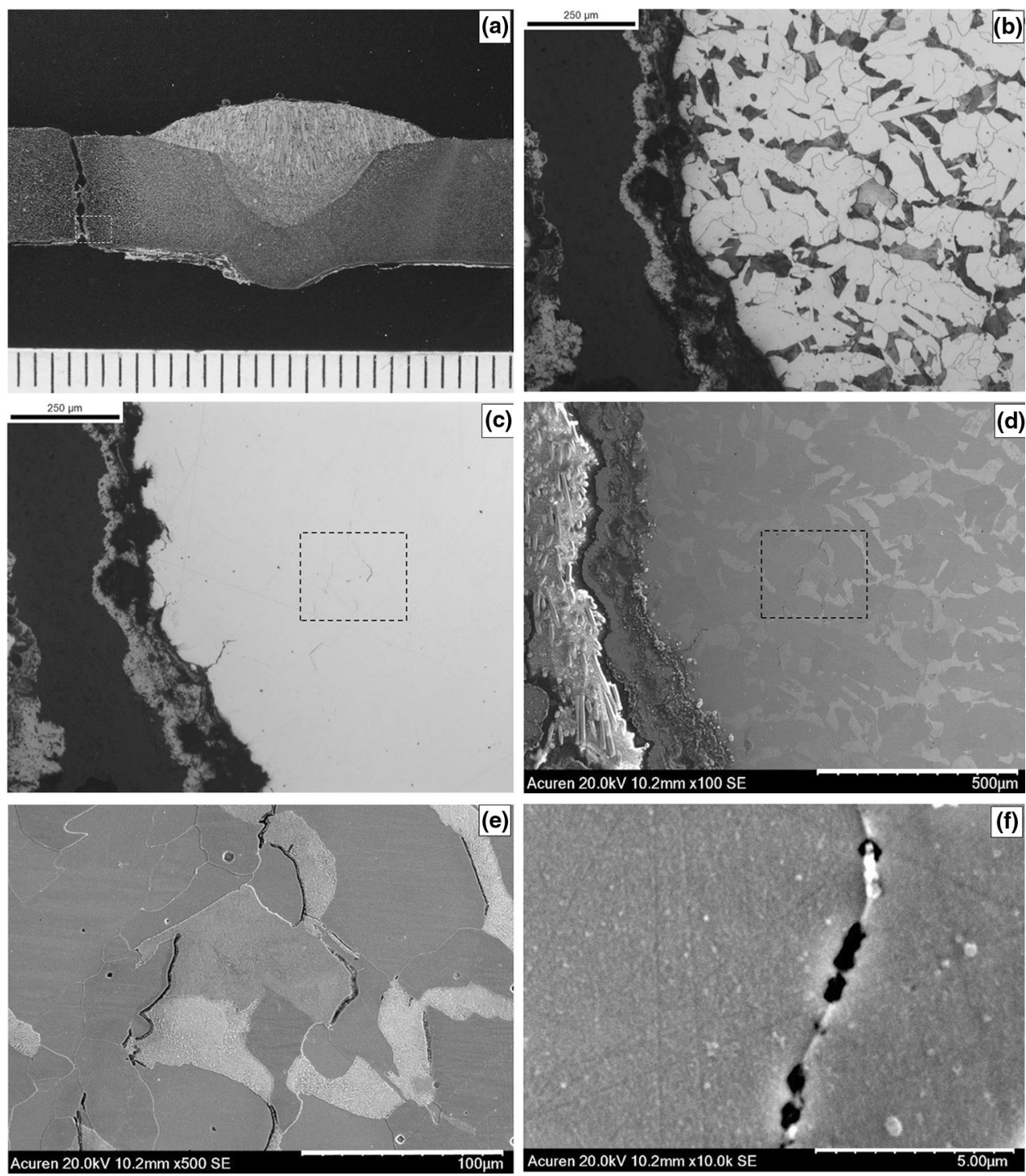

Figure 7 Metallographic examination results: (a) weld macro-section; (b) as-etched optical micrograph of an area adjacent to the main crack and close to the inside surface, $100 \times$; (c) the same area as in 'b' as polished; (d) the same area as in 'b' as viewed in SEM; (e) SEM image of internal fissures near the main cracks, $500 \times$; (f) grain-boundary cavitation, $10,000 \times$

below the main curve and as such it was considered to be in the safe zone. This is in agreement with the results of an inspection performed on the piping 4 years prior to the failure that revealed no cracking. The upset condition, however, was above the long-term exposure carbon steel curve, but below the curve for $10,000 \mathrm{~h}$ (417 days) exposure. This means that, based on these incubation curves, detectable HTHA damage should not have occurred after an upset period of only 275 days at $750{ }^{\circ} \mathrm{F}$ and 116 psia $P_{\mathrm{H} 2}$.

The hydrogen partial pressure limit at $750^{\circ} \mathrm{F}$ can be plotted versus exposure period, as shown in Fig. 9. Interpolation of the data in Fig. 9 for a duration of 6600 h (i.e., 275 days) of upset condition gives a hydrogen partial pressure limit of approximately 191 psia. This means that if the component was in service at an upset condition of $750{ }^{\circ} \mathrm{F}$ and 191 psia $P_{\mathrm{H} 2}$, detectable HTHA damage would likely occur after 275 days. However, HTHA damage occurred at a lower hydrogen pressure of 116 psia. The discrepancy may be due to the contributing factors in the failure, particularly lack of PWHT.
As per the eighth edition of API RP 941 (2016), the incubation curves for carbon steel are for non-welded or PWHT'ed equipment only and no incubation curves are available for welded carbon steel. As a simplified solution, one could shift all the incubation curves $50 \mathrm{psia}$ to the left and $50{ }^{\circ} \mathrm{F}$ down, i.e., the shift made by API RP 941-2016 to the long-term carbon steel curve. This would place the upset condition between the 10,000 and $1000 \mathrm{~h}$ incubation curves (Fig. 10), meaning that the upset condition would be unsafe for $10,000 \mathrm{~h}$ exposure but safe for $1000 \mathrm{~h}$ condition, consistent with the observed HTHA damage after $6600 \mathrm{~h}$ exposure. Interpolation of the data for a duration of $6600 \mathrm{~h}$ on a graph of shifted incubation time versus pressure at $750{ }^{\circ} \mathrm{F}$ (not presented here, but similar to that in Fig. 9) gives a hydrogen partial pressure limit of approximately $126 \mathrm{psia}$, which is close to the upset condition of 116 psia.

It can be concluded from this case study that the new curve for non-PWHT'ed carbon steel appeared too conservative for the inlet piping, as it would suggest that the normal condition 


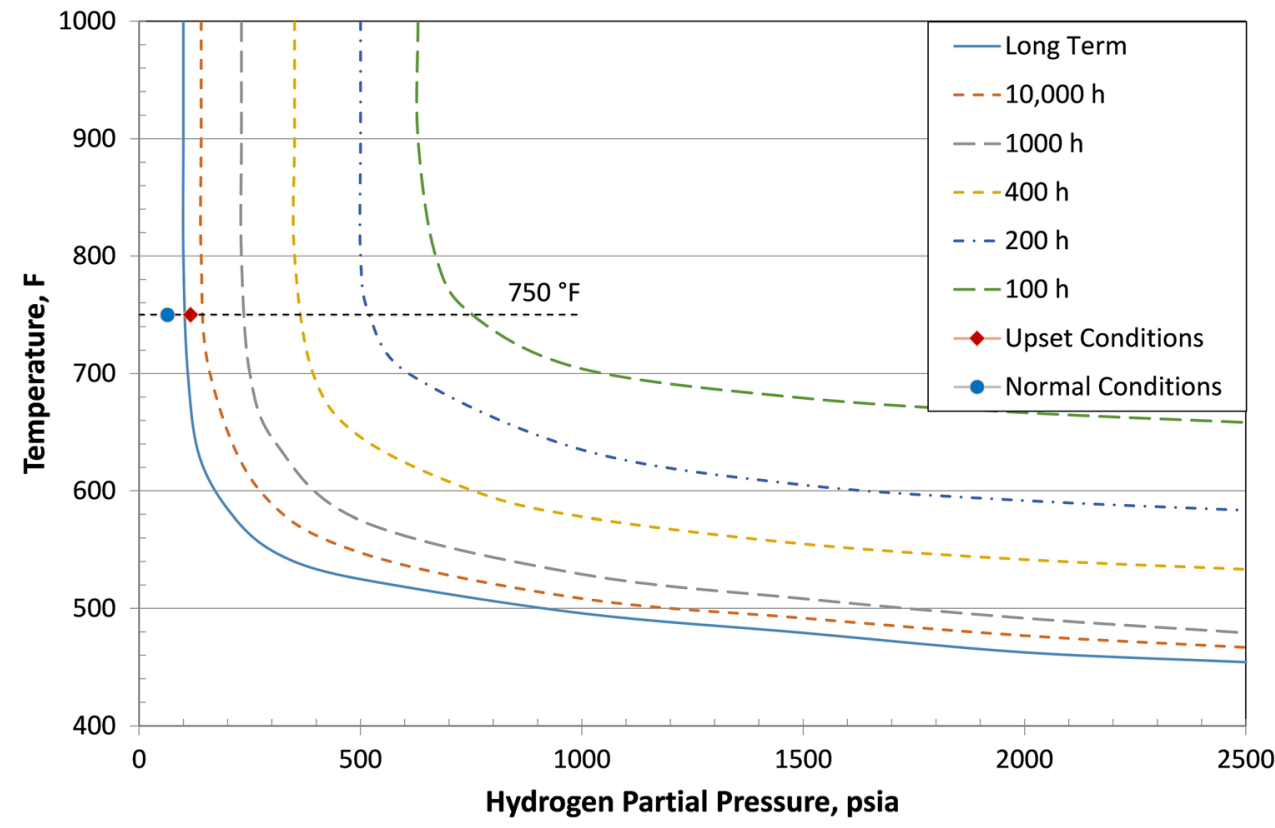

Figure 8 Incubation curves for carbon steel (not welded, or welded and PWHT'ed) (Ref 2); redrawn from the original graph

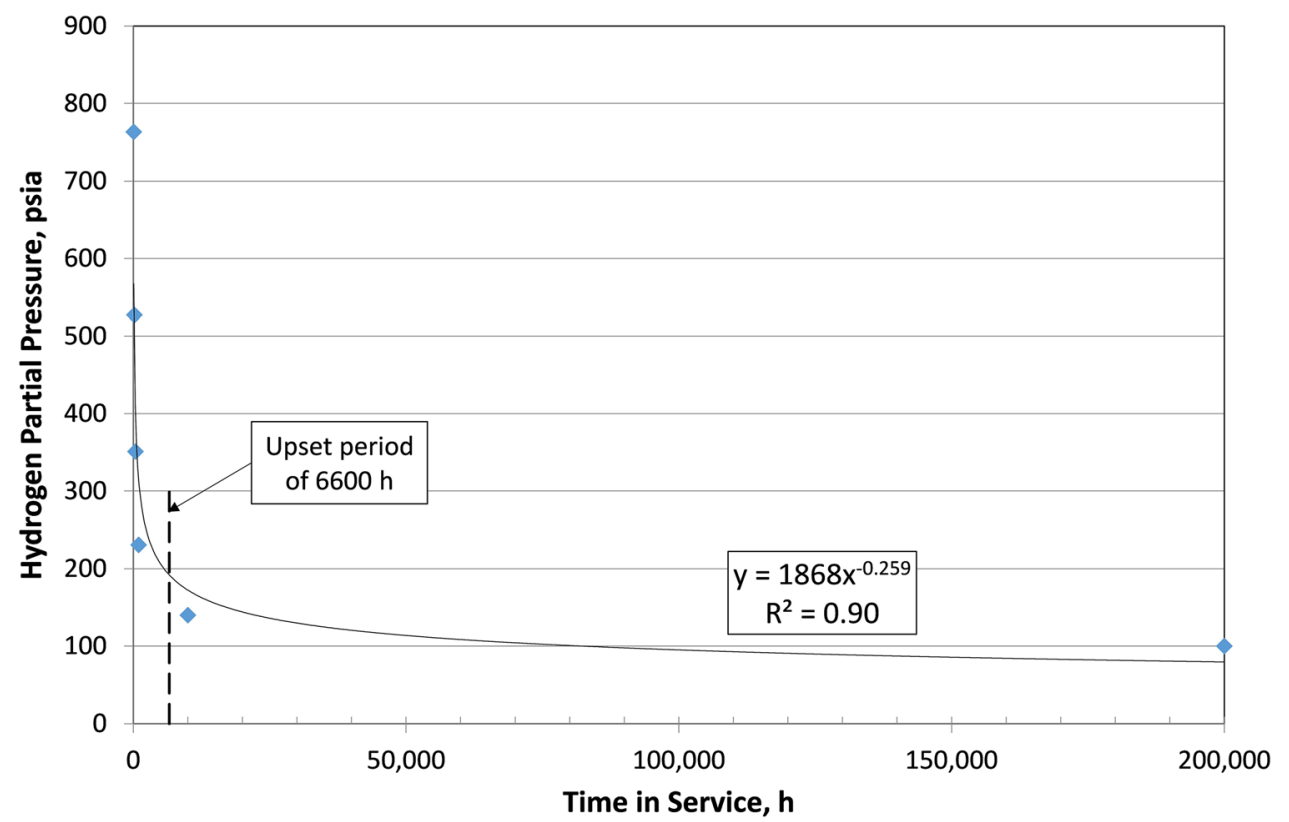

Figure 9 Hydrogen partial pressure limit vs. time in service for carbon steel (not welded, or welded and PWHT'ed) at $750{ }^{\circ} \mathrm{F}$

was unsafe for long-term exposure (e.g., over 200,000 h or 23 years). Therefore, in terms of long-term exposure, the pre2016 curve appeared more applicable by showing that the normal condition would be safe, but the upset condition would be unsafe. The pre-2016 incubation curves, on the other hand, could not predict the HTHA damage at the upset condition. Shifted incubation curves to lower temperature and pressure, however, predicted the HTHA damage during the upset period nicely, in support of the 2016 distinction that the available incubation curves are not to be used for non-PWHT'ed carbon steel. These two contradictory observations regarding the 2016 changes to API RP 941 curves may come from the fact that the incubation curves are generally less accurate for predictions than the long-term curves and may need to be re-established in the future more accurately. The case study was also a reminder that an assessment of the points of high stress on all equipment (i.e., the extrados of the flanged end in this case) is necessary for strategic and successful inspection planning. 


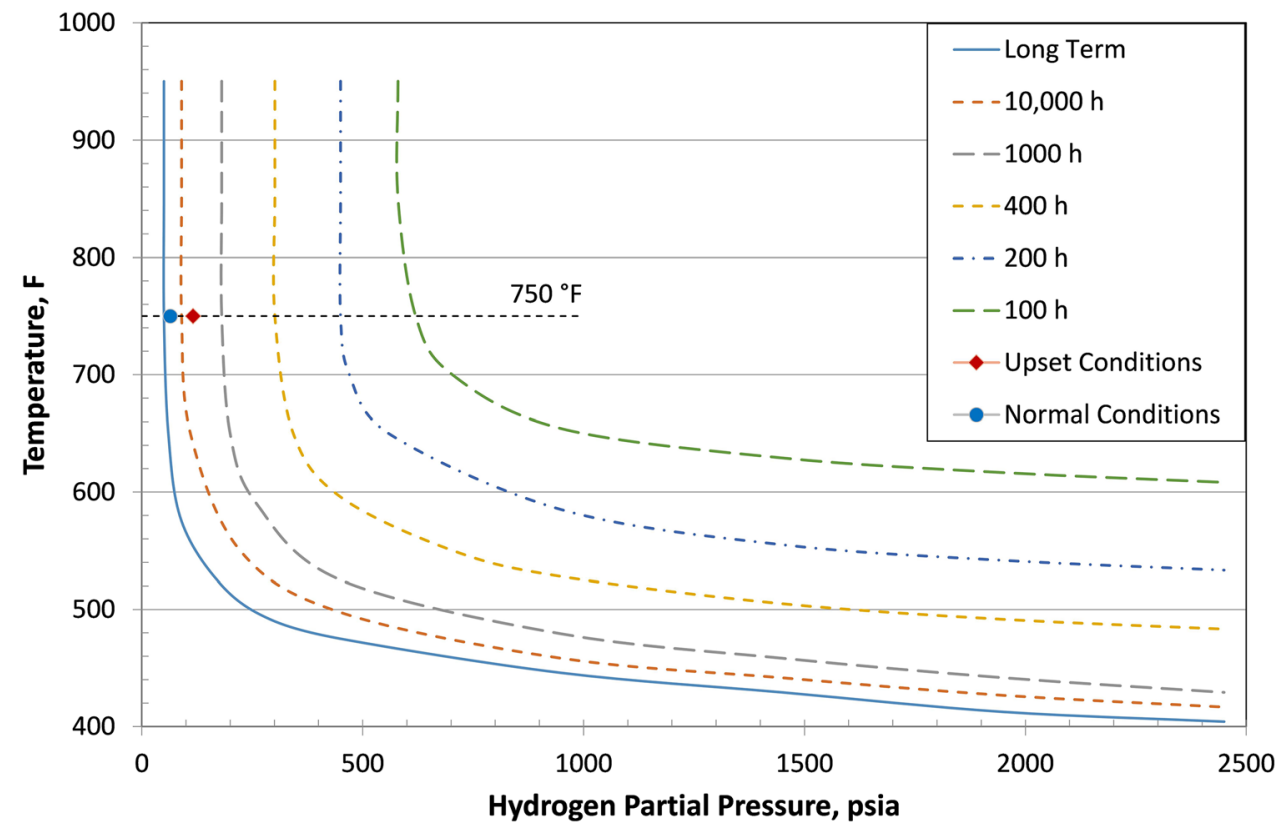

Figure 10 Shifted incubation curves for non-PWHT'ed carbon steel

\section{Future Research Need and Challenges}

The two main areas of research and development in HTHA should be NDE inspection and prediction. From the review of NDE papers in the past few decades, it appears that the industry is on the right path and with the emerging technological development there should be significant progress in detection and sizing of damage at Stage 2 of HTHA or, possibly, at the transition between Stage 1 (incubation) and Stage 2 (rapid attack). The detection of microscopic grain-boundary bubbles close to the inside surface of a vessel (i.e., within Stage 1), however, will most likely remain unachievable by NDE.

Many attempts have been made in the past six decades in developing theoretical models that take into account parameters not considered directly by the Nelson curves, particularly time and stress. Unfortunately, no single model has yet emerged to be accepted globally for the prediction of HTHA failures. What industry likely needs is the continuation in development of simple prediction tools, such as reliable boundary conditions and incubation curves (with clear criteria for attack) and/or HAT charts (including critical values of $P_{\mathrm{V}}$ or $P_{\mathrm{W}}$ for different conditions) for the main generic steel types and in different conditions (i.e., with and without PWHT for carbon steel). This could take care of the effect of time in a practical way.

Although the effect of stress is not addressed in the Nelson curves explicitly and no $T-P$ contours (similar to incubation curves) are available for the effect of stress, "normal" service stress is inherently included in the Nelson curves. The curves are established primarily based on the actual industrial data and all equipment in service are subjected to external stress (i.e., internal-pressure-induced hoop and axial stresses as well as bending stresses at the flanges that fluctuate upon heating and cooling cycles). Conceivably, the reported HTHA damage (or at least failures) had occurred at the areas of high stress. Therefore, as long as the magnitude of stress is not unusually high on a component, the Nelson curves (or Nelson-type curves) should remain to be a reasonable predictive tool for
HTHA failures. The results of the case study presented in Section 20 is a testimony to this opinion. The case study showed that although the transverse crack occurred on one side (i.e., the extrados), indicative of a significant bending stress, the pre-2016 Nelson curve for carbon steel predicted the long-term HTHA response of the spool correctly. There was a discrepancy when using the incubation curves, but that can be rectified by establishing more accurate incubation curves (for both PWHT'ed and non-PWHT'ed conditions) with consistent criteria for attack.

Nevertheless, it would be beneficial to have some laboratory-based $T-P$ contours for each generic material group at several levels of external stress, e.g., $0,25,50$ and $75 \%$ of the nominal yield strength. Interpolation of the data derived from plotting a variable of interest (e.g., $t, T$ or $P$ ) against the external stress level can predict the possibility of HTHA damage under a specific level of stress determined for a location of high-stress on a component. Stress estimation may be done through analytical or numerical calculations. High-temperature strain gauges may also be used during start-up heating to assess thermally induced stresses on the components.

\section{Conclusions}

The following conclusions can be drawn from the historical review of HTHA investigations between 1930 and 2020:

1. HTHA was discovered in the early twentieth century in Germany. Our understanding of the damage mechanism(s) and our knowledge on its different aspects grew decade by decade. Distribution of the published articles on the subject since 1930 reveals several peaks, some of which were shortly after catastrophes, namely WWII and the deadly plant explosions in Japan (1982) and USA (2010), and the subsequent changes to regulations 
and recommended practices (especially API RP 941 in 1990 and 2016).

2. In terms of terminology, hydrogen attack was the prevalent term in the twentieth century referring to the hightemperature, irreversible hydrogen damage. In the twenty first century, high-temperature hydrogen attack (with the acronym HTHA) became the predominant term, after going through some transitional terms such as hot hydrogen attack, to distinguish it from the lowtemperature, reversible hydrogen embrittlement.

3. HTHA can result in bubble nucleation at inclusions, carbides or grain-boundaries (that may or may not have second phases). The chemical reaction between atomic hydrogen and solutes, carbides or inclusions can result in the formation of non-soluble gasses such as methane, molecular hydrogen, hydrogen sulphide, steam or ammonia in the cavities. Methane formation is the main chemical reaction in HTHA of steel.

4. HTHA occurs in carbon steel in the temperature range 200-600 ${ }^{\circ} \mathrm{C}$, with $0.7 \mathrm{MPa}$ (100 psia) hydrogen partial pressure minimum. Many factors can affect the susceptibility temperature range and the rate of damage, such as (residual or applied) stress, (actual as opposed to nominal) composition and prior thermal history (i.e., heat treatment or welding).

5. HTHA resistance depends on the activity of carbon (and, to a lesser degree, other solutes such as sulphur and oxygen), which is a function of matrix solubility for carbon, carbide stability and carbon/carbide concentration. Other factors include grain-boundary condition (i.e., surface energy and solute segregation concentration that may have a retarding effect on carbon diffusion along the grain boundary or may conceivably affect decohesion strength at the grain boundary), surface condition (where hydrogen adsorption, dissociation and ingress occurs) and the matrix creep strength (affecting the methane bubble growth rate).

6. HTHA is described to have two main forms, namely surface decarburization (without fissuring) and internal decarburization along with fissuring. In reality, only the latter is due to HTHA and resulting in the entrapment of non-soluble gasses in the metal. The former occurs by carbon diffusion to the surface, is driven by carbon concentration gradient, occurs mostly at temperatures higher than methane formation temperature limit (i.e., approximately $600{ }^{\circ} \mathrm{C}$ ) and does not require hydrogen ingress into the metal. Surface decarburization can also take place in oxidizing environments.

7. The recent distinction of non-conventional or non-classical HTHA (based on damage morphology, damage location, prior thermal history (particularly PWHT) and service conditions) may not be necessary and may need careful reconsideration. HTHA may manifest itself somewhat differently under different conditions (e.g., based on the magnitude of applied stress) or at different weld/HAZ sub-regions.

8. The three main stages of attack (based on mechanical property change) are incubation, rapid attack and saturation (or steady state). There have been many other categorizations based on different aspects of the damage such as chemical reaction and kinetics steps. The division of the incubation period to detectable and non-detectable in the seventh and the eighth editions of API
RP 941 with evolving descriptions may require revaluation especially in terms of the provided descriptions that appear problematic, as discussed in Section 9.2.

9. It cannot be determined which weld region (i.e., weld metal or different HAZ sub-regions) is most susceptible to HTHA. Many factors (such as stress, composition and thermal history) affect the area of highest susceptibility. There have been several reports of failure of piping weldments just outside the HAZ, i.e., in the subcritical HAZ, that has not been studied well either experimentally or theoretically.

10. Detection and sizing of HTHA damage in its early stage of growth (i.e., prior to the formation of macro-cracks) by NDE is a key field of research that is still under development and progress. There are several UT techniques that have shown promise in the detection of HTHA damage at relatively early stages of attack, examples of which include AUBT, TOFD and PAUT with or without total focusing method. The degree of success of a method/technique is not merely an inherent factor and it depends largely on the procedural details (such as selection of parameters) and the skill of the technician who executes the test.

11. All HTHA lab tests should be compared with a test in argon in order to separate HTHA from metallurgical ageing. In addition, the samples need to be degassed by low-temperature annealing (e.g., in vacuum at $250{ }^{\circ} \mathrm{C}$ for $3 \mathrm{~h}$ ) after the exposure to hydrogen at the elevated temperature and prior to mechanical testing to remove diffusible hydrogen and separate HTHA from HE.

12. Very high-temperature annealing, e.g., isothermally at $680-900{ }^{\circ} \mathrm{C}$ for several hours or cyclically between the room temperature and $1000{ }^{\circ} \mathrm{C}$ with a total time of 10 $\mathrm{h}$, has been proposed for healing of microscopic HTHA damage and recovery of (some) properties in the past. However, the associated changes in the microstructure (due to austenitization and grain growth) and mechanical properties raise doubt on the practicality/usefulness of such procedures.

13. The Nelson curve for $0.5 \mathrm{Mo}$ steel was removed from API Publication 941 in 1990 after receiving many failure reports of $0.5 \mathrm{Mo}$ steel equipment under the Nelson curve (i.e., in the safe zone). The problem with this type of material appears to be due to the effects of heat treatment and actual composition on the carbide type and stability. Rigorous inspection should be in place for $0.5 \mathrm{Mo}$ steel equipment still in service.

14. API RP 941 added a second carbon steel curve in 2016 for welded, but not PWHT'ed, carbon steel, which is approximately $50 \mathrm{psi}$ to the left of the preceding carbon steel curve at the vertical leg and approximately $50{ }^{\circ} \mathrm{F}$ lower at the horizontal leg. This change places many carbon steel equipment already in service in, or very close to, the "unsafe zone". Future reports from the end-users will show if such conservative regulation was necessary or the actual problem resulting in the unexpected failures of carbon steel piping has been excursion beyond the nominal operating conditions and/or improper shutdowns and start-ups.

15. Nelson curves do not take into account the effect of time. This deficiency has been addressed by establishing incubation curves (for carbon steel) or developing HAT charts for $0.5 \mathrm{Mo}$ and $\mathrm{Cr}-\mathrm{Mo}$ steels. HAT charts and the 
use of time-included HTHA parameters (i.e., $P_{\mathrm{V}}$ and $\left.P_{\mathrm{W}}\right)$, as developed in Japan in the 1990 s, does not appear to be appreciated in the rest of the world as much. The parameter $P_{\mathrm{V}}$ had been implemented in API RP 581 to assess HTHA susceptibility, but it was removed from its third edition in 2016.

16. Many HTHA models have been developed in the last six decades. The growth models typically take into account an interaction between methane pressure, powerlaw creep and grain-boundary diffusion. Models are used in FFS evaluation, remaining life assessment and inspection planning.

\section{Acknowledgements}

The author wishes to thank the University of Alberta Library for providing access to over 220 articles used in this review paper. The author also wishes to thank Acuren Group Inc. (for which the author works in the capacity of an Engineering Technical Advisor in Edmonton) for providing professional development opportunity to work on the paper and the use of its lab for the failure analysis case study. In particular, the author is thankful to Mr. Dale Harsulla (P.Eng., Engineering and Lab Advisor, Acuren, Edmonton) for peer-reviewing the article prior to submission to the journal. The author would also like to thank the journal reviewers for their time and comments that have made this article more presentable.

\section{References}

1. Catastrophic Rupture of Heat Exchanger, U.S. Chemical Safety and Hazard Investigation Board, 2014

2. Steels for Hydrogen Service at Elevated Temperatures and Pressures in Petroleum Refineries and Petrochemical Plants, API RP 941, 8th edn., American Petroleum Institute, 2016

3. M. Smialowski, Chapter 18: Action of Hydrogen on Steel under High Pressure, in Hydrogen in Steel: Effect of Hydrogen on Iron and Steel during Production, Fabrication and Use, Pergamon Press, Oxford, pp. 411-420, 1962

4. E.E. Fletcher and A.R. Elsea, The Effects of High-Pressure HighTemperature Hydrogen on Steel, Defense Metals Information Center, Columbus, Ohio, 1964

5. W. Beck, E. Jankowsky and P. Fischer, Chapter 10: Damaging Effects of High Pressure Hydrogen at Elevated and Ambient Temperatures, in Hydrogen Stress Cracking of High Strength Steel-Report No. NADC-MA-7140, Washington, D.C., Department of the Navy, 1971, pp. $114-122$

6. P.G. Shewmon, Hydrogen Attack of Pressure-Vessel Steels, Mater. Sci. Technol., 1985, 1(1), p 2-11

7. C. Nageswaran, Maintaining the Integrity of Process Plant Susceptible to High Temperature Hydrogen Attack. Part 1: Analysis of Nondestructive Testing Techniques, Health and Safety Executive, 2018

8. J. Rothwell, Maintaining the Integrity of Process Plant Susceptible to High Temperature Hudrogen Attack. Part 2: Factors Affecting Carbon Steel, Health and Safety Executive, 2018

9. High Temperature Hydrogen Attack, in Damage Mechanisms Affecting Fixed Equipment in the Refining Industry-API Recommended Practice 571, 3rd edn., American Petroleum Institute, 2020, pp. 189 196

10. C.A. Zapffe, Boiler Embrittlement, Trans. ASME, 1944, 66, p 81-126

11. Various, Discussion on Boiler Embrittlement by C. A. Zapffe, Trans. ASME, 1944, 66, p. 117

12. R. Effinger, M. Renquist, A. Wachter and J. Wilson, Hydrogen Attack of Steel in Refinery Equipment, in American Petroleum Institute-Proceedings, 1951
13. W.H. Johnson, On Some Remarkable Changes Produced in Iron and Steel by the Action of Hydrogen and Acids, Proc. R. Soc. Lond., 1875, 23, p 168-179

14. F.K. Naumann, Der Einfluss von Legierungszusaetzen auf die Bestaendigkeit von Stahl gegen Wasserstoff unter hohem Druck (Influence of Alloying Elements on Resistance of Steel to Attack of Hydrogen under High Pressure), Stahl Eisen, 1938, 58(44), p 12391249

15. Anonymous, Hydrogen Attack of Steel, in Metallurgist-Supplement to Engineer, Feb 25, pp. 108-110, 1938

16. J. Schuyten, Hydrogen Attack on Metals at High Temperatures and Pressures, in Corrosion and Material Protection, vol. 4, no. 5; SeptOct, pp. 13-18, 1947

17. G. Nelson, Hydrogenation Plant Steels, in American Petroleum Institute-Proceedings, vol. 29m, no. Sec 3, pp. 163-172, 1949

18. H.K. Ihrig, Attack of Hydrogen-Nitrogen Mixtures on Steels, Ind. Eng. Chem., 1949, 41(11), p 2516-2521

19. L. Weiner, Kinetics and Mechanism of Hydrogen Attack of Steel, Corrosion, 1961, 17(3), p 137t-143t

20. H.H. Podgurski, Trapping of Hydrogen in Cold-Worked Steel, Trans. AIME, 1961, 221, p 389-394

21. R.E. Allen, R.J. Jansen, P.C. Rosenthal and F.H. Vitovec, The Rate of Irreversible Hydrogen Attack of Steel at Elevated Temperatures, in American Petroleum Institute-Proceedings, 1961

22. C. Lundin, M. Bharadwaj and M. Prager, Progress in Assessing the Safety of C-1/2 Mo Equipment in Long Term Elevated Temperature Hydrogen Pressure Vessel and Piping Service, in WRC Bulletin, Current Concepts for Life Assessment of Pressure Vessels and Piping, vol. 568 Shaker Heights, OH, Welding Research Council Inc., pp. 109-134, 2016

23. F. Worzala and D. Westphal, The Incubation Stage of Hydrogen Attack, Scr. Metall., 1971, 5, p 43-48

24. H. Shih and H. Johnson, Inclusions, Grain Boundaries and Hydrogen Attack, Scr. Metall., 1977, 11, p 151-154

25. A. Sagues, B.O. Hall and H. Wiedersich, On the Mechanism of Hydrogen Attack, Scr. Metall., 1978, 12, p 319-326

26. Y. Hasegawa, Failures from Hydrogen Attack and Their Methods of Detection, Weld. Int., 1988, 2(6), p 514-521

27. H. Shih and H. Johnson, A Model Calculation of the Nelson Curves for Hydrogen Attack, Acta Metall., 1982, 30, p 537-545

28. Z. Su, D. Stone, J. Wanagel and C.-Y. Li, Hydrogen Attack in a Low Si-0.10C-2-1/4Cr-1Mo-1/4V-Ti-B Steel, Mater. Sci. Eng., 1984, 62, p 261-264

29. T. Watanabe, Y. Hasegawa and K. Kato, Ultrasonic Velocity Ratio Method for Detecting and Evaluating Hydrogen Attack in Steels, in Corrosion Monitoring in Industrial Plants Using Nondestructive Testing and Electrochemical Methods, Philadelphia, 1986

30. K. Yokogawa, S. Fukuyama, K. Kudo and P.G. Shewmon, Effect of Hydrogen Attack on Tensile and Creep Properties of Low Carbon Steel, Int. J. Pres. Ves. Piping, 1989, 37, p 365-385

31. E. Lunarska and Z. Szklarska-Smialowska, Hydrogen Assisted Fracture of Inconel 600 Charged at High Temperature, in Proceedings of The 7th International Conference On Fracture (ICF7), Houston, Texas, 1989

32. A. Birring, M. Bartlett and K. Kawano, Ultrasonic Detection of Hydrogen Attack in Steels, Corrosion, 1989, 45(3), p 259-263

33. K. Hattori and S. Aikawa, Scheduling and Planning Inspection of C1/2 Mo Equipment Using the New Hydrogen Attac Tendency Chart, in American Society of Mechanical Engineers, Pressure Vessels and Piping Division (Publication) PVP, vol. 239, pp. 121-128, 1992

34. M.W.D. van der Burg, E. van der Giessen and V. Tvergaard, Numerical study of Hydrogen Attack in Creeping Vessels, in Computer Aided Assessment and Control of Localized Damage-Proceedings of the International Conference, 1996

35. M. Van der Burg and E. Van der Giessen, A Continuum Damage Relation for Hydrogen Attack Cavitation, Acta Mater., 1997, 45(7), p 3047-3057

36. M. Van der Burg, E. Van der Giessen and V. Tvergaard, A Continuum Damage Analysis of Hydrogen Attack in a 2.25Cr-1Mo Pressure Vessel, Mater. Sci. Eng. A, 1998, 241, p 1-13

37. G.A. Nelson, Hydrogenation Plant Steels, in American Petroleum Institute-Proceedings, vol. 29m, no. Sec 3, pp. 163-172, 1949 
38. M. Prager, A Worldwide Look at Hot Hydrogen Attack, in American Society of Mechanical Engineers, Pressure Vessels and Piping Division (Publication) PVP, vol. 411, 2000

39. "CSB Safety Alert: Preventing High Temper-ature Hydrogen Attack (HTHA), August 2016. Available: http://www.csb.gov/assets/1/20/sa fety_alert_htha_final.pdf?15689

40. L.C. Weiner, On the Kinetics of Irreversible Hydrogen Embrittlement, Acta Metal., 1960, 8, p 52-53

41. W.T. Becker and R.J. Shipley, eds., Hydrogen Damage and Embrittlement, in ASM Handbook, Failure Analysis and Prevention, vol. 11, ASM International, 2002, pp. 809-822

42. Steels for Hydrogen Service at Elevated Temperatures and Pressures in Petroleum Refineries and Petrochemical Plants, API Publication 941, 2nd edn., American Petroleum Institute, 1977

43. R.T. Effinger, M.L. Renquist, A.G. Wachter and J.G. Wilson, Hydrogen Attack of Steel in Refinery Equipment, in American Petroleum Institute-Proceedings, 1951

44. W.A. Bonner, H.D. Burnham, J.J. Conradi and T. Skei, Industry Probes Means of Combating Hydrogen Attack on Steel in Refinery Equipment, Pet. Process., 1953, 8(6), p 878-883

45. A. Sagues, Dilatometer for the In Situ Observation of HighTemperature, High-Pressure Hydrogen Attack, Rev. Sci. Instrum., 1979, 50(1), p 48-51

46. O.-T. Saugerud, D.E. Moore and G. Odette, Model-Based Approach to Evaluating the Probability and Critically of Hot Hydrogen Attack in C- 1/2 Mo Equipment, in American Society of Mechanical Engineers, Pressure Vessels and Piping Division (Publication) PVP, vol. 239,1992

47. J.E. Cantwell, High Temperature Hydrogen Attack: A 50 Year Overview, in Corrosion 93, The NACE Annual Conference and Corrosion Show, Paper No. 538, Houston. TX, 1993

48. A. Kermad, R. Browne, K. Narayansingh and D. Bigford, Hydrogen Attack of $1 \mathrm{Cr} 1 / 2$ Mo Catalytic Reforming Reactors, in American Society of Mechanical Engineers, Pressure Vessels and Piping Division (Publication) PVP, vol. 336, 1996

49. C. Panzarella and J. Cochran, The E2G Model of High Temperature Hydrogen Attack and the New Prager Curves, in WRC Bulletin Current Concepts for Life Assessment of Pressure Vessels and Piping, vol. 568, Shaker Heights, OH, Welding Research Council Inc., pp. 135-169, 2016

50. M.J. Yacamán, T.A. Parthasarathy and J.P. Hirth, Hydrogen Attack in an Austenitic Stainless Steel, Metall. Trans. A, 1984, 15(7), p 14851490

51. X. Li, H. Chen, Z. Yao, J. Li and W. Ke, Hydrogen Attack on Austenitic Steel 304 under High Temperature and High Pressure, Acta Metall. Sinica Ser. B, 1993, 6(5), p 374-378

52. C.-M. Suh, S.-H. Nahm, J.-H. Kim and Y.-S. Pyun, A Study on the VHCF Fatigue Behaviors of Hydrogen Attacked Inconel 718 Alloy, Trans. Korean Soc. Mech. Eng. A, 2016, 40(7), p 637-646

53. R.E. Allen, R.J. Jansen, P.C. Rosenthal and F.H. Vitovec, Analysis of Probable Mechanisms of High-Temperature Hydrogen Attack of Steel, in American Petroleum Institute-Proceedings, vol. 42, no. Sec 3, pp. 452-462, 1962

54. J. Krynicki, K. Bagnoli and J. McLaughlin, Probabilistic Risk Based Aprroach for Performing an Onstream High Temperature Hydrogen Attack Inspection, in 61st Annual Conference \& Exposition, 2006

55. R. Bisaro and G. Geiger, Effects of Columbium in Steel on Elevated Temperature Hydrogen Attack, Corrosion, 1967, 23(10), p 289-296

56. S. Nomura and M. Hasegawa, Effect of Cementite Distribution in Low Carbon Steel on Hydrogen Attack, Tetsu-To-Hagane/J. Iron Steel Inst. Jpn., 1975, 61(11), p 2579-2588

57. M. Menyhard and G. Zolnay, Direct Measurement of the Gas Content of Blisters Formed by Hydrogen Attack during Steam Corrosion in a Low Alloy Steel, Metall. Trans. A, 1983, 14(10), p 2187-2189

58. M. Van der Burg and E. Van der Giessen, Non-Uniform Hydrogen Attack Cavitation and the Role of Interaction with Creep, Mater. Sci. Eng. A, 1996, 220, p 200-214

59. G. Odette and S. Vagarali, An Equation-of-State for Methane for Modeling Hydrogen Attack in Ferritic Steels, Metall. Trans. A, 1982 , 13(2), p 299-303

60. T.A. Parthasarathy, H.F. Lopez and P.G. Shewmon, Hydrogen Attack kinetics of 2.25 Cr-1 Mo steel weld metals, Metall. Trans. A, 1985, 16(6), p 1143-1149
61. G. Sundararajan and P. Shewmon, The Hydrogen Attack of HSLA Steels, Metall. Trans. A, 1980, 11A(3), p 509-516

62. A.R. Ciuffreda and W.D. Rowland, Hydrogen Attack of Steel in Reformer Service, in American Petroleum Institute-Proceedings, 1957

63. F.H. Vitovec, Growth Rate of Fissures during Hydrogen Attack of Steels, in American Petroleum Institute-Proceedings, 1964

64. S. Vagarali and G. Odette, A Model for the Growth of Hydrogen Attack Cavities in Carbon Steels, Metall. Trans. A, 1981, 12(12), p 2071-2082

65. F. Vitovec, Modeling of hydrogen attack of steel in relations to material and environmental variables, in Current Solutions to Hydrogen Problems in Steels, American Society for Metals, 1982, pp. 236-241

66. M. Al-Arada and M. Al-Otaibi, Evaluation of High Temperature Hydrogen Attack Effect on Carbon Steel-0.5 Mo Heat Exchanger, in Corrosion 2014, San Antonio, TX, 2014

67. M. Wahab, B. Saba and A. Raman, Fracture Mechanics Evaluation of a $0.5 \mathrm{Mo}$ Carbon Steel Subjected to High Temperatute Hydrogen Attack, J. Mater. Process. Inf. Technol., 2004, 153-154, p 938-944

68. J. McLaughlin, J. Krynicki and T. Bruno, Cracking of Non-PWHT'd Carbon Steel Operating at Conditions Immediately Below the Nelson Curve, in Proceedings of ASME 2010 Pressure Vessels \& Piping Division/K-PVP Conference, Bellevue, Washington, 2010

69. T Silfies, J Dobis and M Nugent, Looking Behind the Curtain of API RP 941 High Temperature Hydrogen Attack (HTHA) Data, in Corrosion, 2016 Conference \& Expo Paper No. 7516, 2016

70. Y. Honma and K. Hashi, Effect of Residual Stress on High Temperature Hydrogen Attack for Pressure Vessels, in ASME 2019 Pressure Vessels and Piping Conference, PVP2019-94058, 2019

71. K. Poorhaydari, Failure of a Hydrogenerator Reactor Inlet Piping by High-Temperature Hydrogen Attack, Eng. Fail. Anal., 2019, 105, p 321-336

72. G.A. Nelson and R.T. Effinger, Blistering and Embrittlement of Pressure Vessel Steel by Hydrogen, Weld. J., 1955, 34(1), p 12s-21s

73. A. Sagues, Enhancement of Hydrogen Attack by the Presence of Implanted Helium, J. Nuclear Mater., 1979, 79, p 260-263

74. Risk-Based Inspection Methodology, API RP 581, 3rd edn, American Petroleum Institute, 2016

75. T. Parthasarathy, Mechanisms of Hydrogen Attack of Carbon and 21/4Cr-1Mo Steels, Acta Metal., 1985, 33(9), p 1673-1681

76. J.J.R. Thygeson and M.C. Molstad, High Pressure Hydrogen Attack of Steel, J. Chem. Eng. Data, 1964, 9(2), p 309-315

77. L. Looney, R. Hurst and D. Taylor, The Effect of High Pressure Hydrogen on the Creep Fracture of Notched Ferritic Steel Components, J. Mater. Process. Technol., 1998, 77, p 25-31

78. G.A. Nelson, Use Curves to Predict Steel Life, Hydrocarbon Process. Pet. Refiner, 1965, 44(5), p 185-188

79. C Woods and T Scott, Hydrogen Attack of Bainitic 2 1/4 Cr-1 Mo Steel, in Microstructural Science Fifteenth Annual Technical Meeting of the International Metallographic Society, Orlando, FL, USA, 1983

80. T. Sakai and K. Asami, Effect of Applied Stress on Hydrogen Attack of 2 1/4 Cr-1Mo Steel, Tetsu-To-Hagane/J. Iron Steel Inst. Jpn., 1987, 73(3), p 551-557

81. T. Sakai, T. Nomura and E.H. Niccolls, The basis and application of the "Pw" parameter for high temperature hydrogen attack, in American Society of Mechanical Engineers, Pressure Vessels and Piping Division (Publication) PVP, vol. 411, 2000

82. C. Allevato, Utilizing Acoustic Emmision Testing to Detect HighTemperature Hydrogen Attack (HTHA) in Cr-Mo Reformer Reactors and Piping during Thermal Gradients, Procedia Eng., 2011, 10, p 3552-3560

83. S. Ruoff, D. Stone and C.-Y. Li, Hydrogen Attack in $3 \mathrm{Cr}-1.5 \mathrm{Mo}$ Steel at Elevated Temperature, Mater. Sci. Eng., 1987, 93, p 217-225

84. M. Van der Burg, E. Van der Giessen and R. Brouwer, Investigation of Hydrogen Attack in $2.25 \mathrm{Cr}$-1Mo Steels with a High-Traxiality Void Growth Model, Acta Mater, 1996, 44(2), p 505-518

85. T. Imanaka and J.I. Shimomura, Temper Embrittlement and Hydrogen Attack on 2 1/4 Cr-1Mo Steels in High Pressure and High Temperature Hydrogen Atmosphers, in 5th International Conference on Pressure Vessel Technology, 1984

86. T. Imanaka, J.-I. Shimomura, S. Nakano and K. Yasuda, Hydrogen Attack in Cr-Mo Steels and Disbonding of Austenitic Stainless Weld Overlay, Kawasaki Steel Tech. Rep., 1985, 13, p 109-119 
87. J. Shimomura and T. Imanaka, Decomposition of Carbides in 2-1/ 4Cr-1Mo Steels during Hydrogen Attack, Scr. Metall., 1985, 19, p $1507-1511$

88. T. Sakai and H. Kaji, Effects of $\mathrm{P}, \mathrm{Sn}, \mathrm{As}, \mathrm{Sb}, \mathrm{Si}$, and $\mathrm{Cu}$ on the Formation of Bubbles by Hydrogen Attack in $2.25 \mathrm{Cr}-1 \mathrm{Mo}$ Steel, Tetsu-To-Hagane/J. Iron Steel Inst. Jpn., 1980, 66(8), p 1133-1141

89. T. Imanaka, S. Sato, K. Aso, J. Shimomura, S. Ueda, A. Ejima and N. Ohashi, Improvement in Elevated Temperature Strength, Hydrogen Attack Resistivity and Stress Relief Cracking Susceptibility of Cr-Mo Steels, Nucl. Eng. Des., 1986, 96, p 195-207

90. M. Nagae, H. Tsukamoto, H. Tagawa, H. Suzuki, M. Hayashida and T. Nagamine, Development of 2 1/4Cr-1Mo Steel with High Strength and High Resistance to Hydrogen Attack, Nippon Kokan Tech. Rep. Overseas, 1987, 51, p 11-19

91. J.-I. Shimomura, H. Tani, T. Kooriyama, S. Sato and S. Ueda, High Strength 2 1/4 and 3\% Cr-1\% Mo Steels with Excellent Hydrogen Attack Resistance, Kawasaki Steel Tech. Rep., 1989, 20, p 78-87

92. T. Ishiguro, K. Ohnishi and J. Watanabe, Effects of $\mathrm{Cr}$ and $\mathrm{V}$ on the Hydrogen Attack Susceptibility of B Added Cr-Mo Steels, Tetsu-ToHagane/J. Iron Steel Inst. Jpn., 1986, 72(1), p 70-77

93. S. Pillot, S. Corre, L.C.C. Coudreuse and P. Toussaint, Development and Production of Creep and Hydrogen Resistant Grade 91 (9Cr1MoV) Heavy Plates for New Generation High Efficiency Refining Reactors, 2013

94. T. Sakai, K. Asami, N. Kondo and T. Hayashi, Effects of Carbide Forming Elements on Hydrogen Attack and Embrittlement in 2 1/4 Cr-1Mo Steel, Tetsu-To-Hagane/J. Iron Steel Inst. Jpn., 1987, 73(2), p 372-379

95. H. Nakajima, H. Miyaji, S. Yamamoto, T. Kasugai and S.-I. Hasegawa, Effect of Carbide Forming Elements on Hydrogen Attack of 2.25Cr-1Mo Steel, Tetsu-To-Hagane/J. Iron Steel Inst. Jpn., 1990, 76(8), p 1372-1379

96. H. Nakajima, H. Miyaji and S. Yamamoto, Effect of Prior-Austenite Grain Size on Hydrogen Attack of 2.25Cr-1Mo Steel, Tetsu-ToHagane/J. Iron Steel Inst. Jpn., 1991, 77(8), p 1320-1327

97. G.A. Nelson, Operating Limits and Incubation Times for Steels in Hydrogen Service, in American Petroleum Institute-Proceedings, 1965

98. R. Chiba, Effect of Carbon on Hydrogen Attack of 2-1/4 Cr-1Mo Steels, Tetsu-To-Hagane/J. Iron Steel Inst. Jpn., 1985, 71(14), p 1639-1646

99. R. Pishko, M. McKimpson and P. Shewmon, The Effect of Steelmaking on the Hydrogen Attack of Carbon Steel, Metall. Trans. A, 1979, 10A(7), p 887-894

100. P.C. Rosenthal, W.L. Schroeder and F.H. Vitovec, Effect of LowAlloy Additions on Resistance of Carbon Steels to Hydrogen Attack, in American Petroleum Institute-Proceedings, 1963

101. T. Sakai, Void Growth by Hydrogen Attack in $21 / 4 \mathrm{Cr}-1 \mathrm{Mo}$ and $3 \mathrm{Cr}-$ 1Mo Steels, R D Res. Dev. Kobe Steel Eng. Rep., 1986, 36(2), p 90 93

102. T. Nomura and T. Sakai, Theoretical and Parametric Methods for Evaluating Hydrogen Attack Limit, in American Society of Mechanical Engineers, Pressure Vessels and Piping Division (Publication) PVP, vol. 359, 1997

103. K. Kimura, T. Ishiguro, K. Kawano, K. Hattori, H. Yamamoto, H Okada and K. Sakamoto, Metallurgical Effect on Hydrogen Attack Damage C-0.5Mo Steels, in American Society of Mechanical Engineers, Pressure Vessels and Piping Division (Publication) PVP, vol. 336, 1996

104. G. Sundararajan and P.G. Shewmon, The Kinetics of Hydrogen Attack of Steels, Metall. Trans. A, 1981, 12(10), p 1761-1775

105. Y. Shida, T. Kushida, J. Furusawa and H. Fujikawa, Effects of Al and B on Hydrogen Attack of Cr-Mo Steel for Pressure Vessels, in Transactions of the Iron and Steel Institute of Japan, p. B. 26, 1986

106. T. Kushida, J. Furusawa, Y. Shida, T. Kudo and H. Fujikawa, Effect of $\mathrm{Al}, \mathrm{Ti}, \mathrm{B}$ and $\mathrm{N}$ on Hydrogen Attack of Simulated HAZ of Cr-Mo Steel, Tetsu-To-Hagane/J. Iron Steel Inst. Jpn., 1987, 73(14), p 1778 1785

107. Y. Hara, A. Hori and H. Tsukahara, Hydrogen Attack Behavior on Plant Test Specimens, Boshoku gijutsu, 1984, 33(12), p 701-707

108. H.F. Lopez, Effect of Deoxidation Practice and Heat Treatment on the Hydrogen Attack of Carbon Steels, Metall. Trans. A, 1987, 18(11), p 1971-1977
109. T. Su, X. Luo, C. Fan, Y. Li, X. Chen and A. Guo, Tempering Precipitates of Steel 1.25Cr-0.5Mo and Their Effects on Its Hydrogen Attack Resistance, Jinshu Xuebao/Acta Metallurgica Sinica, 1998, 34(4), p 393-399

110. R. Chiba, K. Ohnishi, K. Ishii and K. Maeda, Effect of Heat Treatment on Hydrogen Attack Resistance of C-0.5 Mo Steels for Pressure Vessels, Heat Exchangers, and Piping, Corrosion-NACE, 1985, 41(7), p 415-426

111. R. Chiba, K. Ohnishi, K. Ishii and K. Maeda, Effect of Heat Treatment on Hydrogen Attack Resistance of C-0. 5 Mo Steels for Pressure Vessels, Heat Exchangers, and Piping, Corrosion-NACE, 1985, 41(7), p 415-426

112. R. Chiba, K. Ohnishi, K. Ishii and K. Maeda, Effect of Postweld Heat Treatment on Critical Temperature in Hydrogen Attack of 1/2 Mo Steel Welds, Tetsu-To-Hagane/J. Iron Steel Inst. Jpn., 1987, 73(1), p 175-182

113. M. Prager, Hydrogen Attack Susceptibility of Chrome-Moly Steels and Weldments, in American Society of Mechanical Engineers, Pressure Vessels and Piping Division (Publication) PVP, vol. 239, 1992

114. T. Parthasarathy and P. Shewmon, Hydrogen Attack Behavior of the Heat Affected Zone of a 2.25Cr-1Mo Steel Weldment, Metall. Trans. A, 1987, 18(7), p 1309-1312

115. S.M. Schlögl and E. Van Der Giessen, Micromechanics of High Temperature Hydrogen Attack, Int. J. Numer. Methods Eng., 2001, 52(5-6), p 559-567

116. G. Manna, P. Castello, F. Harskamp, R. Hurst and B. Wilshire, Testing of Welded 2.25CrMo Steel in Hot High-Pressure Hydrogen under Creep Conditions, Eng. Fract. Mech., 2007, 74, p 956-968

117. M. De Marco and G. Mannello, A Novel Approach for Risk Ranking, NDT Priorities and Damage Mitigation of TIP Units Piping Systems in High Temperature Hydrogen Service, 2013

118. Y. Hasegawa, H. Kumagai, F. Kawazoe, M. Okushima, H. Furuya, M. Kodama and T. Nagao, An Evaluation of High Temperature Hydrogen Attack Resistance and a Possible New Microstructure Development Based Remnant Life Assessment Method of a Clean ASME Gr.91 Thick Section Steel Plate, Mater. High Temp., 2017, 34(5-6), p 473481

119. S. Clugston, J. Weertman and P. Shewmon, The Enhancement of Hydrogen Attack in Steel by Prior Deformation, Metall. Trans. A, 1983, 14(4), p 695-699

120. M. Ransick and P. Shewmon, Effect of Cold Work on Hydrogen Attack, Metall. Trans. A, 1981, 12(1), p 17-22

121. F.N. Rhines, The Influence of Gas-Metal Diffusion in Fabricating Processes, American Institute of Mining and Metallurgical Engineers, 1943

122. G.A. Nelson, Metals for High-Pressure Hydrogenation Plants, Trans. ASME, 1951, 73(2), p 205-211

123. G.H. Wagner, A. Heuser and G. Heinke, Hydrogen Attack in 2 1/4 Cr1 Mo Steel Below Nelson's Curve, Ammonia Plant Saf. (Rel. Facil.), 1992, 32, p 252-267

124. D. Eliezer and H.G. Nelson, Hydrogen Attack of 1020 Steel: Influence of Hydrogen Sulfide, Corrosion, 1979, 35(1), p 17-21

125. D.E. Hendrix, Hydrogen Attack on Waterwall Tubes in a High Pressure Boiler, Mater. Perform., 1995, 34(8), p 46-51

126. J. Li and P. Zhang, Steam Line Burst Caused by Hydrogen Attack and Its Prevention, in American Society of Mechanical Engineers, Pressure Vessels and Piping Division (Publication) PVP, vol. 336, 1996

127. B.M. Saba, Evaluation of Mechanical Fitness for Service of High Temperature Hydrogen Attcked Steels, LSU Master's Thesis, 3883, 2003

128. K.L. Baumert, G.V. Krishna and D.P. Bucci, Hydrogen Attack of Carbon-0.5 Molybdenum Piping in Ammonia Synthesis, Mater. Perform., 1986, 25(7), p 34-37

129. N. Ohtsuka and Y. Shindoh, Ultrasonic Monitoring of Hydrogen Attack of $2.25 \mathrm{Cr}-1 \mathrm{Mo}$ Steel, in American Society of Mechanical Engineers, Pressure Vessels and Piping Division (Publication) PVP, vol. 239, pp. 139-143, 1992

130. T. Nomura and T. Imanaka, Ultrasonic Detection of Hydrogen Attack in Low Alloy Steel, in American Society of Mechanical Engineers, Pressure Vessels and Piping Division (Publication) PVP, vol. 276, 1994 
131. K. Kawano, K. Hattori, H. Yamamoto, F. Sakota, H. Okada, T. Tahara, H. Tanaka and T. Ishiguro, UT and Metallurgical Evaluations on Hydrogen Attacked C- 1/2 Mo Steel Pressure Vessels, in American Society of Mechanical Engineers, Pressure Vessels and Piping Division (Publication) PVP, vol. 239, pp. 129-138, 1992

132. M.E. McGovern, D.K. Balch and H. Reis, Nondestructive Detection and Assessment of High Temperature Hydrogen Attack Damage in Carbon Steel Pressure Vessels, in Insight: Non-destructive Testing and Condition Monitoring, vol. 59, no. 1, pp. 11-16, 2017

133. J.F. Conway, M.E. McGovern, D.K. Balch and H. Reis, Nondestructive Detection and Assessment of High Temperature Hydrogen Attack Damage in Carbon Steel Pressure Vessels, in Proceedings of the SPIE, vol. 10600,2018

134. M. Nugent, T. Silfies and J. Dobis, A Review of High Temperature Hydrogen Attack (HTHA) Modelling, Prediction, and Non-Intrusive Inspection in Refinery Applications, in Nace Corrosion Conference \& Expo, 2017

135. M. Nugent, T. Silfies, P. Kowalski and N. Sutton, Recent Applications of Evaluations of Equipment in HTHA Service, 2018

136. C.L. Nevé, S. Loyan, L.L. Jeune, S. Mahaut, S. Demonte, D. Chauveau, R. Renaud, M. Tessier, N. Nourrit and A.L. Guellaut, High Temperature Hydrogen Attack: New NDE Advanced Capabilities-Development and Feedback, in ASME 2019 Pressure Vessels and Piping Conference, PVP2019-94001, 2019

137. A.S. Birring, M. Riethmuller and K. Kawano, Ultrasonic Techniques for Detection of High Temperature Hydrogen Attack, Mater. Eval., 2005, 63(2), p 110-115

138. A. Yamani and M. Deriche, Automatic Detection of High Temperature Hydrogen Attack Defects from Ultrasonic A-Scan Signals, in 2007 IEEE Ultrasonics Symposium, 2007

139. J. Takatsubo and S. Yamamoto, Pore Response Function Method for Quantitative Evaluation of Hydrogen Attack in Steels, Nippon Kikai Gakkai Ronbunshu, A Hen/Trans. Jpn. Soc. Mech. Eng., 1991, 57(543), p 2843-2850

140. G. Kallenberg and T. Munsterman, Remaining Life Assessment of Catalytic Reformer Reactors, 2002

141. J. Takatsubo and H. Yokogawa, Acoustic Emission Source Wave Characteristics of Fracture Process in Hydrogen Attacked Steel, Nippon Kikai Gakkai Ronbunshu, A Hen/Trans. Jpn. Soc. Mech. Eng., 1989, 55(509), p 118-123

142. J. Takatsubo and S. Yamamoto, Fracture Mode Changes According to the Progress of Hydrogen Attack in High-Carbon Steel and Its Acoustic Emission Characteristics, Nippon Kikai Gakkai Ronbunshu, A Hen/Trans. Jpn. Soc. Mech. Eng., 1991, 57(533), p 90-97

143. X. Li, C. Dong, M. Li and H. Chen, Effect of Hydrogen Attack on Acoustic Emission Behavior of Low Carbon Steel, J. Univ. Sci. Technol. Beijing Miner. Metall. Mater. (Eng. Ed.), 2002, 9(2), p 130 134

144. G. Manna, S. Pirfo, L. Debarberis, P. Castello and R. Hurst, Hydrogen Attack Characterisation by Magnetic Measurements, Int. J. Appl. Electromagn. Mech., 2004, 19(1-4), p 597-599

145. R. Grimberg, A. Savin and J. Rebello, Electromagnetic Examination of Hydrogen Attack in AISI 4340 Steel, Stud. Appl. Electromagn. Mech., 2014, 38, p 270-277

146. C Lundin and M Bharadwaj, Metallurgical Assessment and Root Cause Determination of HTHA Damage in Phillips 66 Carbon Steel Pipe-to-Flange Welded Components, in Materials Joining Research, University of Tenessee, Knoxville, 2014

147. T.C. Evans, Hydrogen Attack on Carbon Steels, Mech. Eng., 1948, 70(5), p 414-416

148. T. Sakai and H. Kaji, Nucleation and Growth of Bubbles Formed by Hydrogen Attack in Carbon and Low Alloy Steels, Tetsu-To-Hagane/ J. Iron Steel Inst. Jpn., 1978, 64(3), p 430-439

149. M.L. Martin, M. Dadfarnia, S. Orwig, D. Moore and P. Sofronis, A Microstructure-Based Mechanism of Cracking in High Temperature Hydrogen Attack, Acta Materiallia, 2017, 140, p 300-304

150. T. George, E.R. Parker and R.O. Ritchie, Susceptibility to Hydrogen Attack of a Thick-Section 3Cr-1 Mo-1 Ni Pressure-Vessel Steel-Role of Cooling Rate, Mater. Sci. Technol., 1985, 1(3), p 198-208

151. C. Lundin, M. Bharadwaj, J. Bohling and W. Hoskins, Properties and Microstructural Changes Associated with Long Term Service of Pressure Vessel and Piping Steel at Elevated Temperatures and in Hydrogen and Their Detection and Effects on Remaining Life, in WRC Bulletin Current Concepts for Life Assessment of Pressure
Vessels and Piping, vol. 568, Shaker Heights, Welding Research Council Inc., pp. 171-195, 2016

152. I. Masaoka, I. Takase and S. Ikeda, Criteria for Hydrogen Attack of $1 /$ 2 MoSteel and Cr-Mo Steel Wedments at High Temperature and Pressure, in The American Society for Metals, 1982

153. P. Shewmon, C. Richied, M. McKimpson and M. Ransick, Effect of Hydrogen Pressure on Morphology of Hydrogen Attack, Scr. Metall., 1980, 14, p 545-548

154. Y. Houbaert and J. Dilewijns, Identification and Quantification of Hydrogen Attack, Int. J. Pres. Ves. Piping, 1991, 46, p 113-124

155. P.G. Shewmon, Hydrogen Attack of Carbon Steel, Metall. Trans. A, 1976, 7(2), p 279-286

156. M. Djukic, V.S. Zeravcic, G. Bakic, A. Sedmark and B. Rajicic, Hydrogen Damage of Steel: a Case Study and Hydrogen Embrittlement Model, Eng. Fail. Anal., 2015, 58, p 485-498

157. P. Shewmon and Y.-H. Xue, Effect of High-Pressure Hydrogen on Crack Growth in Carbon Steel, Metall. Trans. A, 1991, 22A, p 27032707

158. L.-C. Chen and P. Shewmon, Stress-Assisted Hydrogen Attack Cracking in $2.25 \mathrm{Cr}-1 \mathrm{Mo}$ Steels at Elevated Temperatures, Metall. Mater. Trans. A., 1995, 26(9), p 2317-2327

159. Y. Tomita and H. Yamamoto, Properties Evaluation of $0.5 \mathrm{Mo}$ Steel Damaged by Hydrogen Attack, in American Society of Mechanical Engineers, Pressure Vessels and Piping Division (Publication) PVP, vol. 380,1998

160. K. Hattori, Advanced Hydrogen Attack Tendency Chart as a Life Assessment Strategy for C-0.5Mo Equipment, in American Society of Mechanical Engineers, Pressure Vessels and Piping Division (Publication) PVP, vol. 359, 1997

161. S. Schlog1, J. Svoboda and E. Van der Geissen, Evolution of the Methane Pressure in a Standard 2.25Cr-1Mo Steel druring Hydrogen Attack, Acta Mater, 2001, 49, p 2227-2238

162. H. Tsubakino, J.-I. Kitasaka and K. Yamakawa, Hydrogen Attack of Carbon Steel Produced by an Electrochemical Method at Elevated Temperatures, Zairyo to Kankyo/ Corros. Eng., 1996, 45(9), p 534539

163. H. Tsubakino, J.-I. Kitasaka and K. Yamakawa, Hydrogen Attack and Its Monitoring by an Electrochemical Method, in American Society of Mechanical Engineers, Pressure Vessels and Piping Division (Publication) PVP, vol. 380, 1998

164. R.E. Allen, P.C. Rosenthal and F.H. Vitovec, Creep Rupture Behavior of Mild Steel under Conditions of Hydrogen Attack, in American Petroleum Institute-Proceedings, 1962

165. K. Yokogawa, S. Fukuyama and K. Kudo, Effect of Stress on Hydrogen Attack of Commercial Pure Iron, Nippon Kinzoku Gakkaisi (J. Jpn. Inst. Met.), 1982, 46(10), p 1009-1017

166. S. Ando, Y. Hisaoka, H. Hamada and K. Yamakawa, Ceramic Sensor for Prediction of Hydrogen Attack, ISIJ Int., 1991, 31(2), p 184-188

167. R. Nishimura, K. Toba and K. Yamakawa, The Developement of a Ceramic Sensor for the Prediction of Hydrogen Attack, Corros. Sci., 1996, 38(4), p 611-621

168. Y.I. Archakov, Modern Problems of Protection of Metals from Hydrogen Attack, Sov. Mater. Sci., 1986, 22(3), p 234-239

169. Y. Archakov and I. Grebeshkova, Ability of Clad Steels to Resist Hydrogen Attack, Chem. Pet. Eng., 1966, 6, p 388-395

170. S. Decker, T. Hynes and G. Buchheim, Safe Operation of a High Temperature Hydrogen Attack Affected DHT Reactor, in Corrosion Conference \& Expo, 2009

171. D. Benac and P. McAndrew, Reducing the Risk of High Temperature Hydrogen Attack (HTHA) Failures, J Fail. Anal. Prevent., 2012, 12, p 624-627

172. C.F. Dong, X.G. Li, Z.S. Shen and W.Y. Chu, Study on Crack Healing of Hydrogen Attack in Carbon Steel by Heat Treatment, Corrosion, 2003, 59(5), p 401-406

173. Steels for Hydrogen Service at Elevated Temperatures and Pressures in Petroleum Refineries and Petrochemical Plants, API Punlication 941, 1st edn., Washington, DC: American Petroleum Institute, 1970

174. J. Cantwell, High-Temperature Hydrogen Attack, Mater. Perform., 1994, 33(7), p 58-61

175. G.A. Nelson, Criteria for Selecting Metals Used in Chemical Plants, in Metal Progress, vol. 77, no. 5, pp. 80-88, 134, 164, 166, 172, 174, 1960

176. G.A. Nelson, When to Use Low Alloy Steel for Hydrogen Service, Hydrocarbon Process. Pet. Refiner, 1966, 45(5), p 201-204 
177. K. Kimura, T. Ishiguro, T. Tahara and K. Kawano, Effect of Metallurgical Variations on Hydrogen Attack Resistance in C-0.5Mo Steel, Tetsu-To-Hagane/J. Iron Steel Inst. Jpn., 1994, 80(12), p 926931

178. K. Kimura, Y. Wada, R. Kayano, T. Ishiguro, K. Hattori and K. Kawano, The Roles of Globular M23C6 Carbides in Hydrogen Attack Resistance in C-0.5Mo Steels, Tetsu-To-Hagane/J. Iron Steel Inst. Jpn., 1999, 85(4), p 332-339

179. K. Kimura, T. Isuiguro, R. Kayano, K. Hattori, K. Kawano and H Yamamoto, Field Surveys of Hydrogen Attack in $0.5 \mathrm{Mo}$ Steel Equipment and Subsequently Observed Metallurgical Effect, TetsuTo-Hagane/J. Iron Steel Inst. Jpn., 1999, 85(10), p 735-742

180. J.E. McLaughlin, A Qualitative Risk-Based Assessment Procedure for High Temperature Hydrogen Attack of C-1/2Mo Steel, in American Society of Mechanical Engineers, Pressure Vessels and Piping Division (Publication) PVP, vol. 7, 2007

181. J. Staats and G. Buchheim, A New Practical Method for Prioritizing Equipment in HTHA Service for Inspection and Replacement and the Challenge in Obtaining Process Conditions to be Used in the HTHA Assessment, in Corrosion 2016 Conference \& Expo Paper No. 7233 2016

182. K. Hattori, K. Kimura, H. Yamamoto and H. Okada, Method of Predicting Hydrogen Attack in 0.5Mo Steel Equipment, Tetsu-ToHagane/J. Iron Steel Inst. Jpn., 1999, 85(10), p 743-750

183. F.H. Vitovec, Investigation of Models for Hydrogen Attack of Steel, $J$. Mater. Sci., 1984, 19(8), p 2771-2774

184. M. McKimpson and P.G. Shewmon, Initial Hydrogen Attack Kinetics in a Carbon Steel, Metall. Trans. A, 1981, 12(5), p 825-834

185. P.G. Shewmon, Synergism between Creep Ductility and Grain Boundary Bubbles, Acta Metall., 1987, 35(6), p 1317-1324

186. M.W. van der Burg and E. van der Giessen, Hydrogen Attack in Creeping Polycrystals Due to Cavitation on Grain Boundaries, in Proceedings of the 1994 5th International Conference on the Effect of Hydrogen on the Behavior of Materials, 1996

187. J.M. Brear and J.M. Church, Technical Basis for API Publication RP941 (Nelson Curves), in Third International Conference on Engineering Structural Integrity Assessment, Cambridge, 1996

188. T.-J. Chuang, K.I. Kagawa, J.R. Rice and L.B. Sills, Overview No. 2: Non-equilibrium Models for Diffusive Cavitation of Grain Interfaces, Acta Metall., 1979, 27(3), p 265-284

189. S.M. Schlögl, Y. Van Leeuwen and E. Van Der Giessen, On Methane Generation and Decarburization in Low-Alloy Cr-Mo Steels during Hydrogen Attack, Metall. Mater. Trans. A., 2000, 31(1), p 125-137

190. E. Van der Giessen and S. Schlogl, Hydrogen Attack, in Handbook of Materials Behavior Models, Academic Press, 2001, pp. 856-863

191. S. Schlogl and E. Van Der Giessen, Computational Model for Carbon Diffusion and Methane Formation in a Ferritic Steel during Hydrogen Attack, Scripta Mater., 2002, 46, p 431-436

192. S. Tang, T. Guo and L. Cheng, Modelling Hydrogen Attack Effect on Creep Fracture Toughness, Int. J. Solids Struct., 2011, 48, p 2909 2919

193. J. Johnson, B. Olson, M. Swindeman, M. Carte and J. Browning, High Temperature Hydrogen Attack Life Assessment Modeling and Inspection, in NACE-International Corrosion Conference Series, v 2019-March, Corrosion 2019; Article Number 13326, 2019

194. S.Z. Chavoshi, L.T. Hill, K.E. Bagnoli, R.L. Holloman and K.M. Nikbin, A Combined Fugacity and Multi-axial Ductility Damage
Approach in Predicting High Temperature Hydrogen Attack in a Reactor Inlet Nozzle, in Engineering Failure Analysis, vol. 117, November, p. 104948, 2020

195. K.E. Bagnoli, Z.A. Cater-Cyker, R. Holloman, C.A. Hay, L.T. Hill, S. Chavoshi and K.M. Nikbin, Volumetric Damage Modeling of High Temperature Hydrogen Attack in Steel Using a Continuum Damage Mechanics Approach, in ASME Pressure Vessels \& Piping Conference-PVP2020-21279, 2020

196. M.I. Kaplan, Simple Model of Steel Interaction with Hydrogen Without Consideration of Carbide Phase Decomposition, Phys. Met. Metall., 1986, 62(2), p 10-15

197. H. Miyaji and H. Nakajima, New Method for Estimating of Radius and Density of Bubbles in Hydrogen Attacked Steel, Trans. Iron Steel Inst. Jpn., 1987, 27(9), p 740-745

198. M. Dadfarnia, M.L. Martin, D.E. Moore, S.E. Orwig and P. Sofronis, A model for high temperature hydrogen attack in carbon steels under constrained void growth, Int. J. Fracture, 2019

199. G. Wilkowski, Y. Hioe, E. Kurth, E. Punch, M. Uddin, F. Brust, K. Bagnoli and G. Pioszak, Initial Developments for LBB Application to HTHA Sensitive Non-stress Relieved Carbon Steel Girth Welds in Refinery Plants," in American Society of Mechanical Engineers, Pressure Vessels and Piping Division (Publication) PVP, 1A-201884669, 2018

200. G. Franceschini, T.P. Silfies, M. Nugent and J. Bednarz, Fleet-Wide HTHA Risk Assessment Using Latest Methodologies, in Ammonia Technical Manual, pp. 145-153, 2018

201. P.E. Prueter, R. Jones, J. Hess and J. DeLuca, Managing the Risks Associated with Operating a Hydrotreater Reactor with Possible High-Temperature Hydrogen Attack Damage," in ASME 2019 Pressure Vessels and Piping Conference, PVP2019-9353, 2019

202. Decarburization, in Damage Mechanisms Affecting Fixed Equipment in the Refining Industry-API RP 571, 3rd edn., American Petroleum Institute, 2020, pp. 130-132

203. Hydrogen Embrittlement, in Damage Mechanisms Affecting Fixed Equipment in the Refining Industry-API RE 571, 3rd edn., American Petroleum Institute, 2020, pp. 213-218

204. Creep and Stress Rupture, in Damage Mechanisms Affecting Fixed Equipment in the Refining Industry-API RP 571, 3rd edn., American Petroleum Institute, 2020, pp. 120-125

205. J. Hirth, Nucleation of Void Sheets in Creep and Hydrogen Attack, Res. Mechanica Lett., 1981, 1(1), p 3-5

206. R. Bruscato, Temper Embrittlement and Creep Embrittlement of 2-1/ 4Cr-1Mo Shielded Metal-Arc Weld Deposits, in Welding Research Supplement, pp. 148s-156s, 1970

207. R.D. Pendse and R.O. Ritchie, A Study of Fatigue Crack Propagation In Prior Hydrogen Attacked Pressure Vessel Steels, Metall. Trans. A, 1985, 16(8), p 1491-1501

208. S.-H. Nahm, U.-B. Baek, C.-M. Suh and Y.-S. Pyun, Study on VHCF Fatigue Behaviors and UNSM Effects of Hydrogen Attacked STS 316L, Trans. Korean Soc. Mech. Eng. A, 2017, 41(11), p 1011-1020

Publisher's Note Springer Nature remains neutral with regard to jurisdictional claims in published maps and institutional affiliations. 